\title{
CARLEMAN ESTIMATES AND UNIQUE CONTINUATION FOR SECOND ORDER PARABOLIC EQUATIONS WITH NONSMOOTH COEFFICIENTS
}

\author{
HERBERT KOCH AND DANIEL TATARU
}

\begin{abstract}
In this work we obtain strong unique continuation results for variable coefficient second order parabolic equations. The coefficients in the principal part are assumed to satisfy a Lipschitz condition in $x$ and a Hölder $C^{\frac{1}{3}}$ condition in time. The coefficients in the lower order terms, i.e. the potential and the gradient potential, are allowed to be unbounded and required only to satisfy mixed norm bounds in scale invariant $L_{t}^{p} L_{x}^{q}$ spaces.
\end{abstract}

\section{INTRODUCTION}

The evolution of the understanding of the strong unique continuation problem for second order parabolic equations mirrors and is closely related to the corresponding strong unique continuation problem for second order elliptic equations. Consequently, we begin with a brief overview of the latter problem.

To a second order elliptic operator $\Delta_{g}=\partial_{i} g^{i j} \partial_{j}$ and potentials $V, W$ in $\mathbb{R}^{n}$ we associate the elliptic equation

$$
-\Delta_{g} u=W \nabla u+V u
$$

Given a function $u \in L_{l o c}^{2}\left(\mathbb{R}^{n}\right)$ and $x_{0} \in \mathbb{R}^{n}$ we say that $u$ vanishes of infinite order at $x_{0}$ if there exists $R$ so that for each integer $N$ we have

$$
\int_{B\left(x_{0}, r\right)}|u|^{2} d x \leq c_{N}^{2} r^{2 N}, \quad r<R
$$

The elliptic strong unique continuation property ESUCP has the form

Let $u$ be a solution to (1.1) which vanishes of infinite order at $x_{0}$. Then $u(x)=0$ for $x$ in a neighborhood of $x_{0}$.

ESUCP

ESUCP type results go back to the pioneering work of Carleman [5] in dimension $n=2$, later extended to higher dimension in by Aronszajn and collaborators [3], 4]. Their results apply to Lipschitz metrics $g$ but only mildly unbounded potentials $V$ and $W$. A key ingredient in their approach was to obtain a class of weighted $L^{2}$ estimates which were later called Carleman estimates. The simplest Carleman estimate has the form

$$
\left\||x|^{-\tau} u\right\|_{L^{2}} \lesssim\left\||x|^{2-\tau} \Delta u\right\|_{L^{2}}
$$

The first author was supported in part by the DFG grant KO1307/5-3. The second author was supported in part by the NSF grant DMS-0301122. Part of the work was done while the first author was supported by the Miller Institute for Basic Research in Science. 
and holds uniformly for $\tau$ away from $\pm\left(\frac{n-2}{2}+N\right)$. This restriction is related to the spectrum of the spherical Laplacian.

Adding some extra convexity to the $|x|^{-\tau}$ weight makes the above estimate more robust and allows one to also use it in the variable coefficient case. The role played by the convexity was further clarified and explained by Hörmander [12], [13], who introduced the pseudoconvexity condition for weights as an almost necessary and sufficient condition in order for the Carleman estimates to hold.

The problem becomes more difficult if one seeks to work with unbounded potentials $V$ in or near the scale invariant $L^{\frac{n}{2}}$ space. There the $L^{2}$ Carleman estimates are insufficient. Instead the key breakthrough was achieved in Jerison-Kenig [15], where the $L^{2}$ Carleman estimates are replaced by $L^{p}$ estimates of the form

$$
\left\||x|^{-\tau} u\right\|_{L^{\frac{2 n}{n-2}}} \lesssim\left\||x|^{-\tau} \Delta u\right\|_{L^{\frac{2 n}{n+2}}}
$$

Relevant to the present paper is also the alternative proof of this result which was given by Jerison [14], taking advantage of Sogge's [25] spectral projection bounds for the spherical Laplacian. In the case of operators with smooth variable coefficients $L^{p}$ Carleman estimates were first obtained by Sogge [26], 27.

Working with gradient potentials in the scale invariant space $W \in L^{n}$ introduces an added layer of difficulty. There not even the $L^{p}$ Carleman estimates can hold. Wolff's solution to this in [29] is a weight osculation argument, which allows one to taylor the weight in the Carleman estimate to the solution $u$, producing estimates of the form

$$
\left\|e^{-\tau \phi(x)} u\right\|_{L^{\frac{2 n}{n-2}}}+\left\|e^{-\tau \phi(x)} W \nabla u\right\|_{L^{\frac{2 n}{n+2}}} \lesssim\left\|e^{\tau \phi(x)} \Delta u\right\|_{L^{\frac{2 n}{n+2}}}
$$

where the choice of $\phi$ depends on both $u, W$ and $\tau$.

Finally, the authors's article [17] combines the ideas above into a nearly optimal scale invariant ESUCP result for the elliptic problem, with (i) a Lipschitz metric $g$, (ii) an $L^{\frac{n}{2}}$ potential $V$, and (iii) an almost $L^{n}$ gradient potential $W$. The present paper is the counterpart of [17] for the parabolic strong unique continuation problem.

We consider the second order backwards parabolic operator

$$
P=\partial_{t}+\partial_{k} g^{k l}(t, x) \partial_{l}
$$

in $\mathbb{R} \times \mathbb{R}^{n}$ and potentials $V, W_{1}, W_{2}$. To these we associate the parabolic equation

$$
P u=V u+W_{1} \nabla_{x} u+\nabla_{x}\left(W_{2} u\right)
$$

Given a function $u \in L_{l o c}^{2}$ and $\left(t_{0}, x_{0}\right) \in \mathbb{R} \times \mathbb{R}^{n}$ we say that $u$ vanishes of infinite order at $\left(t_{0}, x_{0}\right)$ if there exists $R$ so that for each integer $N$ we have

$$
\int_{0}^{r^{2}} \int_{B\left(x_{0}, r\right)}|u|^{2} d x d t \leq c_{N}^{2} r^{2 N}, \quad r<R
$$


Alternatively we may only require that $x \rightarrow u\left(t_{0}, x\right)$ vanishes of infinite order at $x_{0}$, i.e.

$$
\int_{B\left(x_{0}, r\right)}\left|u\left(t_{0}, x\right)\right|^{2} d x \leq c_{N}^{2} r^{2 N}, \quad r<R
$$

The two conditions (1.5) and (1.6) are largely equivalent provided that the coefficients $g^{k l}$ have some uniform regularity as $t \rightarrow t_{0}$. However, our assumptions in this article are not strong enough to guarantee this, therefore we consider the two separate cases.

Now we can define the strong unique continuation property $\operatorname{SUCP}(\mathbf{I})$ :

Let $u$ be a solution to (1.4) which vanishes of infinite order at $\left(t_{0}, x_{0}\right)$. Then $u\left(t_{0}, x\right)=0$ for $x$ in a neighborhood of $x_{0}$.

$\operatorname{SUCP}(\mathbf{I})$

and the slightly stronger variant

Let $u$ be a solution to (1.4) so that $x \rightarrow u\left(t_{0}, x\right)$ vanishes of infinite order at $x_{0}$. Then $u\left(t_{0}, x\right)=0$ for $x$ in a neighborhood of $x_{0}$.

$\operatorname{SUCP}(\mathbf{I I})$

The study of unique continuation for parabolic equations began with early work of Mizohata [21] and Yamabe [30], followed by Saut-Scheurer [23]; $L^{p}$ Carleman estimates were first obtained by Sogge [24].

The study of the parabolic strong unique continuation problem began with work of Lin 20] who considered SUCP(II) for the heat equation with $W=0$ and $V$ bounded and time independent. This continued with work of Chen [6] and Poon [22]. Fernández [11], and Escauriaza, Fernández and Vessella [7] considered SUCP(II) under various assumptions on the coefficients and pointwise bounds for $W=0$ and $V$. It is a consequence of Alessandrini and Vessella [1] that SUCP(I) andSUCP(II) are equivalent under weak assumptions on the coefficients, and they derived SUCP(II) in [2] for bounded $W$ and $V$.

The article of Poon 22 contributed to clarifying the correct form of the $L^{2}$ Carleman estimates for the parabolic strong unique continuation problem in Escauriaza and Fernández's work [9]. In the simplest form, these have the form

$$
\left\|t^{-\tau-\frac{1}{2}} e^{-\frac{x^{2}}{8 t}} u\right\|_{L^{2}} \lesssim\left\|t^{-\tau+\frac{1}{2}} e^{-\frac{x^{2}}{8 t}}\left(\partial_{t}+\Delta\right) u\right\|_{L^{2}}
$$

and hold uniformly with respect to $\tau$ away from $(2 n+\mathbb{N}) / 4$. This restriction is connected with the spectral properties of the Hermite operator.

The $L^{p}$ spectral projection bounds for the Hermite operator were independently obtained by Thangavelu [28] and Kharazdhov [16]; see also the simplified proof in the authors's paper [19]. These bounds were essential in the proof of $L^{p}$ Carleman inequalities for the heat operator of Escauriaza [8] and Escauriaza and Vega [10] which yield $\operatorname{SUCP(I)}$ when $g=I_{n}, W=0$ and $V \in L^{1} L^{\infty}+L^{\infty} L^{n / 2}$.

Our aim is to prove that $\mathbf{S U C P}(\mathbf{I})$ respectively, $\mathbf{S U C P}(\mathbf{I I})$ hold under sharp scale invariant assumptions on the metric $g$ and $L^{p}$ conditions on the potentials $V$ and $W_{1}, W_{2}$. The contribution of this work is comparable to [17] for the elliptic problem: We study almost optimal conditions on 
(1) the coefficients $g$

(2) the potential $V$

(3) the gradient potentials $W_{j}$

The combination of rough variable coefficients and $L^{p}$ conditions on the potential seems to be new. Also, to the best of our knowledge this is the first result on unique continuation for parabolic problems under $L^{p}$ conditions on the coefficients of the gradient term.

For simplicity we always assume that $t_{0}=0, x_{0}=0$. For $\operatorname{SUCP}(\mathbf{I})$ it is natura 1 to consider a larger class of operators $P$ which have the form

$$
P=\frac{\partial}{\partial t}+\partial_{k} g^{k l} \partial_{l}+\frac{x_{k}}{t} d^{k l} \partial_{l}+\partial_{l} d^{k l} \frac{x_{k}}{t}+\frac{x_{k}}{t} e^{k l} \frac{x_{l}}{t}
$$

where $\left(g^{k l}\right),\left(d^{k l}\right)$ and $\left(e^{k l}\right)$ are real valued and $\left(g^{k l}\right)$ and $\left(e^{k l}\right)$ are symmetric.

Then simple scale invariant assumptions for the coefficients would be

$$
\|d\|_{L^{\infty}}+\left\|\left(t+x^{2}\right)^{\frac{1}{2}} \partial_{x} d\right\|_{L^{\infty}}+\left\|t \partial_{t} d\right\|_{L^{\infty}} \ll 1
$$

Here and in the sequel $d$ stands for a generic coefficient of the form $g^{k l}-\delta^{k l}$, $d^{k l}$ and $e^{k l}$. For $V$ and $W_{1,2}$ we could consider conditions of the form

$$
\begin{gathered}
\|V\|_{L^{1} L^{\infty}+L^{\infty} L^{n / 2}} \ll 1, \\
\left\|W_{1,2}\right\|_{L^{2} L^{\infty}+L^{\infty} L^{n}} \ll 1 .
\end{gathered}
$$

Here and in the sequel we use the notation $L^{p} L^{q}=L_{t}^{p} L_{x}^{q}$.

The situation is however more complex and we may take (1.8) to (1.10) only as guidelines. We will have to strengthen (1.8) to include some dyadic summability. on the other hand we are able to weaken the time differentiability to a $C^{\frac{1}{3}}$ Holder condition on small time scales.

We are also able to slightly weaken (1.9) almost to uniform bounds on dyadic sets. However, we are unable to use mixed norms for $W_{1}$ and $W_{2}$, and we restrict ourselves to a summable $L^{n+2}$ norm in dyadic sets.

To state our assumptions on $g, V, W_{1}$ and $W_{2}$ we consider a double infinite dyadic partition of the space,

$$
\mathbb{R}^{+} \times \mathbb{R}^{n}=\bigcup_{i=-\infty}^{\infty} \bigcup_{j=0}^{\infty} A_{i j}
$$

where

(1.12) $A_{i j}=\left\{(t, x) \in \mathbb{R}^{+} \times \mathbb{R}^{n}\left|e^{-4 i-4} \leq t \leq e^{-4 i}, e^{j} \leq 1+2\right| x \mid t^{-\frac{1}{2}} \leq e^{j+1}\right\}$.

Consider the subset of indices

$$
\mathcal{A}=\{(i, j): j \leq 2 i+2\}
$$

defining a partition of the cylinder

$$
Q=[0,1] \times B(0,1)
$$

\footnotetext{
${ }^{1}$ This becomes clearer later on after a change of coordinates and conjugation with respect to a Gaussian weight
} 
Define

$$
\mathcal{A}(\tau)=\left\{(i, j) \in \mathcal{A}: 4 i \geq \ln \tau+1, j \leq \frac{1}{2} \ln \tau+2\right\}
$$

which corresponds to a partition of the cut parabola

$$
Q_{\tau}=\left\{(t, x):|x|^{2} \leq \tau t \leq 1\right\} .
$$

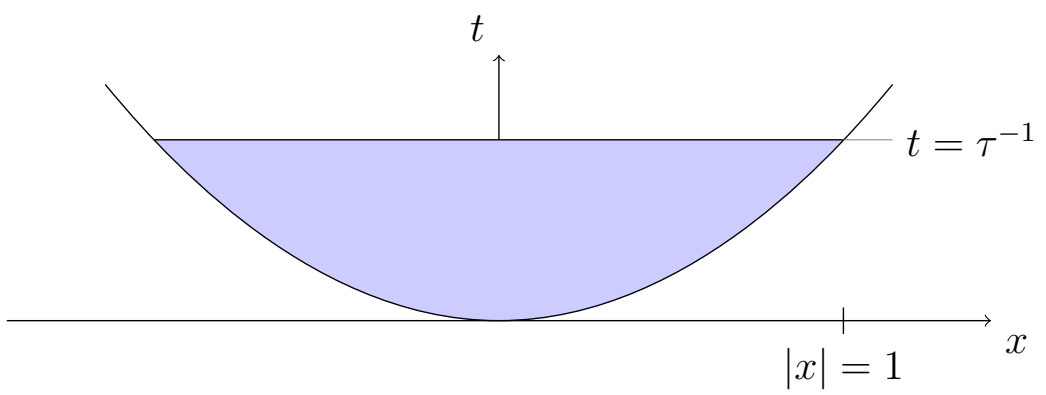

Figure 1. The cut parabola

We also consider a decomposition of $Q$ into dyadic time slices

$$
A_{i}=\left[e^{-4 i-4}, e^{-4 i}\right] \times B(0,1)
$$

and a similar partition of the cut parabola $Q_{\tau}$ into the sets

$$
A_{i}^{\tau}=A_{i} \cap Q_{\tau}
$$

Given a function space $X$ and $1 \leq q<\infty$ we introduce the Banach spaces $l^{q}(\mathcal{A}, X)$ with norms

$$
\|V\|_{l^{q}(\mathcal{A}, X)}^{q}=\sum_{i, j \in \mathcal{A}}\|V\|_{X\left(A_{i j}\right)}^{q} .
$$

In a similar manner we define the spaces $l^{\infty}(\mathcal{A}, X)$.

Within the sets $A_{i j}$ we define the modulus of continuity $\left(m_{i j}\right)$ in time

$$
m_{i j}(\rho)=e^{4 i} \rho+e^{\frac{2}{3}(2 i-j)} \rho^{\frac{1}{3}}
$$

and denote by $C_{t}^{m_{i j}}$ the space of continuous functions with finite seminorm

$$
\|u\|_{C_{t}^{m_{i j}}}=\sup _{t_{1}, t_{2}, x} \frac{\left|u\left(t_{1}, x\right)-u\left(t_{2}, x\right)\right|}{m^{i j}\left(\left|t_{1}-t_{2}\right|\right)}
$$

For the reader's convenience we note that within $A_{i j}$ we have $e^{4 i} \approx t^{-1}$ and $e^{\frac{2}{3}(2 i-j)} \approx\left(t+|x|^{2}\right)^{-\frac{1}{3}}$.

For the coefficients of the operator $P$ in (1.7) we change the condition (1.8) to

$$
\sup _{\tau} \sum_{\mathcal{A}(\tau)}\|d\|_{L^{\infty}\left(A_{i j}\right)}+e^{j-2 i}\|d\|_{\operatorname{Lip}_{x}\left(A_{i j}\right)}+\|d\|_{C_{t}^{m_{i j}\left(A_{i j}\right)}} \ll 1
$$

where we note that $e^{j-2 i} \approx\left(t+x^{2}\right)^{\frac{1}{2}}$ in $A_{i j}$. 
The pointwise bound for $g-I_{n}$ in (1.15), namely

$$
\sup _{\tau}\left\|g-I_{n}\right\|_{l^{1}\left(\mathcal{A}(\tau), L^{\infty}\right)} \ll 1
$$

is not really needed for our results. It can be always obtained from the other bounds after a change of coordinates. This is discussed in the appendix.

The assertion (1.15) is satisfied for $g \in \operatorname{Lip}_{x} \cap C_{t}^{\frac{1}{3}}$ provided that $g(0,0)=I_{n}$. Indeed by scaling we may assume that the $\operatorname{Lip}_{x} \cap C_{t}^{\frac{1}{3}}$ norm is small therefore it suffices to compute

$$
\left\|\left(t+|x|^{2}\right)^{\frac{1}{3}}\right\|_{l^{1}\left(\mathcal{A}(\tau), L^{\infty}\right)} \leq \sum_{i \geq \ln \tau} \sum_{j \leq \ln (\tau) / 2+2} e^{\frac{1}{3}(j-2 i)} \lesssim 1 .
$$

For the potential $V$ we consider:

$$
\begin{aligned}
\|V\|_{l^{\infty}\left(\mathcal{A}, L^{1} L^{\infty}+L^{\infty} L^{n / 2}\right)} & \ll 1 & & \text { for } n>2 \\
\|V\|_{l^{\infty}\left(\mathcal{A}, L^{1} L^{\infty}+L^{p} L^{p^{\prime}}\right)} & \ll 1 & & \text { for } n>2,1 \leq p<\infty, \\
\|V\|_{l^{\infty}\left(\mathcal{A}, L^{1} L^{\infty}+L^{2} L^{1}\right)} & \ll 1 & & \text { for } n=1
\end{aligned}
$$

where $p^{\prime}$ in the second line is the dual exponent. In addition we require that

$$
\sup _{i \geq 0}\left\|\chi_{i} V\right\|_{L^{1} L^{\infty}+L^{\infty} L^{n / 2}} \ll 1 \quad n>2
$$

with the obvious modifications for $n=1,2$, where $\chi_{i}$ is the characteristic function of the set

$$
\left\{(t, x): e^{-4 i-4} \leq t \leq e^{-4 i}, t^{-1 / 2}|x| \leq i\right\} .
$$

Both (1.17) and (1.18) are fulfilled if $V \in L^{1} L^{\infty}+L^{\infty} L^{n / 2}$ with small norm.

Finally for the gradient potentials $W_{1,2}$ we introduce the summability condition with respect to time slices

$$
\sup _{\tau} \sum_{i}\left\|W_{1,2}\right\|_{L^{n+2}\left(A_{i}^{\tau}\right)} \ll 1 .
$$

As a consequence of this we note the uniform bound

$$
\sup _{i}\left\|W_{1,2}\right\|_{L^{n+2}\left(A_{i}\right)} \ll 1 \text {. }
$$

Now we can state our main results.

Theorem 1. Let $P$ be as in (1.7) with coefficients satisfying (1.15). Assume that the potentials $V$ and $W_{1,2}$ satisfy (1.17), (1.18) and (1.19). Then $\mathbf{S U C P}(\mathbf{I})$ holds at $(0,0)$ for $H^{1}$ functions $u$ satisfying (1.4).

It is part of the conclusion that the trace of $u$ at $t=0$ exists near $x=0$. The assumptions on the operator seem to be too weak to imply existence of a trace in general. More precisely shall prove

$$
\|u(t, .)\|_{L^{2}(B(0,1 / 8))} \lesssim e^{-\frac{\delta}{t}}
$$

for some $\delta>0$. 
The $C^{\frac{1}{3}}$ Hölder regularity in time for the metric $g$ seems so be new, improving the $C^{\frac{1}{2}}$ Hölder regularity in [9]. It is not clear to the authors whether this condition is optimal or not.

$\sim \approx$

We may replace the assumptions by stronger translation invariant assumptions,

$$
\begin{aligned}
&\|g\|_{L_{i p_{x}}}+\|g\|_{C_{t}^{\frac{1}{3}}} \lesssim 1 \\
&\|V\|_{L^{1} L^{\infty}+L^{\infty} L^{n / 2}} \ll 1 \\
& \sum_{i}\left\|W_{1,2}\right\|_{L^{n+2}\left(A_{i}\right)} \lesssim 1
\end{aligned}
$$

Then we also obtain a stronger conclusion.

Theorem 2. Let $P$ be as in (1.3) with coefficients as in (1.21). Assume that the potentials $V$ and $W$ satisfy (1.22) respectively (1.23). Then $\mathbf{S U C P ( I I )}$ holds at $(0,0)$ for $H^{1}$ functions u satisfying (1.4).

We remark that the condition (1.21) is really too strong, and that with some additional work (see Remark 2.3) one can bring it almost to the level of (1.15). Precisely, it suffices to replace (1.15) by

$$
\sup _{\tau} \sum_{\mathcal{A}(\tau)}\|d\|_{L^{\infty}\left(A_{i j}\right)}+e^{j-2 i}\|d\|_{\operatorname{Lip}_{x}\left(A_{i j}\right)}+\|d\|_{C_{t}^{m_{i j}^{2}\left(A_{i j}\right)}} \ll 1 .
$$

where the slightly stronger time continuity modulus $m_{i j}^{2}$ is given by

$$
m_{i j}^{2}(\rho)=e^{4 i-2 j} \rho+e^{\frac{2}{3}(2 i-j)} \rho^{\frac{1}{3}}
$$

However, we cannot keep the additional terms in (1.7), because we need to be able to meaningfully talk about the trace of the solution at time $t=0$.

Both theorems are consequences of quantitative estimates, which also imply weak unique continuation under the assumptions of Theorem 2 ;

Let $u$ be a solution to (1.4) for which $u\left(t_{0},.\right)$ vanishes in

the closure of an open set. Then $\left(u\left(t_{0},.\right)\right.$ vanishes in a neighborhood of the closure.

$\mathrm{UCP}$

If $u$ satisfies the assumptions and vanishes in an open set $U$, then it vanishes in the time slices $t=t_{0}$ in an open neighborhood of the closure of $U_{t_{0}}=\{(x:$ $(t, x) \in U\}$.

Theorems 1 and 2 are nontrivial consequences of a Carleman inequality. To state a first version of the Carleman inequality we introduce an additional family $\mathcal{B}(\tau)$ of sets which is a partition of the cylinder $\left[0, \tau^{-1}\right) \times B(0,1)$, consisting of

$$
\begin{gathered}
A_{i j}, \quad \ln \tau \leq 4 i \leq \tau^{1 / 2}, 0 \leq j \leq \ln \tau / 2+2, \\
{\left[e^{-4 i-4}, e^{-4 i}\right] \times B\left(0, e^{-2 i} \tau^{1 / 2}\right), \quad 4 i>\tau^{\frac{1}{2}},}
\end{gathered}
$$




$$
A_{i j}, \quad \ln \tau \leq 4 i, \quad \ln \tau / 2 \leq j \leq 2 i .
$$

This partition is coarser than the partition of the same cylinder into the sets $A_{i j}$. This is the reason why we need the assumption (1.18). More precisely Assumptions (1.17) and (1.18) imply

$$
\|V\|_{l^{\infty}\left(\mathcal{B}(\tau), L^{1} L^{\infty}+L^{\infty} L^{n / 2}\right)} \ll 1 .
$$

Theorem 3. Let $\tau_{0} \gg 1, \varepsilon>0$ and $P$ as in (1.7) with coefficients satisfying (1.15). Suppose that $W_{1,2}$ satisf $Y$ (1.19) with constants depending on $\varepsilon$ and $\tau_{0}$. Then there exists $C>0$ such that for all $\tau \geq \tau_{0}$ the following is true: Suppose that $v \in L^{2}\left(H^{1}\right)$ is compactly supported in $\left[0,8 \tau^{-1}\right) \times B(0,2)$ and that it vanishes of infinite order near $(0,0)$. Then we can find a function $\phi \in C^{\infty}\left(\left[0,8 \tau^{-1}\right] \times B(0,1) \backslash\{0,0\}\right)$ and $h \in C^{\infty}\left(\mathbb{R}^{+}\right)$which satisfy

$$
\tau \leq h^{\prime} \leq(1+\varepsilon) \tau
$$

$$
\left|\phi(x, t)-\left(h(-\ln t)-\frac{x^{2}}{8 t}\right)\right| \leq \varepsilon\left(\tau+\frac{x^{2}}{t}\right)
$$

such that

$$
\left\|e^{\phi} v\right\|_{\left.l^{2}\left(\mathcal{B}(\tau), L^{\infty} L^{2} \cap L^{2} L^{\frac{2 n}{n-2}}\right)\right)} \leq C\left\|e^{\phi}\left(P+W_{1} \nabla+\nabla W_{2}\right) v\right\|_{l^{2}\left(\mathcal{B}(\tau), L^{1} L^{2}+L^{2} L^{\frac{2 n}{n+2}}\right.},
$$

for $n \geq 3$, respectively

$$
\left\|e^{\phi} v\right\|_{l^{2}\left(\mathcal{B}(\tau), L^{\infty} L^{2} \cap L^{p^{\prime}} L^{q^{\prime}}\right)} \leq C\left\|e^{\phi}\left(P+W_{1} \nabla+\nabla W_{2}\right) v\right\|_{l^{2}\left(\mathcal{B}(\tau), L^{1} L^{2}+L^{p} L^{q}\right)},
$$

for $n=2, \frac{1}{p}+\frac{1}{q}=\frac{1}{2}, 2<p$, and

$$
\left\|e^{\phi} v\right\|_{l^{2}\left(\mathcal{B}(\tau), L^{\infty} L^{2} \cap L^{4} L^{\infty}\right)} \leq C\left\|e^{\phi}\left(P+W_{1} \nabla+\nabla W_{2}\right) v\right\|_{l^{2}\left(\mathcal{B}(\tau), L^{1} L^{\infty}+L^{4 / 3} L^{1}\right)}
$$

for $n=1$.

The statement of the Carleman inequality is involved for several reasons. The weight $t^{-\tau} e^{-|x|^{2} / 8 t}$, which works for the constant coefficient case, has to be modified so that it has more convexity in order to handle variable coefficients, spatial localization and the gradient potential. However the polynomial growth in time (imposed by the assumption of vanishing of infinite order) limits the available amount of convexity; this is the origin of the $l^{1}$ summability in (1.16), (1.20), and to a lesser extend of (1.18).

If $W=0$ then the Carleman inequality holds for a large explicit class of weights $e^{\phi}$. This cannot be possibly true for general gradient potentials. Instead, we are only able to prove that there exists some weight function $\phi$, which now depends on $\tau, u$ and $W$, for which the uniform Carleman inequality holds. This strategy goes back to the seminal work of T. Wolff [29] and has been used by the authors for the elliptic problem [17].

The partition $A_{i j}$ is much finer than the dyadic decomposition in $t$ only, which would correspond to the dyadic decomposition in the elliptic case. We are only able to localize the estimates to the sets $A_{i j}$ if we make the weight function sufficiently convex. We can do this for many $A_{i j}$, but not for all of 
them. The sets (1.26) correspond directly to the assumption (1.18). We need to have control of the $L^{1} L^{\infty}+L^{\infty} L^{n / 2}$ norm of $V$ in sets which are not smaller than those of the partition in (1.25), (1.26) and (1.27).

We have stated Theorem 3 in a simpler form which suffices to derive Theorem 1 and Theorem 2. However the full estimate we prove is stronger in that it also contains precise $L^{2}$ bounds. These are essential for the localization and perturbations techniques we use.

The strategy of the proof is the same as in [17]:

(1) We construct families of pseudoconvex weights and derive $L^{2}$ Carleman inequalities. The convexity of weights determines the space-time localization scales and the admissible size of perturbations.

(2) We enhance the above $L^{2}$ Carleman inequalities to include $L^{p}$ estimates. Due to the $L^{2}$ localization it suffices to do this in small sets. This allows us to use perturbation arguments starting from the case of the heat equation with the weight $t^{-\tau} e^{-|x|^{2} / 8 t}$.

(3) $L^{p}$ estimates for the spectral projections to spherical harmonics imply the $L^{p}$ Carleman inequalities in the elliptic case. Here spectral projection for the Hermite operator play a similar role.

(4) Finally we include Wolff's osculating argument into the scheme in order to handle the gradient potentials. The efficiency of this part depends on the flexibility in the choice of the weight functions.

The complexity of the weights and the $L^{2}$ Carleman estimates comes mainly from the geometry of the classical harmonic oscillator. Orbits are contained in a sphere in $\mathbb{R}^{2 n}$. The projection down in the $x$ space is a ball, where frequency variables have a different behavior in radial and angular directions and near the boundary of the the ball. It turns out that our analytic estimates reflect these features.

\section{Proof of Theorem 1 and 2}

In this section we prove Theorem 1 and 2 assuming Theorem 3 . The relation between Carleman estimates and unique continuation is fairly straightforward in the elliptic case. In the parabolic situation the argument is less direct due to the more complex geometry of the level sets of the weight functions.

It is a standard consequence of a localized energy inequality that for the parabolic equation (1.4) $u(t)$ and its gradient can be controlled by $L^{2}$ norms of $u$.

Proposition 2.1. Let $n \geq 3$ and suppose that $v$ solve the parabolic equation

$$
v_{t}+\partial_{k} a^{k l} \partial_{l} v=W_{1} \nabla v+\nabla\left(W_{2} v\right)+V v
$$

on the space-time cylinder $Q=[0,2] \times B(0,2)$ with $a \in$ Lip uniformly elliptic and

$$
\left\|W_{1,2}\right\|_{L^{n+2}(Q)}+\|V\|_{9} L_{L^{1} L^{\infty}+L^{\infty} L^{n / 2}} \ll 1
$$




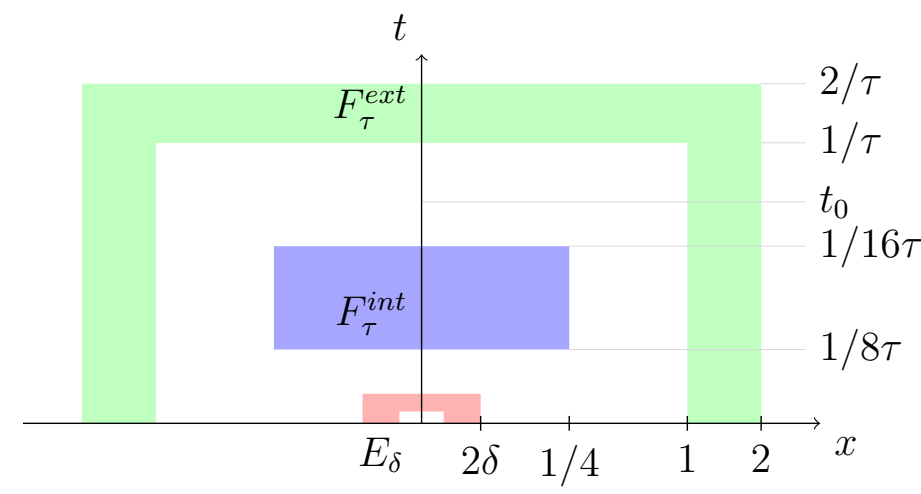

Figure 2. The sets $E_{\delta}, F_{\tau}^{e x t}$ and $F_{\tau}^{\text {int }}$

Then

$$
\sup _{0 \leq t \leq 1}\|v(t)\|_{L^{2}(B(0,1))}+\left\|\nabla_{x} v\right\|_{L^{2}([0,1] \times B(0,1))} \lesssim\|v\|_{L^{2}(Q)} .
$$

If $n=2$ then the same statement is true with $L^{\infty} L^{n / 2}$ replaced by $L^{p} L^{q}$ with $1 \leq p<\infty, 1<q \leq \infty$ and $\frac{1}{p}+\frac{1}{q}=1$. Similarly, if $n=1$ we have to replace it by $L^{4} L^{\infty}$.

Given our assumptions (1.15), (1.17), (1.18), (1.19) and (1.20) we can apply Proposition 2.1 rescaled in sets of the form $\left[t_{0}, 2 t_{0}\right] \times B\left(x_{0}, t_{0}^{1 / 2}\right)$, which are subsets of the $A_{i j}$. Summing up with respect to such sets contained in a parabolic cube

$$
Q_{r}=\left[0, r^{2}\right] \times B(0, r)
$$

we obtain the following consequence.

Corollary 2.2. The following estimate holds under the assumptions of Theorem 1

$$
\left\|t^{\frac{1}{2}} u\right\|_{L^{\infty} L^{2}\left(Q_{r}\right)}+\left\|t^{\frac{1}{2}} \nabla u\right\|_{L^{2}\left(Q_{r}\right)} \lesssim\|u\|_{L^{2}\left(Q_{2 r}\right)}
$$

Proof of Theorem 11. We choose $\tau \geq \tau_{0}$ and $0<\delta \ll \tau^{-1 / 2}$ and introduce the sets

$$
\begin{aligned}
E_{\delta} & =\left(\left[0,2 \delta^{2}\right] \times B(0,2 \delta)\right) \backslash\left(\left[0, \delta^{2}\right] \times B(0, \delta)\right) \\
F_{\tau}^{e x t} & =([0,2 / \tau] \times B(0,2)) \backslash([0,1 / \tau] \times B(0,1)) \\
F_{\tau}^{i n t} & =[1 / 32 \tau, 1 / 16 \tau] \times B(0,1 / 4)
\end{aligned}
$$

Our strategy will be to truncate $u$ in $E_{\delta}$ and $F_{\tau}^{e x t}$ and to apply Theorem 3 to the truncated function in order to obtain a good bound on $u$ in $F_{\tau}^{\text {int }}$.

Let $\eta$ be a cutoff function supported in $[0,2) \times B(0,2)$ and identically 1 in $[0,1] \times B(0,1)$. For $\delta \ll \tau^{-1 / 2}$ we define

$$
v_{\delta}(t, x)=\left(1-\eta\left(t / \delta^{2}, x / \delta\right)\right) \eta(\tau t / 8, x) u(x, t)
$$


which satisfies

$$
(P+W \nabla) v_{\delta}=V v_{\delta}-\left[P+W \nabla, \eta\left(t / \delta^{2}, x / \delta\right)\right] u+[P+W \nabla, \eta(\tau t / 8, x)] u .
$$

The second term on the right hand side is supported in $E_{\delta}$ and the third one in $F_{\tau}^{e x t}$.

We apply Theorem 3 to $v_{\delta}$. One should keep in mind that the corresponding weight $\phi$ depends on $\delta$ but that the bounds we prove are uniform with respect to $\delta$. We normalize the function $h$ by $h(0)=0$. We have to control the size of $\phi$ in the sets $E_{\delta}, F_{\tau}^{\text {ext }}$ and $F_{\tau}^{\text {int }}$. Due to (1.29) we have

$$
\tau s \leq h(s) \leq 2 \tau s, \quad s \geq 0
$$

By (1.30) we obtain a rough polynomial bound in $\delta$

$$
e^{\phi} \leq t^{-2 \tau} e^{-\frac{|x|^{2}}{8 t}+\varepsilon\left(\tau+\frac{x^{2}}{t}\right)} \leq t^{1 / 2} c(\tau) \delta^{-4 \tau-1} \quad \text { in } E_{\delta}
$$

Let

$$
M=\sup \left\{e^{h(-\ln (t))} e^{-\frac{|x|^{2}}{8 t}+\varepsilon\left(\tau+\frac{x^{2}}{t}\right)}:(t, x) \in F_{\tau}^{e x t}\right\}
$$

By (1.29) the supremum is attained at a point $\left(t_{0}, x_{0}\right)$ with $\frac{1}{2} \leq 8 \tau t_{0} \leq 1$ and $\left|x_{0}\right|=1$. A simple computation also shows that

$$
\sup \left\{t^{-1 / 2} e^{h(-\ln (t))} e^{-\frac{|x|^{2}}{8 t}+\varepsilon\left(\tau+\frac{x^{2}}{t}\right)}:(t, x) \in F_{\tau}^{e x t}\right\} \lesssim \tau^{1 / 2} M .
$$

Then $M$ dominates $e^{\phi}$ in $F_{\tau}^{e x t}$ :

$$
e^{\phi} \leq M, \quad t^{-1 / 2} e^{\phi} \lesssim \tau^{1 / 2} M \quad \text { in } F_{\tau}^{e x t} .
$$

Next we need to bound $e^{\phi}$ from below in $F_{\tau}^{\text {int }}$ in terms of $M$,

$$
\inf _{F_{\tau}^{i n t}} e^{\phi} \geq e^{\frac{1}{2} \tau} M
$$

To see this we compute for $(t, x) \in F_{\tau}^{\text {int }}$ and sufficiently small $\varepsilon$ :

$$
\begin{aligned}
\phi(t, x)-\phi\left(t_{0}, x_{0}\right) & \geq h(-\ln t)-h\left(-\ln t_{0}\right)+\frac{1}{8 t_{0}}-\frac{1}{32 t_{0}}-2 \varepsilon\left(\tau+\frac{1}{t_{0}}\right) \\
& \geq\left(\frac{3}{4}-20 \varepsilon\right) \tau \geq \frac{1}{2} \tau
\end{aligned}
$$

and use (2.1) and (2.2).

Theorem 3 applied to $v_{\delta}$ yields

$$
\begin{aligned}
\left\|e^{\phi} v_{\delta}\right\|_{l^{2}\left(\mathcal{B}(\tau), L^{2} L^{\left.\frac{2 n}{n-2} \cap L^{\infty} L^{2}\right)}\right.} \lesssim & \left\|e^{\phi} V v_{\delta}\right\|_{l^{2}\left(\mathcal{B}(\tau), L^{2} L^{\frac{2 n}{n+2}}+L^{1} L^{2}\right)} \\
& +\left\|e^{\phi}\left[P+W \nabla, \eta\left(t / \delta^{2}, x / \delta\right)\right] u\right\|_{l^{2}\left(\mathcal{B}(\tau), L^{2} L^{\frac{2 n}{n+2}}+L^{1} L^{2}\right)} \\
& +\left\|e^{\phi}[P+W \nabla, \eta(\tau t, x)] u\right\|_{l^{2}\left(\mathcal{B}(\tau), L^{2} L^{\frac{2 n}{n+2}}+L^{1} L^{2}\right)}
\end{aligned}
$$

By Hölder's inequality we have

$$
\left\|e^{\phi} V v_{\delta}\right\|_{l^{2}\left(\mathcal{B}(\tau), L^{2} L^{\frac{2 n}{n+2}}+L^{1} L^{2}\right)} \lesssim\|V\|_{l^{\infty}\left(\mathcal{B}(\tau), L^{1} L^{\infty}+L^{\infty} L^{n / 2}\right)}\left\|e^{\phi} v_{\delta}\right\|_{l^{2}\left(\mathcal{B}(\tau), L^{2} L^{\frac{2 n}{n-2}} \cap L^{\infty} L^{2}\right)} .
$$


Due to the smallness in (1.28) we can absorb this term on the left hand side of the inequality.

We calculate the first commutator

$$
\begin{aligned}
f_{\delta} & =\left[P+W \nabla, \eta\left(t / \delta^{2}, x / \delta\right)\right] u \\
& =\left(\frac{\partial}{\partial t}+\partial_{k} g^{k l} \partial_{l}+2 \frac{x_{k}}{t} d^{k l} \partial_{l}+W \nabla\right) \eta\left(t / \delta^{2}, x / \delta\right) \\
& +2 \delta^{-1} g^{k l}\left(\partial_{k} \eta\right)\left(t / \delta^{2}, x / \delta\right) \partial_{l} u
\end{aligned}
$$

By (2.1) we have

$$
\left\|e^{\phi} f_{\delta}\right\|_{l^{2}\left(\mathcal{B}(\tau), L^{2} L^{\frac{2 n}{n+2}}+L^{1} L^{2}\right)} \lesssim c(\tau) \delta^{-4 \tau-1}\left\|t^{\frac{1}{2}} f_{\delta}\right\|_{l^{2}\left(\mathcal{B}(\tau), L^{2} L^{\frac{2 n}{n+2}}+L^{1} L^{2}\right)}
$$

For the $W$ term we use (1.19) and Holder's inequality. For term involving $d^{k l}$ we bound the $L^{1} L^{2}$ norm in terms of an $L^{\infty} L^{2}$ norm, using (1.15) which implies that the pointwise bound for $d^{k l}$ is summable with respect to dyadic time regions. For the remaining terms we simply bound the $L^{1} L^{2}$ norm in terms of the $L^{2}$ norm. This yields

$$
\begin{aligned}
\left\|e^{\phi} f_{\delta}\right\|_{l^{2}\left(\mathcal{B}(\tau), L^{2} L^{\frac{2 n}{n+2}}+L^{1} L^{2}\right)} \lesssim & c(\tau) \delta^{-4 \tau-2}\left(\|u\|_{L^{2}\left(E_{\delta}\right)}+\left\|\left(\partial_{x} \eta\right)\left(t / \delta^{2}, x / \delta\right) t^{\frac{1}{2}} \nabla u\right\|_{L^{2}}\right. \\
& \left.+\left\|\left(\partial_{x} \eta\right)\left(t / \delta^{2}, x / \delta\right) t^{\frac{1}{2}} u\right\|_{L^{\infty} L^{2}}\right)
\end{aligned}
$$

Then we can apply a straightforward modification of Corollary 2.2 on the $E_{\delta}$ scale to finally obtain

$$
\left\|e^{\phi}\left[P+W \nabla, \eta\left(t / \delta^{2}, x / \delta\right)\right] u\right\|_{l^{2}\left(\mathcal{B}(\tau), L^{2} L^{\frac{2 n}{n+2}}+L^{1} L^{2}\right)} \lesssim c(\tau) \delta^{-4 \tau-2}\|u\|_{L^{2}\left(E_{\delta}\right)} .
$$

Similarly we can estimate the second commutator

$$
\left\|e^{\phi}[P+W \nabla, \eta(\tau t, x)] u\right\|_{l^{2}\left(\mathcal{B}(\tau), L^{2} L^{\frac{2 n}{n+2}}+L^{1} L^{2}\right)} \lesssim M \tau^{1 / 2}\|u\|_{L^{2}\left(F_{\tau}^{e x t}\right)}
$$

Hence by inequality (2.4) we get

$$
\left\|e^{\phi} v_{\delta}\right\|_{l^{2}\left(\mathcal{B}(\tau), L^{2} L^{\left.\frac{2 n}{n-2} \cap L^{\infty} L^{2}\right)}\right.} \lesssim M \tau^{1 / 2}\|u\|_{L^{2}\left(F_{\tau}^{e x t}\right)}+c(\tau) \delta^{-4 \tau-2}\|u\|_{L^{2}\left(E_{\delta}\right)} .
$$

Within $F_{\tau}^{\text {int }}$ we have $v_{\delta}=u$. Then by (2.3) we obtain

$$
\|u\|_{L^{\infty} L^{2}\left(F_{\tau}^{\text {int }}\right)} \lesssim \tau^{1 / 2} e^{-\frac{1}{2} \tau}\|u\|_{L^{2}\left(F_{\tau}^{e x t}\right)}+c(\tau) \delta^{-4 \tau-2}\|u\|_{L^{2}\left(E_{\delta}\right)} .
$$

Also by the vanishing of infinite order the second term tends to zero as $\delta \rightarrow 0$. Hence as $\delta \rightarrow 0$ we obtain

$$
\|u\|_{L^{\infty} L^{2}\left(F_{\tau}^{\text {int }}\right)} \lesssim \tau^{1 / 2} e^{-\frac{1}{2} \tau}\|u\|_{L^{2}\left(F_{\tau}^{e x t}\right)}
$$

For $0<t \ll 1$ we choose $\tau=\frac{1}{16 t}$ to obtain

$$
\|u(t, .)\|_{L^{2}(B(0,1 / 4))} \lesssim t^{-1 / 2} e^{-\frac{1}{32 t}} .
$$

This completes the proof of Theorem 1. 
Proof of Theorem 2. We extend the potentials $V$ and $W$ by zero to negative time, and $g^{k l}(t, x)=g^{k l}(0, x)$ for $t<0$. By definition, possibly after rescaling, we have $u(0,.) \in L^{2}(B(0,2))$. We now solve the mixed problem

$$
u_{t}+\partial_{k} g^{k l} \partial_{l} u=0 \quad \text { for } t<0 \text { and }|x| \leq 2
$$

with the boundary condition

$$
u(t, x)=0 \quad \text { if }|x|=2 \text { and } t<0
$$

and the obvious initial condition to obtain an extension of $u$ to negative. The heat kernel for (2.8) satisfies Gaussian estimates. In particular we obtain from (1.6) for all positive integers $N$ with a constant $c_{N}$ possibly differing from (1.6)

$$
\int_{-r^{2}}^{0} \int_{B_{r}(0)}|u|^{2} d x d t \lesssim c_{N}^{2} r^{2 N} .
$$

We seek to prove that the bound (2.7) still holds in this context. The difficulty is that we only know that $u$ vanishes of infinite order at $(0,0)$ for negative time. To account for this we shift the time up, $t \rightarrow t+2 \delta$. Arguing as in the previous proof we obtain (2.6) with $u$ replaced by $u(t+2 \delta, x)$. Letting $\delta \rightarrow 0$ by (2.9) we obtain (2.7) and conclude as above.

Remark 2.3. If one wants to prove Theorem 0 under the weaker assumptions on $g$ in (1.24) then the origin needs to be avoided in the above argument. Hence the time translation needs to be accompanied by a spatial translation, namely

$$
u_{\delta}(t, x)=u\left(t+2 \delta^{2}, x-8 \tau \delta e_{1}\right)
$$

This translation places the image of the origin, or better of the cube $\left[0, \tau \delta^{2}\right] \times$ $B(0,4 \tau \delta)$, within the region $\left\{\tau t<x^{2}\right\}$. But in this region the conjugated operator $P_{\psi}$, introduced later, is elliptic so only pointwise bounds for $g$ are needed for the Carleman estimates.

\section{3. $L^{2}$ BOUNDS IN THE FLAT CASE AND THE HERMite OPERATOR}

In this section we prove the simplest possible $L^{2}$ Carleman estimate for the constant coefficient backward parabolic equation

$$
\partial_{t} u+\Delta_{x} u=f
$$

This serves as a good pretext to introduce the class of weight functions which is later modified for the variable coefficient case.

We also describe the change of coordinates which turns the backward parabolic operator into a forward parabolic equation for the Hermite operator $H$. In this way we are able to relate the $L^{2}$ Carleman estimates for the heat operator to spectral information for $H$. 
Proposition 3.1. Let $u \in L^{2}$ with compact support away from 0 . Then

$$
\left\|t^{-\tau-\frac{1}{2}} e^{-\frac{x^{2}}{8 t}} u\right\|_{L^{2}} \leq\left\|t^{-\tau+\frac{1}{2}} e^{-\frac{x^{2}}{8 t}}\left(\partial_{t}+\Delta\right) u\right\|_{L^{2}}
$$

uniformly with respect to $\tau$ away from $(2 n+\mathbb{N}) / 4$.

Proof. In $\mathbb{R}^{+} \times \mathbb{R}^{n}$ we introduce new coordinates $(s, y) \in \mathbb{R} \times \mathbb{R}^{n}$ defined by

$$
\left\{\begin{array}{l}
t=e^{-4 s} \\
x=\frac{1}{2} e^{-2 s} y
\end{array}\right.
$$

Then

$$
\frac{\partial}{\partial s}=-4 e^{-4 s} \frac{\partial}{\partial t}-e^{-2 s} y \frac{\partial}{\partial x}, \quad \frac{\partial}{\partial y}=\frac{1}{2} e^{-2 s} \frac{\partial}{\partial x}
$$

Hence in the new coordinates our operator becomes

$$
4 t\left(\partial_{t}+\Delta_{x}\right)=-\frac{\partial}{\partial s}-2 y \frac{\partial}{\partial y}+\Delta_{y}
$$

If we conjugate it by $t^{n / 4} e^{-\frac{x^{2}}{8 t}}=e^{-n s} e^{-\frac{y^{2}}{2}}$ we obtain

$$
4 t^{1+n / 4} e^{-\frac{x^{2}}{8 t}}\left(\partial_{t}+\Delta_{x}\right) t^{-n / 4} e^{\frac{x^{2}}{8 t}}=-\frac{\partial}{\partial s}+\Delta_{y}-y^{2}=:-\partial_{s}-H=:-P_{0}
$$

where $H$ is the Hermite operator

$$
H=-\Delta_{y}+y^{2}
$$

Then it is natural to define the new functions

$$
v(s, y)=e^{-n s} e^{-\frac{y^{2}}{2}} u\left(e^{4 s}, \frac{1}{2} e^{2 s} y\right), \quad g(s, y)=e^{(-n-4) s} e^{-\frac{y^{2}}{2}} f\left(e^{4 s}, \frac{1}{2} e^{2 s} y\right)
$$

which are related by

$$
P_{0} v=g \Longleftrightarrow\left(\partial_{t}+\Delta\right) u=f
$$

In the new coordinates, the bound (3.1) becomes

$$
\left\|e^{4 \tau s} v\right\|_{L^{2}} \lesssim\left\|e^{4 \tau s} P_{0} v\right\|_{L^{2}}
$$

Denoting $w=e^{4 \tau s} v$, we conjugate

$$
e^{4 \tau s} P_{0} v=e^{4 \tau s} P_{0} e^{-4 \tau s} w=\left(-\partial_{s}-H+4 \tau\right) w
$$

and the above bound becomes

$$
\|w\|_{L^{2}} \lesssim\left\|\left(-\partial_{s}-H+4 \tau\right) w\right\|_{L^{2}}
$$

Since $\partial_{s}$ and $H-4 \tau$ commute we expand

$$
\left\|\left(-\partial_{s}-H+4 \tau\right) w\right\|_{L^{2}}^{2}=\left\|\partial_{s} w\right\|_{L^{2}}^{2}+\|(H-4 \tau) w\|_{L^{2}}^{2} \geq d(4 \tau, n+\mathbb{N})^{2}\|w\|_{L^{2}}^{2} .
$$

Note the spectral gap, which is essential in order to obtain strong unique continuation results.

For later use we also record the following slight generalization of the above result. For expediency this is stated in the $(y, s)$ coordinates, i.e. in the form of an analogue of (3.3). 
Proposition 3.2. Let $h$ be an increasing, convex, twice differentiable function so that

$$
d\left(h^{\prime}, \mathbb{N}\right)+h^{\prime \prime} \geq \frac{1}{4}
$$

Then

$$
\left\|\left(1+h^{\prime \prime}\right)^{1 / 2} e^{h(s)} v\right\|_{L^{2}}+\left\|\min \left\{1, \frac{\left(1+h^{\prime \prime}\right)^{1 / 2}}{1+h^{\prime}}\right\} e^{h(s)} H v\right\|_{L^{2}} \lesssim\left\|e^{h(s)}\left(\partial_{s}-H\right) v\right\|_{L^{2}}
$$

for all compactly supported $v \in L^{2}$.

Proof. After the substitution $w=e^{h(s)} v$ the bound (3.5) becomes

$$
\left\|\left(1+h^{\prime \prime}\right)^{1 / 2} w\right\|_{L^{2}}^{2}+\left\|\min \left\{1, \frac{\left(1+h^{\prime \prime}\right)^{1 / 2}}{1+h^{\prime}}\right\} H w\right\|_{L^{2}}^{2} \lesssim\left\|\left(\partial_{s}-H+h^{\prime}(s)\right) w\right\|_{L^{2}}^{2}
$$

and we obtain the $L^{2}$ estimate through expanding the term on the right hand side with respect to its selfadjoint and skewadjoint part:

$$
\begin{aligned}
\left\|\left(\partial_{s}-H+h^{\prime}(s)\right) w\right\|_{L^{2}}^{2} & =\left\|\partial_{s} w\right\|_{L^{2}}^{2}+\left\|\left(-H+h^{\prime}\right) w\right\|_{L^{2}}^{2}+\left\|\left(h^{\prime \prime}\right)^{1 / 2} w\right\|_{L^{2}}^{2} \\
& \geq \int\left(d\left(h^{\prime}, \mathbb{N}\right)^{2}+h^{\prime \prime}\right)\|w(s)\|_{L^{2}}^{2} d s
\end{aligned}
$$

To complete the proof we observe that for each $s$ we have

$$
\|H w(s)\|_{L^{2}} \lesssim\left\|\left(-H+h^{\prime}\right) w(s)\right\|_{L^{2}}+h^{\prime}(s)\|w(s)\|_{L^{2}}
$$

\section{Resolvent Bounds For the Hermite operator}

As seen in the previous section, the spectral properties of the Hermite operator play an essential role even in the simplest $L^{2}$ Carleman estimates for the heat equation. In this section we take a look at $L^{2}$ and $L^{p}$ bounds for its spectral projectors and its resolvent.

The spectrum of $H$ is $n+2 \mathbb{N}$, and its eigenfunctions are the Hermite functions defined by

$$
u_{\alpha}=c_{\alpha}\left(\partial_{y}-y\right)^{\alpha} e^{-\frac{x^{2}}{2}}, \quad H u_{\alpha}=(n+2|\alpha|) u_{\alpha}
$$

As $|\alpha|$ increases, so does the multiplicity of the eigenvalues. We denote the spectral projectors by $\Pi_{\lambda}$ for $\lambda \in n+2 \mathbb{N}$. We consider both the spectral projectors and the resolvent of $H$ and obtain both $L^{p}$ and localized $L^{2}$ bounds.

4.1. Weighted $L^{2}$ bounds. We consider two parameters

$$
1 \leq d, R \lesssim \lambda^{\frac{1}{2}}
$$

We denote

$$
B_{R}=\{y:|y|<R\}, \quad, B_{d}^{j}=\left\{y:\left|y_{j}\right|<d\right\}, \quad j=1, \ldots, n
$$


By $\chi_{R}$, respectively $\chi_{d}^{i}$ we denote bump functions in $B_{R}$, respectively $B_{d}^{i}$ which are smooth on the corresponding scales.

Proposition 4.1. The spectral projectors $\Pi_{\lambda}$ satisfy the localized $L^{2}$ bounds

$$
R^{-\frac{1}{2}} \lambda^{\frac{1}{4}}\left\|\chi_{R} \Pi_{\lambda} f\right\|_{L^{2}}+R^{-\frac{1}{2}} \lambda^{-\frac{1}{4}}\left\|\chi_{R} \nabla \Pi_{\lambda} f\right\|_{L^{2}} \lesssim\|f\|_{L^{2}}^{2},
$$

respectively

$$
d^{-\frac{1}{2}}\left\|\left|D_{j}\right|^{\frac{1}{2}}\left(\chi_{d}^{j} \Pi_{\lambda} f\right)\right\|_{L^{2}} \lesssim\|f\|_{L^{2}}
$$

Proof. The inequality (4.1) is trivial unless $R \ll \lambda^{\frac{1}{2}}$. To prove it in dimension $n=1$ we only need to consider the case when $f$ is a Hermite function,

$$
f=\Pi_{\lambda} f=h_{\lambda}
$$

in which case it follows from the pointwise bound

$$
\lambda^{-\frac{1}{4}}\left|h_{\lambda}^{\prime}(x)\right|+\lambda^{\frac{1}{4}}\left|h_{\lambda}(x)\right| \lesssim\left\|h_{\lambda}\right\|_{L^{2}}, \quad|x| \leq \frac{1}{2} \lambda^{\frac{1}{2}}
$$

In dimension $n=1$ (4.2) follows by interpolation from (4.1) with $R=d$. This extends trivially to higher dimension by separation of variables.

It remains to prove (4.1) in higher dimensions. Summing up (4.2) with $d=R$ over $j$ we obtain the bound

$$
R^{-\frac{1}{2}}\left\||D|^{\frac{1}{2}} \chi_{R} \Pi_{\lambda} f\right\|_{L^{2}} \lesssim\|f\|_{L^{2}}^{2}
$$

For $|x| \lesssim R \ll \lambda^{\frac{1}{2}}$ we have $|\xi|^{2} \approx \lambda$ in the characteristic set of $H-\Re z$, therefore the above norm should essentially control the left hand side of (4.1). For later use we prove a slightly more general result, which in particular concludes the proof of (4.1).

$$
\lambda^{\frac{1}{4}}\|v\|_{L^{2}}+\lambda^{-\frac{1}{4}}\|\nabla v\|_{L^{2}} \lesssim\left\||D|^{\frac{1}{2}} v\right\|_{L^{2}}+\left\||y|^{\frac{1}{2}} v\right\|_{L^{2}}+\|(H-\lambda) v\|_{y L^{2}+\nabla L^{2}+\lambda^{\frac{1}{2}} L^{2}}
$$

Indeed the norm on the right is equivalent to

$$
\left\|H^{\frac{1}{4}} v\right\|_{L^{2}}+\left\|(H+\lambda)^{-\frac{1}{2}}(H-\lambda) v\right\|_{L^{2}} \gtrsim \lambda^{\frac{1}{4}}\|v\|_{L^{2}}+\lambda^{-\frac{1}{4}}\left\|H^{\frac{1}{2}} v\right\|_{L^{2}}
$$

In our case we apply (4.3) to $v=R^{-\frac{1}{2}} \chi_{R} \Pi_{\lambda} f$. Then

$$
\left\|\left.y\right|^{\frac{1}{2}} v\right\|_{L^{2}} \lesssim\left\|\Pi_{\lambda} f\right\|_{L^{2}}
$$

while

$$
(H-\lambda) v=2 R^{-\frac{1}{2}} \nabla\left(\nabla \chi_{R} \Pi_{\lambda} f\right)+R^{-\frac{1}{2}} \Delta \chi_{R} \Pi_{\lambda} f
$$

which yields

$$
\|(H-\lambda) v\|_{y L^{2}+\nabla L^{2}+\lambda^{\frac{1}{2}} L^{2}} \lesssim R^{-\frac{3}{2}}\|f\|_{L^{2}}
$$


To state the corresponding resolvent bounds we define the spaces $\tilde{X}_{2}(z)$ by $\|u\|_{\tilde{X}_{2}(z)}=(1+|\Im z|)^{\frac{1}{2}}\|u\|_{L^{2}}+\|(H-z) u\|_{y L^{2}+\nabla L^{2}+|z|^{\frac{1}{2}} L^{2}}+\sup _{j, d} d^{-\frac{1}{2}}\left\|\left|D_{j}\right|^{\frac{1}{2}} \chi_{d}^{j} u\right\|_{L^{2}}$ and the corresponding dual spaces $\tilde{X}_{2}^{*}(z)$. These spaces are larger than the corresponding "elliptic" spaces,

$$
\|v\|_{X_{2}(z)} \lesssim|z|^{\frac{1}{2}}\|v\|_{L^{2}}+\|y v\|_{L^{2}}+\|\nabla v\|_{L^{2}}
$$

On the other hand by extending the bound (4.3) to complex $\lambda$ we obtain a counterpart of (4.1), namely

$$
R^{-\frac{1}{2}} \lambda^{\frac{1}{4}}\left\|\chi_{R} u\right\|_{L^{2}}+R^{-\frac{1}{2}} \lambda^{-\frac{1}{4}}\left\|\chi_{R} \nabla u\right\|_{L^{2}} \lesssim\|u\|_{X_{2}(z)}
$$

Finally, the result of (4.2) can be written in the following dual forms

$$
\left\|\Pi_{\lambda} f\right\|_{X_{2}(\lambda)} \lesssim\|f\|_{L^{2}}, \quad\left\|\Pi_{\lambda} f\right\|_{L^{2}} \lesssim\|f\|_{X_{2}^{*}(\lambda)}
$$

The localized $L^{2}$ resolvent bounds have the form

Proposition 4.2. Let $n \geq 2, z \in \mathbb{C}$ with $\operatorname{dist}(z, n+2 \mathbb{N}) \gtrsim 1$, and $1 \leq d \leq$ $R \ll \Re z$. Then

$$
\|u\|_{\tilde{X}_{2}(z)} \lesssim\|(H-z) f\|_{\tilde{X}_{2}^{*}(z)}
$$

where the $d$ component of norms is omitted in dimension $n=1$.

Proof. We first note that the bounds (4.6) almost imply (4.7) up to a logarithmic divergence. They do imply easily a bound for higher powers of the resolvent for $z$ away from the spectrum of $H$,

$$
\left\|(H-z)^{-1-k} f\right\|_{\tilde{X}_{2}(z)} \lesssim(1+|\Im z|)^{-k}\|f\|_{\tilde{X}_{2}^{*}(z)}, \quad k \geq 1 .
$$

as well as

$$
\|u\|_{L^{2}} \lesssim(1+|\Im z|)^{-\frac{1}{2}}\|(H-z) u\|_{\tilde{X}_{2}^{*}(z)} .
$$

Hence it remains to show that

$$
\|u\|_{\tilde{X}_{2}(z)} \lesssim(1+|\Im z|)^{\frac{1}{2}}\|u\|_{L^{2}}+\|(H-z) u\|_{\tilde{X}_{2}^{*}(z)} .
$$

Using a positive commutator technique we first prove a one dimensional estimate. For this we define the one dimensional skewadjoint pseudodifferential operator

$$
Q_{r}=i O p^{w}\left(\chi\left(y r^{-1}\right) \chi\left(\xi|y|^{-1}\right)\right)
$$

where $\chi$ is a mollified signum function which satisfies

$$
\chi^{\prime}(x)=\frac{1}{4}, \quad|x| \leq 2
$$

Its properties are summarized in the following

\footnotetext{
${ }^{2}$ As defined the symbol of $Q$ is not smooth at 0 . However, any smooth modification in the ball $\left\{x^{2}+\xi^{2}<r^{2}\right\}$ will do.
} 
Lemma 4.3. a) $Q_{r}$ is bounded in $L^{p}$ for $1 \leq p \leq \infty$ uniformly for $r \geq 1$.

b) $Q_{r}$ is also bounded in $\tilde{X}_{2}(z)$ uniformly with respect to $z \in \mathbb{C}$ and $r \geq 1$.

c) $Q_{r}$ satisfies the commutator estimate

$$
r^{-1}\left\||D|^{\frac{1}{2}} \chi_{r} u\right\|_{L^{2}}^{2} \lesssim(1+|\Im z|)\|u\|_{L^{2}}^{2}+\langle(H-z) u, Q u\rangle
$$

Proof. a) The $L^{p}$ boundedness is straightforward and is left for the reader.

b) For the $\tilde{X}_{2}(z)$ boundedness we consider first the $d$ terms, which without any loss in generality we can write in the form

$$
d^{-\frac{1}{2}}\left\|\left(d^{2}+D^{2}\right)^{\frac{1}{4}} \chi_{d} u\right\|_{L^{2}}
$$

Since $Q$ is bounded in $L^{2}$ it suffices to prove the commutator bound

$$
\left\|\left[Q_{r},\left(d^{2}+D^{2}\right)^{\frac{1}{4}} \chi_{d}\right] u\right\|_{L^{2}} \lesssim\|u\|_{L^{2}}
$$

But this is easily verified using the pdo calculus.

Next we consider the term

$$
\|(H-z) u\|_{y L^{2}+\nabla L^{2}+|z|^{\frac{1}{2}} L^{2}}
$$

for which it suffices to prove the commutator bound

$$
\left\|\left[Q_{r}, H\right] u\right\|_{y L^{2}+\nabla L^{2}+|z|^{\frac{1}{2}} L^{2}} \lesssim\|u\|_{L^{2}}
$$

or equivalently, by duality,

$$
\left\|\left[Q_{r}, H\right] u\right\|_{L^{2}} \lesssim\|y u\|_{L^{2}}+\|\nabla u\|_{L^{2}}+\left\||z|^{\frac{1}{2}} u\right\|_{L^{2}}
$$

This follows again from the pseudodifferential calculus.

c) Since $Q_{r}$ is skewadjoint we have the identity

$$
\left\langle(H-z) u, Q_{r} u\right\rangle=\left\langle\left[H, Q_{r}\right] u, u\right\rangle+\Im z\left\langle i Q_{r} u, u\right\rangle
$$

therefore it suffices to insure that

$$
\left\langle\left[H, Q_{r}\right] u, u\right\rangle \gtrsim\|u\|_{\bar{X}_{2}(r)}^{2}+O\left(\|u\|_{L^{2}}^{2}\right) .
$$

For this we compute the commutator $[H, Q]$,

$$
\begin{aligned}
{[H, Q] } & =O p^{w}\left(\left\{\xi_{1}^{2}+y_{1}^{2}, \chi\left(y_{1} r^{-1}\right) \chi\left(\xi_{1}\left|y_{1}\right|^{-1}\right)\right\}\right)+O(1)_{L^{2} \rightarrow L^{2}} \\
& =O p^{w}\left(2 r^{-1} \chi^{\prime}\left(y_{1} r^{-1}\right) \xi_{1} \chi\left(\xi_{1}\left|y_{1}\right|^{-1}\right)\right)+O(1)_{L^{2} \rightarrow L^{2}}
\end{aligned}
$$

Now

$$
r^{-1}\left(\chi_{r}^{1}\right)^{2}\left(\xi_{1}^{2}+r^{2}\right)^{\frac{1}{2}} \lesssim r^{-1} \chi^{\prime}\left(y_{1} r^{-1}\right) \xi_{1} \chi\left(\xi_{1}\left|y_{1}\right|^{-1}\right)+1
$$

and the conclusion follows by Garding's inequality.

We return to the proof of the proposition. By separation of variables the bound (4.11) extends to higher dimensions and gives

$$
r^{-1}\left\|\left|D_{j}\right|^{\frac{1}{2}} \chi_{r}^{j} u\right\|_{L^{2}}^{2} \lesssim(1+|\Im z|)\|u\|_{L^{2}}^{2}+2 \Re\left\langle(H-z) u, Q_{r}^{j} u\right\rangle
$$

where $Q_{r}^{j}$ the higher dimensional analogue of $Q_{r}$ with respect to the $j$ variable. 
The $X_{2}(z)$ boundedness of $Q_{r}$ also extends easily to higher dimension. Hence by Cauchy-Schwartz we obtain

$$
r^{-1}\left\|\left|D_{j}\right|^{\frac{1}{2}} \chi_{r}^{j} u\right\|_{L^{2}}^{2} \lesssim(1+|\Im z|)\|u\|_{L^{2}}^{2}+\|(H-z) u\|_{X_{2}^{*}(z)}\|u\|_{X_{2}(z)}
$$

To conclude the proof of the estimate (4.10) it remains to show that

$$
\|(H-z) u\|_{|z|^{\frac{1}{2}} L^{2}+y L^{2}+\nabla L^{2}} \lesssim\|(H-z) u\|_{X_{2}^{*}(z)}
$$

which follows by duality from (4.4).

The final step in the $L^{2}$ resolvent bounds is to replace the $y^{\prime}$ derivatives by angular derivatives. Let $\nabla_{\perp}=\frac{y}{|y|} \wedge \nabla$ be the angular derivative and $\left|D_{\perp}\right|^{\frac{1}{2}}$ be the corresponding fractional derivative.

We split the coordinates into $y=\left(y_{1}, y^{\prime}\right)$ and use the notation ' for coordinates and derivatives in the obvious sense. For $1 \leq d \leq R \leq \sqrt{\lambda}$ we define the sector

$$
B_{R, d}=\left\{R<\left|y_{1}\right|<2 R, \quad\left|y^{\prime}\right| \leq d\right\}
$$

and $\chi_{R, d}$ a bump function in $B_{R, d}$. Then we define the function space $X_{2}(\lambda, R, d)$ by

$$
\begin{aligned}
\|u\|_{X_{2}(\lambda, R, d)}^{2}= & \|u\|_{L^{2}}^{2}+R^{-1 / 2} \lambda^{-1 / 4}\left\|\nabla\left(\chi_{R, d} u\right)\right\|_{L^{2}}^{2} \\
& +R^{-1 / 2} \lambda^{1 / 4}\left\|\chi_{R, d} u\right\|_{L^{2}}^{2}+d^{-1 / 2}\left\|\left|D_{\perp}\right|^{\frac{1}{2}} \chi_{R, d} u\right\|_{L^{2}}^{2}
\end{aligned}
$$

and $X_{2}^{*}(\lambda, R, d)$ as its dual.

Lemma 4.4. Suppose that $n \geq 2$ and $1 \leq d \leq R$. Then

$$
\|u\|_{X_{2}(\lambda, R, d)} \approx R^{-\frac{1}{2}} \lambda^{\frac{1}{4}}\left\|\chi_{R, d} u\right\|_{L^{2}}+R^{-\frac{1}{2}} \lambda^{-\frac{1}{4}}\left\|\nabla \chi_{R, d} u\right\|_{L^{2}}+d^{-\frac{1}{2}}\left\|\left|D^{\prime}\right|^{\frac{1}{2}} \chi_{R, d} u\right\|_{L^{2}}
$$

Proof. Within $B_{R, d}$ the angular derivatives are close to the $y^{\prime}$ derivatives, namely

$$
\left|D_{\perp} u\right| \lesssim\left|D^{\prime} u\right|+\frac{d}{R}|\nabla u|, \quad\left|D^{\prime} u\right| \lesssim\left|D_{\perp} u\right|+\frac{d}{R}|\nabla u| .
$$

This implies the corresponding bounds for $L^{2}$ norms, and the conclusion follows by interpolation.

¿From the above lemma we obtain

$$
\|u\|_{X_{2}(\lambda, R, d)} \lesssim\|u\|_{\tilde{X}_{2}(\lambda, R, d)}
$$

Hence, we may replace $\tilde{X}_{2}$ by $X_{2}$ in (4.7) and (4.6):

Corollary 4.5. a) For $\lambda$ in the spectrum of $H$ we have

$$
\left\|\Pi_{\lambda} f\right\|_{X_{2}(\lambda, R, d)} \lesssim\|f\|_{L^{2}}, \quad\left\|\Pi_{\lambda} f\right\|_{L^{2}} \lesssim\|f\|_{X_{2}^{*}(\lambda, R, d)}
$$

b) For $z$ away from the spectrum of $H$ and $1 \leq d \leq R \lesssim \Re z$ we have

$$
\left\|(H-z)^{-1-k} f\right\|_{X_{2}(\lambda, R, d)} \lesssim(1+|\Im z|)^{-k}\|f\|_{X_{2}^{*}(\lambda, R, d)}, \quad k \geq 0
$$


4.2. The $L^{p}$ bounds of the resolvent. The $L^{p}$ bounds for the spectral projectors and the resolvent were proved in [16], [28] (see also [10]). For the sake of completeness we also present them here in a simpler manner following the approach in [19]. We refer the reader to the same paper for further results. We consider pairs of exponents satisfying

$$
\frac{2}{p}+\frac{n}{q}=\frac{n}{2}
$$

where the range for $p$ is

$$
p \geq 4 \text { for } n=1, \quad p>2 \text { for } n=2, \quad p \geq 2 \text { for } n \geq 3 .
$$

This leads to the following rang $\epsilon^{3}$ for $q$ :

$$
q \in[2, \infty] \text { for } n=1, \quad q \in[2, \infty) \text { for } n=2, \quad q \in\left[2, \frac{2 n}{n-2}\right] \text { for } n \geq 3 .
$$

The dual exponents are denoted by $p^{\prime}$ and $q^{\prime}$ as usual.

Proposition 4.6. Let $q$ be as in (4.17). Then

a) The spectral projectors $\Pi_{\lambda}$ satisfy

$$
\begin{aligned}
\left\|\Pi_{\lambda}\right\|_{L^{q^{\prime}} \rightarrow L^{2}} \lesssim 1, & \left\|\Pi_{\lambda}\right\|_{L^{2} \rightarrow L^{q}} \lesssim 1, & n \geq 2 \\
\left\|\Pi_{\lambda}\right\|_{L^{q^{\prime}} \rightarrow L^{2}} \lesssim \lambda^{-\frac{1}{p}}, & \left\|\Pi_{\lambda}\right\|_{L^{2} \rightarrow L^{q}} \lesssim \lambda^{-\frac{1}{p}}, & n=1
\end{aligned}
$$

b) For $z$ away from $n+\mathbb{N}$ the resolvent $(H-z)^{-1}$ satisfies

$$
\left\|(H-z)^{-1}\right\|_{L^{q^{\prime}} \rightarrow L^{q}} \lesssim(1+|\Im z|)^{\frac{1}{p}-\frac{1}{p^{\prime}}}, \quad n \geq 1
$$

Outline. To revisit the $L^{p}$ bounds associated to the spectral projectors we recall the approach in [19]. The first step there is to establish pointwise bounds for the Schrödinger evolution 4

$$
\left\|e^{i t H}\right\|_{L^{1} \rightarrow L^{\infty}} \lesssim(\sin t)^{-\frac{n}{2}}
$$

This immediately (see also [18]) leads to Strichartz estimates for the solution to the inhomogeneous equation

$$
i v_{t}-H v=g, \quad v(0)=v_{0}
$$

namely

$$
\|v\|_{L^{p}\left([0,2 \pi] ; L^{q}\right)} \lesssim\left\|v_{0}\right\|_{L^{2}}+\|g\|_{L^{p^{\prime}\left([0,2 \pi] ; L^{q^{\prime}}\right)}}
$$

where $(p, q)$ are as described in (4.15), (4.16).

To obtain (4.18) we apply (4.21) to $v=e^{-i \lambda t} \Pi_{\lambda} u$, which yields $L^{2} \rightarrow L^{p}$ bounds, and hence by duality and selfadjointness all estimates of (4.18) for

\footnotetext{
${ }^{3}$ The exponent $q=\infty$ is actually allowed in the spectral projection bounds in dimension $n=2$. However, it is not allowed in any of the resolvent bounds.

${ }^{4}$ These bounds are very robust and are in effect established in [19] for a much larger class of operators
} 
$n \geq 2$. The case $n=1$ can be dealt with directly using the pointwise bounds for the Hermite functions.

We note a consequence of the bounds (4.18), namely

$$
\left\|(H-z)^{-1-k}\right\|_{L^{q^{\prime}} \rightarrow L^{q}} \lesssim(1+|\Im z|)^{\frac{1}{p}-\frac{1}{p^{\prime}}-k}, \quad n \geq 2, \quad k \geq 1
$$

which is obtained by interpolating between $q=2$ and $q=\frac{2 n}{n-2}$.

Similarly we get

$$
\left\|(H-z)^{-1}\right\|_{L^{q^{\prime}} \rightarrow L^{2}} \lesssim(1+|\Im z|)^{-\frac{1}{p^{\prime}}}, \quad n \geq 2 .
$$

Then we apply (4.21) to

$$
v(x, t)=\chi(t) e^{-i z t} u(x), \quad g=\chi^{\prime}(t) e^{-i z t} u(x)+\chi(t) e^{-i z t}(H-z) u
$$

where $\chi$ is a unit bump function on an interval of size $(1+|\Im z|)^{-1}$. This yields

$$
\|u\|_{L^{q}} \lesssim(1+|\Im z|)^{\frac{1}{p}}\|u\|_{L^{2}}+(1+|\Im z|)^{\frac{1}{p}-\frac{1}{p^{\prime}}}\|(H-z) u\|_{L^{q^{\prime}}} \lesssim\|(H-z) u\|_{L^{q^{\prime}}}
$$

concluding the proof of (4.19) for $n \geq 2$. The case $n=1$ is a variation on the same theme.

4.3. Combining the estimates. Here we combine the $L^{2}$ and the $L^{p}$ components in the resolvent bounds:

Proposition 4.7. For $z$ away from $n+2 \mathbb{N}$ the resolvent $(H-z)^{-1}$ satisfies $(4.24)$

$$
\left\|(H-z)^{-1}\right\|_{L^{q^{\prime}} \rightarrow X_{2}(\Re z, R, d)} \lesssim(1+|\Im z|)^{\frac{1}{p}-\frac{1}{2}}, \quad n \geq 2, \quad(n, q) \neq(2, \infty)
$$

with the obvious modification for $n=1$.

Proof of Proposition 4.7. Taking into account the bounds (4.19) and (4.23), it remains to prove the estimate

$$
\begin{aligned}
\|u\|_{\tilde{X}_{2}(\Re z, R, d)} \lesssim & (1+|\Im z|)^{\frac{1}{2}-\frac{1}{p^{\prime}}}\|(H-z) u\|_{L^{q^{\prime}}} \\
& +(1+|\Im z|)^{\frac{1}{2}-\frac{1}{p}}\|u\|_{L^{q}}+(1+|\Im z|)^{\frac{1}{2}}\|u\|_{L^{2}}
\end{aligned}
$$

But this follows from (4.12) in the same way as for Proposition 4.2 since the operator $Q$ is bounded in $L^{q}$.

\section{5. $L^{p}$ ESTIMATES IN THE FLAT CASE AND PARAMETRIX BOUNDS}

In this section we begin with the mixed norm $L^{p} L^{q}$ Carleman estimates in the simplest case, i.e. with constant coefficients and a polynomial weight. These were proved in [8] except for the endpoint which was obtained later in [10].

After a conformal change of coordinates and conjugation with respect to the exponential weight the Carleman estimates reduce to proving $L^{p} L^{q}$ estimates for a parametrix $K$ for $\partial_{t}-H+\tau$. In this article we need a stronger version of these bounds, where we add in localized $L^{2}$ norms.

In a simplified form, Escauriaza-Vega's result in [10] has the form: 
Theorem 4. [10] Let $p$ and $q$ be as above. Then

$$
\left\|t^{-\tau} e^{-\frac{x^{2}}{8 t} u}\right\|_{L^{\infty}\left(L^{2}\right) \cap L^{p}\left(L^{q}\right)} \leq\left\|t^{-\tau} e^{-\frac{x^{2}}{8 t}}\left(\partial_{t}+\Delta\right) u\right\|_{L^{1}\left(L^{2}\right)+L^{p^{\prime}}\left(L^{q^{\prime}}\right)},
$$

for all $u$ with compact support in $\mathbb{R}^{n} \times[0, \infty)$ vanishing of infinite order at $(0,0)$ uniformly with respect to $4 \tau$ with a positive distance from integers.

One can write the estimate in the $(s, y)$ coordinates using the same transformation as in Section 3 ;

$$
\left\|e^{\tau s} v\right\|_{L^{\infty}\left(L^{2}\right) \cap L^{p}\left(L^{q}\right)} \lesssim\left\|e^{\tau s}\left(\partial_{s}+H\right) v\right\|_{L^{1}\left(L^{2}\right)+L^{p^{\prime}}\left(L^{q^{\prime}}\right)}
$$

Setting $w=e^{\tau s} v$ this becomes

$$
\|w\|_{L^{\infty}\left(L^{2}\right) \cap L^{p}\left(L^{q}\right)} \lesssim\left\|\left(\partial_{s}+H-\tau\right) w\right\|_{L^{1}\left(L^{2}\right)+L^{p^{\prime}}\left(L^{q^{\prime}}\right)}
$$

Denoting by $\Pi_{\lambda}$ the spectral projection onto the $\lambda$ eigenspace of $H$ we obtain a parametrix $K$ for $\left(\partial_{t}-H+\tau\right)$,

$$
K\left(\partial_{t}+H-\tau\right)=I
$$

where the $s$-translation invariant kernel of $K$ is

$$
K(s)=\sum_{\lambda \in \mathbb{N}} \Pi_{\lambda} e^{s(\tau-\lambda)} 1_{s(\tau-\lambda)<0}
$$

Since $w$ decays at $\pm \infty$ we have

$$
w=K\left(\partial_{s}+H-\tau\right) w
$$

therefore (5.2) can be rewritten in the form

$$
\|K f\|_{L^{\infty}\left(L^{2}\right) \cap L^{p}\left(L^{q}\right)} \lesssim\|f\|_{L^{1}\left(L^{2}\right)+L^{p^{\prime}}\left(L^{q^{\prime}}\right)}
$$

The main result of this section is an improvement of (5.2), namely

Proposition 5.1. Assume that $\tau$ is away from $n+\mathbb{N}$ and that

$$
1 \leq d \leq R \lesssim \tau
$$

Then

$$
\|K f\|_{L^{\infty}\left(L^{2}\right) \cap L^{p}\left(L^{q}\right) \cap L^{2} X_{2}(\tau, R, d)} \lesssim\|f\|_{L^{1}\left(L^{2}\right)+L^{p^{\prime}}\left(L^{q^{\prime}}\right)+L^{2} X_{2}^{*}(\tau, R, d)}
$$

Proof. We work in dimension $n \geq 2$; some obvious adjustments are needed in dimension $n=1$, which is slightly easier. We consider four endpoints:

A: The $L^{1} L^{2} \rightarrow L^{\infty} L^{2}$ bound follows easily since the projectors $\Pi_{\lambda}$ are $L^{2}$ bounded.

B: The $L^{1} L^{2} \rightarrow L^{p} L^{q}$ bound. Here it suffices to prove

$$
\|K(.) f\|_{L_{t}^{p} L_{x}^{q}} \lesssim\|f\|_{L^{2}}
$$

Splitting $f$ into spectral projections and using (4.18) we obtain

$$
\|K(t) f\|_{L^{q}} \lesssim \sum_{\lambda} e^{-|(\lambda-\tau) t|}\left\|\Pi_{\lambda} f\right\|_{L^{2}}
$$


For $|t| \geq 1$ we can use Cauchy-Schwartz to obtain

$$
\|K(t) f\|_{L^{q} \cap X_{2}(R, d)} \lesssim e^{-c|t|}\|f\|_{L^{2}}
$$

which suffices for all $q$. For $|t| \leq 1$ we consider the most difficult case $p=2$ and compute

$$
\begin{aligned}
&\|K(t) f\|_{L^{2}\left([-1,1], L^{q}\right)}^{2} \lesssim \int_{-1}^{1}\left(\sum e^{-|(\lambda-\tau) t|}\left\|\Pi_{\lambda} f\right\|_{L^{2}}\right)^{2} \\
&=\sum_{\lambda, \mu} \frac{1}{|\lambda-\tau|+|\mu-\tau|}\left\|\Pi_{\lambda} f\right\|_{L^{2}}\left\|\Pi_{\mu} f\right\|_{L^{2}} \\
& \approx \sum_{0 \leq i, j} 2^{-i-j}\left(\sum_{|\lambda-\tau| \approx 2^{i}}\left\|\Pi_{\lambda} f\right\|_{L^{2}}\right)\left(\sum_{|\mu-\tau| \approx 2^{i+j}}\left\|\Pi_{\mu} f\right\|_{L^{2}}\right) \\
& \lesssim \sum_{0 \leq j} 2^{-\frac{j}{2}} \sum_{0 \leq i}\left(\sum_{|\lambda-\tau| \approx 2^{i}}\left\|\Pi_{\lambda} f\right\|_{L^{2}}^{2}\right)^{\frac{1}{2}}\left(\sum_{|\mu-\tau| \approx 2^{i+j}}\left\|\Pi_{\mu} f\right\|_{L^{2}}^{2}\right)^{\frac{1}{2}} \\
& \lesssim\|f\|_{L^{2}}^{2}
\end{aligned}
$$

C: The $L^{1} L^{2} \rightarrow X_{2}(\tau, R, d)$ bound for $K$ follows in the same way from

$$
\|\Pi u\|_{X_{2}(\tau, R, d)} \lesssim\|u\|_{L^{2}}
$$

D: The $L^{p^{\prime}} L^{q^{\prime}}+L^{2} X_{2}^{*}(\tau, R, d) \rightarrow L^{\infty} L^{2}$ bound for $K$ is equivalent to the $L^{1} L^{2} \rightarrow L^{p} L^{q} \cap X_{2}(\tau, R, d)$ bound for $K^{*}$. By reversing time this is seen to be the same as the $L^{1} L^{2} \rightarrow L^{p} L^{q}$ bound for $K$.

E: The $L^{p^{\prime}} L^{q^{\prime}}+L^{2}\left(X_{2}^{*}(R, d)\right) \rightarrow L^{p} L^{q} \cap L^{2} X_{2}(R, d)$ bound. Using (4.18) and (4.13) directly yields

$$
\|K(s)\|_{L^{\frac{2 n}{n+2}}+X_{2}^{*}(R, d) \rightarrow L^{\frac{2 n}{n-2}} \cap X_{2}(R, d)} \lesssim \sum_{\lambda \in \mathbb{N}} e^{|s(\tau-\lambda)|} \lesssim s^{-1} e^{-c s}
$$

Similarly we obtain

$$
\|K(s)\|_{L^{2} \rightarrow L^{\frac{2 n}{n-2}} \cap X_{2}(R, d)} \lesssim s^{-\frac{1}{2}} e^{-c s}, \quad\|K(s)\|_{L^{\frac{2 n}{n+2}+X_{2}^{*}(R, d)} \rightarrow L^{2}} \lesssim s^{-\frac{1}{2}} e^{-c s}
$$

Interpolation with the $L^{2}$ estimate gives

$$
\|K(s)\|_{L^{q^{\prime}} \rightarrow L^{q}} \lesssim s^{-\frac{2}{p}}
$$

and

$$
\|K(s)\|_{L^{q^{\prime}} \rightarrow X_{2}(R, d)} \lesssim s^{-\frac{1}{p}-\frac{1}{2}}, \quad\|K(s)\|_{X_{2}^{*}(R, d) \rightarrow L^{q}} \lesssim s^{-\frac{1}{p}-\frac{1}{2}}
$$

If $p>2$ then the Hardy-Littlewood Sobolev inequality implies

$$
\|K * f\|_{L^{p} L^{q}} \lesssim\|f\|_{L^{p^{\prime}} L^{q^{\prime}}}
$$

and

$$
\|K * f\|_{L^{2} X_{2}(R, d)} \lesssim\|f\|_{L^{p^{\prime}} L^{q^{\prime}},{ }_{23}}\|K * f\|_{L^{p} L^{q}} \lesssim\|f\|_{L^{2} X_{2}^{*}(R, d)}
$$


With obvious changes the analysis is similar if $n=1,2$.

It remains to prove the $L^{2} \rightarrow L^{2}$ type bounds, namely

$$
\|K f\|_{L^{2} X_{2}(R, d)} \lesssim\|f\|_{L^{2} X_{2}^{*}(R, d)} \quad(n=1,2)
$$

respectively

$$
\|K f\|_{L^{2}\left(X_{2}(R, d) \cap L^{\left.\frac{2 n}{n-2}\right)}\right.} \lesssim\|f\|_{L^{2}\left(X_{2}^{*}(R, d)+L^{\frac{2 n}{n+2}}\right)} \quad(n>2)
$$

For this, following an idea in [10], we consider a dyadic frequency decomposition in time. By the Littlewood-Paley theory it suffices to prove the bound for a single dyadic piece at frequency $2^{j}$, namely

$$
\left\|S_{j}\left(D_{s}\right) K f\right\|_{L^{2}\left(X_{2}(R, d) \cap L^{\frac{2 n}{n-2}}\right)} \lesssim\|f\|_{L^{2}\left(X_{2}^{*}(R, d)+L^{\frac{2 n}{n+2}}\right)} \quad(n>2)
$$

and its one and two dimensional counterpart.

Taking a time Fourier transform we can write (for $f \in \mathcal{S}\left(\mathbb{R}^{n}\right)$ )

$$
\widehat{S_{j} K}(\sigma) f=s\left(2^{-j} \sigma\right) \sum_{\lambda} \frac{1}{\lambda-\tau-i \sigma} \Pi_{\lambda} f=s\left(2^{-j} \sigma\right)(H-\tau-i \sigma)^{-1} f
$$

therefore by the inversion formula

$$
\begin{aligned}
\left(S_{j} K\right)(t) f & =\int e^{i t \sigma} s\left(2^{-j} \sigma\right)(H-\tau-i \sigma)^{-1} f d \sigma \\
& =-t^{-2} \int e^{i t \sigma} \frac{d^{2}}{d \sigma^{2}}\left(s\left(2^{-j} \sigma\right)(H-\tau-i \sigma)^{-1}\right) f d \sigma
\end{aligned}
$$

Hence using the resolvent bounds (4.19) and (4.22) and the first line for $|t| \leq$ $2^{-j}$ and the second line for $|t| \geq 2^{-j}$ we obtain

$$
\left\|S_{j} K(t)\right\|_{L^{\frac{2 n}{n+2}+X_{2}^{*}(R, d)} \rightarrow L^{\frac{2 n}{n-2}} \cap X_{2}(R, d)} \lesssim \frac{2^{j}}{1+2^{2 j} t^{2}}
$$

and the similar estimate in one and two dimensions. The bound on the right is integrable in $t$, therefore (5.5) follows.

\section{MOdIFIED WEIGHTS AND PSEUDOCONVEXITY}

The main result of this section, Theorem 5 is a considerable improvement of Section 3. The weights $t^{-\tau}$ in Section 3, while easy to use, satisfy merely a degenerate pseudoconvexity condition, in the sense that the selfadjoint and the skewadjoint parts of the operator in (3.4) commute. This is in contrast to strong pseudoconvexity where one obtains better $L^{2}$ bounds from the positivity of the commutator. A perturbation argument easily implies an $L^{2}$ Carleman estimate for variable coefficients as soon as $g=I_{n}+O(t)$. However, even arbitrarily small perturbations of $g$ from $I_{n}$ at $t=0$ destroy the pseudoconvexity.

To obtain results for general variable coefficients we need a more robust weight with additional convexity. A good way of doing this is by adding convexity in $t$ and by using a weight of the form

$$
e^{h(-\ln t)}
$$


with a convex function $h$. Then we obtain for the heat operator the strengthened $L^{2}$ estimates of Proposition 3.2 . The assumption of vanishing of infinite order forces us to work with functions $h$ with at most linear growth at infinity. This in turn limits the convexity of $h$, and hence the gain from the convexity in the $L^{2}$ bounds.

These Carleman inequalities with the weight $e^{-h(\ln t)}$ are more stable with respect to perturbations. They can be obtained for coefficients satisfying

$$
\left|g-I_{n}\right|+\left(t+|x|^{2}\right)\left|\partial_{t} g\right|+\left(t+|x|^{2}\right)^{1 / 2}|\nabla g| \lesssim\left(t+|x|^{2}\right)^{\varepsilon} .
$$

with suitable functions $h$. It is not difficult to weaken (6.1) almost to our conditions (1.15) and (1.16). This venue was pursued by Escauriaza and Fernández [9].

In this paper we seek to obtain $L^{p}$ Carleman inequalities and also to handle $L^{p}$ gradient potentials. Both require good spatial and temporal localization, which depends on the strength of the $L^{2}$ estimates. The weights $e^{h(-\ln t)}$ seem to be insufficient for this purpose.

Consequently we consider a larger class of weights of the form

$$
e^{h(-\ln t)+\phi\left(x t^{-1 / 2},-\ln (t)\right)}
$$

having some additional convexity in $y=x t^{-1 / 2}$. Here we think of $\phi$ essentially as a function of $y$ with a milder dependence on $s=-\ln t$. Obtaining pseudoconvexity is not entirely straightforward because the Hamilton flow for the Hermite operator $H$ is periodic so no nonconstant function of $y$ can be convex along its orbits. We note that the projection of the orbits to the $y$ space are ellipses of size $O(\sqrt{\tau})$ where $\tau$ is the energy, centered at 0 . Hence we can choose $\phi$ to be convex in $y$ for $|y| \ll \sqrt{\tau}$. We compensate the lack of convexity of $\phi$ when $|y| \approx \sqrt{\tau}$ by the $s$ convexity of $h$. To elaborate this idea we explain the precise setup.

Let $\delta_{1}$ be small positive constant. We begin with constants $\left\{\alpha_{i j}\right\}_{\mathcal{A}}$ (see (1.13) and (1.14) for the notation) which control the regularity of the coefficient:5 $g^{k l}-\delta^{k l}, d^{k l}$ and $e^{k l}$ of $P$ given by (1.7) as in (1.15).

$$
\delta_{1} \alpha_{i j}=\|d\|_{L^{\infty}\left(A_{i j}\right)}+e^{j-2 i}\|d\|_{\operatorname{Lip}_{x}\left(A_{i j}\right)}+\|d\|_{C_{t}^{m_{i j}\left(A_{i j}\right)}} .
$$

The condition (1.15) guarantee that for all $\tau \geq 1$

$$
\left\|\alpha_{i j}\right\|_{l^{1}(\mathcal{A}(\tau))} \leq 1 . \quad\left\|\alpha_{i j}\right\|_{l^{\infty}(\mathcal{A})} \leq 1
$$

We first adjust the $\alpha_{i j}$ 's upward so that they vary slowly and do not concentrate in irrelevant regions. This readjustment depends on the choice of the parameter $\tau$.

Lemma 6.1. Let $\alpha_{i j}$ be a sequence satisfying (6.3). Then for each $\tau \gg 1$ there exists a double sequence $\left(\varepsilon_{i j}\right)_{\mathcal{A}(\tau)}$ with the following properties:

\footnotetext{
${ }^{5}$ denoted generically by $d$ here and later
} 
(1) For each $(i, j) \in \mathcal{A}(\tau)$

(2) We have $\varepsilon_{i j} \in l^{1}(\mathcal{A}(\tau))$,

$$
\alpha_{i j} \leq \varepsilon_{i j}
$$

$$
\left\|\varepsilon_{i j}\right\|_{l^{1}(\mathcal{A}(\tau))} \lesssim 1
$$

(3) The sequence $\varepsilon_{i j}$ is slowly varying,

$\left|\ln \varepsilon_{i_{1} j_{1}}-\ln \varepsilon_{i_{2} j_{2}}\right| \leq \frac{1}{2}\left(\left|i_{1}-i_{2}\right|+\left|j_{1}-j_{2}\right|\right), \quad\left(i_{1}, j_{1}\right),\left(i_{2}, j_{2}\right) \in \mathcal{A}(\tau)$.

(4) The sequence $\left(\varepsilon_{i}\right)$ defined by

$$
\varepsilon_{i}=\sum_{j:(i, j) \in \mathcal{A}(\tau)} \varepsilon_{i j}, \quad i \geq \ln \tau
$$

has the following properties

$$
\left|\ln \varepsilon_{i_{1}}-\ln \varepsilon_{i_{2}}\right| \leq \frac{1}{2}\left|i_{2}-i_{1}\right|, \quad \varepsilon_{i j} \lesssim \varepsilon_{i}, \quad \varepsilon_{i[\ln \tau / 2]+2} \approx \varepsilon_{i}
$$

(5) For each $i \geq \ln \tau$ there exists an unique $0 \leq j(i) \leq[\ln \tau / 2]+2$ with the following properties:

$$
\varepsilon_{i j(i)} \approx \varepsilon_{i}
$$

and

$$
\begin{array}{ll}
\varepsilon_{i j} \leq e^{-j} \tau^{-1 / 2} & \text { if } 0 \leq j \leq j(i), j(i) \neq 0 \\
\varepsilon_{i j}>e^{-j} \tau^{-1 / 2} & \text { if } j(i)<j \leq[\ln \tau / 2]+2 .
\end{array}
$$

We shall see that $j(i)$ is an important threshold. If $j \geq j(i)$ then we can localize our estimates to the corresponding $A_{i j}$ and even to smaller sets. On the other hand, we cannot localize to sets smaller than

$$
B_{i 0}=\bigcup_{j \leq j(i)} A_{i j}
$$

Proof. To fulfill the conditions (11)-(4) we simply mollify the $\alpha_{i j}$,

$$
\tilde{\varepsilon}_{i j}=\max _{(k, l) \in \mathcal{A}(\tau)} \alpha_{k l} e^{-\frac{1}{2}(|i-k|+|j-l|)}, \quad \tilde{\varepsilon}_{i}=\sum_{j=0}^{[\ln \tau / 2]+2} \tilde{\varepsilon}_{i j} .
$$

For the last part of (44) we redefine

$$
\tilde{\varepsilon}_{i j}:=\tilde{\varepsilon}_{i j}+e^{-\frac{1}{2}|j-([\ln \tau / 2]+2)|} \tilde{\varepsilon}_{i}
$$

This also increases $\tilde{\varepsilon}_{i}$ by a fixed factor.

For (5) we begin with a preliminary guess for $j(i)$ which we call $j_{0}(i) \in \mathbb{R}^{+}$. We consider three cases.

$$
j_{0}(i)=\left\{\begin{array}{ccc}
\ln \tau / 2 & \text { if } & \tilde{\varepsilon}_{i}<\tau^{-1} \\
-\ln \left(\tilde{\varepsilon}_{i} \tau^{1 / 2}\right) & \text { if } & \tau^{-1} \leq \tilde{\varepsilon}_{i}<\tau^{-1 / 2}, \\
0 & \text { if } & \tau^{-1 / 2} \leq \tilde{\varepsilon}_{i} .
\end{array}\right.
$$


We define

$$
\varepsilon_{i j}:=\max \left\{\tilde{\varepsilon}_{i j}, 2 e^{-\frac{1}{2}\left|j-j_{0}(i)\right|} \tilde{\varepsilon}_{i}\right\},
$$

which is still slowly varying because $\tilde{\varepsilon}_{i}$ is slowly varying.

We define $j(i)$ according to (6.6). It is uniquely determined since the sequence $\varepsilon_{i j}$ is slowly varying compared to $e^{-j} \tau^{\frac{1}{2}}$. Since $\tilde{\varepsilon}_{i j}$ is slowly varying we must have $\tilde{\varepsilon}_{i j} \leq \tilde{\varepsilon}_{i} / 2$. This allows us to conclude that for $j$ close to $j_{0}(i)$, the second term in (6.8) is larger than the first one,

$$
\varepsilon_{i j}=2 e^{-\frac{1}{2}\left|j-j_{0}(i)\right|} \tilde{\varepsilon}_{i} \quad \text { for }\left|j-j_{0}(i)\right| \leq 2 .
$$

If $j_{0}(i)=0$ then $\varepsilon_{i 0}=2 \tilde{\varepsilon}_{i} \geq 2 \tau^{-1 / 2}$ and hence $j(i)=0$. If $0<j_{0}(i)<\ln \tau / 2$ then for $\left|j-j_{0}(i)\right| \leq 2$ we have

$$
\varepsilon_{i j}=2 e^{-\frac{1}{2}\left|j-j_{0}(i)\right|} e^{-j_{0}(i)} \tau^{-1 / 2}
$$

therefore $j_{0}(i)-2<j(i) \leq j_{0}(i)$. If $j_{0}(i)=\ln \tau / 2$ then for $\left|j-j_{0}(i)\right| \leq 2$ we have

$$
\varepsilon_{i j} \leq 2 e^{-\frac{1}{2}\left|j-j_{0}(i)\right|} e^{-j_{0}(i)} \tau^{-1 / 2}
$$

and we arrive again at $j_{0}(i)-2 \leq j(i)$. In all three cases we have $\left|j_{0}(i)-j(i)\right| \leq$ 2 therefore (6.5) holds. We observe that $\varepsilon_{i} \lesssim \tilde{\varepsilon}_{i}$.

The sequence $\left(\varepsilon_{i j}\right)_{\mathcal{A}(\tau)}$ is used to describe the amount of spatial convexity needed in the region $A_{i j}$, which will be reflected in the construction of $\phi$ below. The partial sums $\varepsilon_{i}$ measure the amount of $s$-convexity needed in $[i, i+1]$. The purpose of part (5) above is to correlate the two amounts in a region where they have the same strength (where $j$ is close to $j(i)$ ).

Our weights have the form

$$
\psi(s, y)=h(s)+\phi(s, y)
$$

Their choice is described in the next two lemmas:

Lemma 6.2. Let $\tau$ and $\left(\varepsilon_{i}\right)$ be as in Lemma 6.1. Then there is a convex function $h$ with the following properties:

(1) $h^{\prime} \in[\tau, 2 \tau]$.

(2) $h^{\prime \prime}(s)+\operatorname{dist}\left(h^{\prime}(s), \mathbb{N}\right)>\frac{1}{4}$.

(3) $\varepsilon_{i} \tau \lesssim h^{\prime \prime}(s) \lesssim \varepsilon_{i} \tau+1$ for $s \in[i, i+1]$.

(4) $\left|h^{\prime \prime \prime}\right| \lesssim h^{\prime \prime}$.

The proof of the lemma is fairly straightforward and uses only the fact that $\left(\varepsilon_{i}\right)$ is slowly varying and summable. The second part is needed in order to avoid the eigenvalues of the Hermite operator.

Lemma 6.3. Let $\tau,\left(\varepsilon_{i j}\right)$ and $\left(\varepsilon_{i}\right)$ be as in Lemma 6.1. Then there exists a smooth spherically symmetric function

$$
\phi: \mathbb{R} \times \mathbb{R}^{n} \rightarrow \mathbb{R}
$$

with the following properties: 
(1) (Bounds) The function $\phi$ is supported in $|y| \leq 2 \tau^{1 / 2}$ and satisfies

$$
\left.0 \leq \phi(s, y) \lesssim \varepsilon_{i} \tau, \quad \mid \partial_{s} \phi(s, y)\right)|+| \partial_{s}^{2} \phi(s, y) \mid \lesssim \varepsilon_{i} \tau
$$

and

$$
\sum_{l, k=0}^{3}(1+|y|)^{k}\left|D_{y}^{1+k} \partial_{s}^{l} \phi\right| \lesssim \epsilon_{i} \tau^{1 / 2} \quad \text { for } i \leq s \leq i+1,
$$

(2) (Monotonicity)

$$
\partial_{r} \phi(s, y) \approx \varepsilon_{i} \tau^{\frac{1}{2}} \quad \text { for }(s, y) \in A_{i j}, \quad(i, j) \in \mathcal{A}(\tau), \quad j \geq j(i)+1
$$

(3) (Convexity)

$$
(1+|y|) \partial_{r}^{2} \phi(s, y) \approx \varepsilon_{i j} \tau^{\frac{1}{2}} \quad \text { in } A_{i j}, \quad(i, j) \in \mathcal{A}(\tau)
$$

Proof. Let

$$
\phi_{j}(y)=\sqrt{e^{2 j}+|y|^{2}}, \quad j \geq 0 .
$$

We fix a smooth partition of unity $1=\sum \eta(s-i)$ and define

$$
\ln a_{j}(s)=\sum_{i} \eta(s-i) \ln \varepsilon_{i j}
$$

These functions satisfy the bounds

$$
a_{j}(s) \approx \varepsilon_{i j}, \quad i \leq s \leq i+1, \quad\left|a_{j}^{\prime}\right|,\left|a_{j}^{\prime \prime}\right|,\left|a_{j}^{\prime \prime \prime}\right| \lesssim a_{j} .
$$

Their sum satisfies

$$
a(s):=\sum_{j=0}^{[\ln \tau / 2]+2} a_{j}(s) \approx \varepsilon_{i}, \quad i \leq s \leq i+1 .
$$

We define

$$
\phi(s, y)=\tau^{\frac{1}{2}} \chi\left(|y| \tau^{-1 / 2}\right) \sum_{j=0}^{[\ln \tau / 2]+2} a_{j}(s) \phi_{j}(|y|)
$$

where $\chi$ is a smooth function supported in $[-2,2]$ and identically 1 in $\left[-\frac{3}{2}, \frac{3}{2}\right]$. We verify the properties:

$$
0 \leq \phi(s, y) \lesssim a(s) \tau \lesssim \varepsilon_{i} \tau, \quad i \leq s \leq i+1
$$

The remaining part of (6.10) follows from (6.14). Estimate (6.11) is a consequence of (6.14) and

$$
\left|(1+|y|)^{k} D^{k+1} \phi_{j}(y)\right| \lesssim 1, \quad 0 \leq k \leq 3 .
$$

The upper bound from $(6.12)$ is covered by (6.11) and the lower one follows from

$$
\partial_{r} \phi(s, y) \gtrsim \tau^{1 / 2} \sum_{j \leq j(i)} a_{j} \approx \tau^{1 / 2} \sum_{j=0}^{j(i)} \varepsilon_{i j} \sim \varepsilon_{i} \tau^{1 / 2}
$$

in $A_{i j}$ with $j \geq j(i)$ where we use $\varepsilon_{i j(i)} \approx \varepsilon_{i}$. The assertion (6.13) follows from immediate bounds on second derivatives of the $\phi_{j}$. 
Our aim in this section is to prove $L^{2}$ Carleman estimates for the variable coefficient operator $P$ with the exponential weight

$$
e^{\psi\left(-\frac{\ln t}{4}, \frac{2 x}{t^{1 / 2}}\right)} e^{-\frac{|x|^{2}}{8 t}}
$$

Here

$$
\psi(s, y)=h(s)+\delta_{2} \phi(s, y) .
$$

where $\delta_{2}$ is a small constant and $h$ and $\phi$ are as in in Lemma 6.2 and 6.3.

The calculations are involved. For a first orientation we outline the key part of the argument for the constant coefficient heat equation. Using the change of coordinates of Section 3 we transform the problem to weighted estimates for the operator $P_{0}=\partial_{s}+H$ and the exponential weight $e^{\psi(s, y)}$. This translates to obtaining bounds from below for the conjugated operator

$$
P_{0, \psi}=e^{\psi(s, y)} P_{0} e^{-\psi(s, y)} .
$$

Lemma 6.4. Let $\tau$ be large enough. Let $\psi$ be as in (6.9) with $h, \phi$ as in the above two Lemmas 6.2,6.3 with $\delta_{2} \ll 1$. Then the operator $P_{0, \psi}$ satisfies the bound from below

$$
\left\|\left(h^{\prime \prime}\right)^{\frac{1}{2}} v\right\|^{2}+\delta_{2} \tau^{-1}\left(\left\|a_{i n t}^{2} \nabla v\right\|^{2}+\left\|a_{\perp}^{2} \nabla_{\perp} v\right\|^{2}\right) \lesssim\left\|P_{0, \psi} v\right\|_{L^{2}}^{2}
$$

for all functions $v$ supported in $\left\{|y|^{2} \leq 9 \tau\right\}$ where the weights $a_{\text {int }}, a_{\perp}$ are defined by

$$
a_{i n t}^{4}=\varepsilon_{i j}(1+|y|)^{-1} \tau^{\frac{3}{2}}, \quad a_{\perp}^{4}=1_{j \geq j(i)} \varepsilon_{i}(1+|y|)^{-1} \tau^{\frac{3}{2}} \quad \text { in } A_{i j} .
$$

Proof. We decompose $P_{0, \psi}$ into its selfadjoint and its skewadjoint part

$$
P_{0, \psi}=L_{0, \psi}^{r}+L_{0, \psi}^{i}
$$

where

$$
L_{0, \psi}^{r}:=-\Delta_{y}+y^{2}-\psi_{s}-\psi_{y}^{2}, \quad L_{0, \psi}^{i}:=\partial_{s}+\psi_{y} \partial+\partial \psi_{y} .
$$

Expansion of the norm gives

$$
\left\|\left(L_{0 . \psi}^{r}+L_{0, \psi}^{i}\right) v\right\|_{L^{2}}^{2}=\left\|L_{0, \psi}^{r} v\right\|_{L^{2}}^{2}+\left\|L_{0, \psi}^{i} v\right\|_{L^{2}}^{2}+\left\langle\left[L_{0, \psi}^{r}, L_{0, \psi}^{i}\right] v, v\right\rangle
$$

The conclusion of the lemma follows from the commutator bound

$$
\left\langle\left[L_{0, \psi}^{r}, L_{0, \psi}^{i}\right] v, v\right\rangle \gtrsim\left\|\left(h^{\prime \prime}\right)^{\frac{1}{2}} v\right\|^{2}+\delta_{2} \tau^{-1}\left(\left\|a_{i n t}^{2} \nabla v\right\|^{2}+\left\|a_{\perp}^{2} \nabla_{\perp} v\right\|^{2}\right)
$$

The commutator is explicitly computed

$$
\left[L_{0, \psi}^{r}, L_{0, \psi}^{i}\right]=\psi_{s s}+4 \psi_{y} \psi_{y y} \psi_{y}-4 \partial \psi_{y y} \partial-4 y \psi_{y}+4 \psi_{y} \psi_{s y}-\Delta^{2} \psi
$$

Since $\delta_{2} \ll 1$ the first term has size $h^{\prime \prime}(s)$. The second one is nonnegative since $\psi$ is convex for $|y|^{2}<9 \tau$.

The Hessian of the radial function $\psi$ can be written in the form

$$
\psi_{y y}=\psi_{r r} \frac{y}{|y|} \otimes \frac{y}{|y|}+\frac{\psi_{r}}{r}\left(I_{n}-\frac{y}{|y|} \otimes \frac{y}{|y|}\right)
$$


One can see that the radial and angular derivatives carry different weights. Our construction of $\phi$ guarantees that

$$
\psi_{r r} \lesssim \frac{\psi_{r}}{r}, \quad \psi_{y y} \gtrsim \psi_{r r} I_{n}
$$

hence the weight $\psi_{r r}$ can be used for all derivatives. For the size of the two weights we have

$$
\psi_{r r} \approx a_{i n t}^{4}, \quad \frac{\psi_{r}}{r} \gtrsim a_{\perp}^{4}
$$

This gives the last two terms in (6.17).

It remains to see that the remaining terms in the commutator are negligible compared to the first term on the right hand side of (6.17). For this we use the bound (6.11) to conclude that in $A_{i j}$ we have

$$
\left|-4 y \psi_{y}+4 \psi_{y} \psi_{s y}-\Delta^{2} \psi\right| \lesssim \delta_{2} \varepsilon_{i} \tau \lesssim \delta_{2} h^{\prime \prime}
$$

To switch to operators with variable coefficients it is convenient to extend the weights to the full space and to regularize them. Precisely we shall assume that

$$
\begin{array}{ll}
a_{i n t}^{4}(s, y) \approx \varepsilon_{i} \tau & \text { in } A_{i j} \text { if }|y|^{2} \geq \tau \\
a_{i n t}^{4}(s, y) \approx \varepsilon_{i j}(1+|y|)^{-1} \tau^{\frac{3}{2}} & \text { in } A_{i j} \text { if }|y|^{2} \leq \tau .
\end{array}
$$

Observe that the two cases above match since $\varepsilon_{i} \approx \varepsilon_{i j}$ in the region where $y^{2} \approx \tau$. We also introduce a modification $a$ of $a_{\text {int }}$ which is used to include the effect of the spectral gap in regions where we have very little convexity:

$$
\begin{array}{ll}
a^{4}(s, y) \approx 1+\varepsilon_{i} \tau & \text { in } A_{i j} \text { if }|y|^{2} \geq \tau \\
a^{4}(s, y) \approx 1+\varepsilon_{i j}(1+|y|)^{-1} \tau^{\frac{3}{2}} & \text { in } A_{i j} \text { if }|y|^{2} \leq \tau .
\end{array}
$$

Finally we choose $a_{\perp}$ with the properties

$$
\begin{aligned}
& \operatorname{supp} a_{\perp} \subset \bigcup\left\{A_{i j}: j(i)-1 \leq j \leq \frac{1}{2} \ln \tau\right\} \\
& a_{\perp}^{4}(s, y) \lesssim \varepsilon_{i}(1+|y|)^{-1} \tau^{\frac{3}{2}} \quad \text { in } A_{i j} \\
& a_{\perp}^{4}(s, y) \approx \varepsilon_{i}(1+|y|)^{-1} \tau^{\frac{3}{2}} \quad \text { in } A_{i j} \text { if } j(i) \leq j \leq \frac{1}{2} \ln \tau-1
\end{aligned}
$$

The bounds for the weights from above are assumed to remain true after applying powers of the differential operators $\partial_{s}, \partial_{y}$ and $y \partial_{y}$ to them.

Consider now a the more general class of operators $P$ with real variable coefficients given by (1.7). We repeat the change of coordinates and write in the $(s, y)$ coordinates:

$$
4 e^{-4 s} P=-\frac{\partial}{\partial s}-2 y \frac{\partial}{\partial y}+\partial_{i} g^{i j} \partial_{j}+y_{i} d^{i j} \partial_{j}+\partial_{i} d^{i j} y_{j}+y_{i} e^{i j} y_{j}
$$


This further leads to

$$
4 e^{-(n+4) s-\frac{y^{2}}{2}} P e^{n s+\frac{y^{2}}{2}}=-\tilde{P}
$$

where $\tilde{P}$ is given by

$$
\begin{aligned}
\tilde{P}= & \frac{\partial}{\partial s}-\partial_{i} g^{i j} \partial_{j}-y_{i}\left(g^{i j}-2 \delta^{i j}+2 d^{i j}+e^{i j}\right) y_{j} \\
& -y_{i}\left(g^{i j}-\delta^{i j}+d^{i j}\right) \partial_{j}-\partial_{i}\left(g^{i j}-\delta^{i j}\right) y_{j}
\end{aligned}
$$

We rewrite it in the generic form

$$
\tilde{P}=P_{0}-\partial d \partial-y d y-y d \partial-\partial d y
$$

with $P_{0}=\partial_{s}+H$.

To simplify as much as possible the proof of the main $L^{2}$ Carleman estimate we introduce a stronger condition on the regularity of the coefficients:

$$
\begin{aligned}
& |d|+\langle y\rangle\left(\left|d_{y}\right|+\tau^{-\frac{1}{2}}\left|d_{y y}\right|+\tau^{-1}\left|d_{y y y}\right|+\tau^{-\frac{1}{2}}\left|d_{s}\right|\right) \lesssim \delta_{1} \varepsilon_{i j} \quad \text { in } A_{i j} \\
& |d|+\langle y\rangle\left(\left|d_{y}\right|+\tau^{-\frac{1}{2}}\left|d_{y y}\right|+\tau^{-1}\left|d_{y y y}\right|+\tau^{-\frac{1}{2}}\left|d_{s}\right|\right) \lesssim \delta_{1}
\end{aligned}
$$

This improved regularity will be gained later on by regularizing the coefficients. We are now in the position to formulate the Carleman estimate.

Proposition 6.5. Let $\tau$ be large enough and $\delta_{1} \ll \delta_{2} \ll 1$. Let $\psi$ be as in (6.9) with $h, \phi$ as in Lemmas 6.2,6.3. Assume that the coefficients $g-I_{n}, d$ and e satisfy (6.22). Then the following $L^{2}$ Carleman estimate holds for all functions u supported in $\{y \leq 9 \tau\}$ :

$$
\delta_{2}^{\frac{1}{2}}\left(\sum_{j=0,1,2} \tau^{-\frac{j}{2}}\left\|a^{2} e^{\psi} D^{j} u\right\|+\tau^{-\frac{1}{2}}\left\|a_{\perp}^{2} e^{\psi} D_{\perp} u\right\|\right) \lesssim\left\|e^{\psi} \tilde{P} u\right\| .
$$

Proof. After conjugation

$$
P_{\psi}:=e^{\psi(s, y)} \tilde{P} e^{-\psi(s, y)}
$$

we decompose $P_{\psi}$ into its selfadjoint and its skewadjoint part

$$
P_{\psi}=L_{\psi}^{r}+L_{\psi}^{i}
$$

which for $y^{2}<9 \tau$ can be expressed in the generic form (see also (6.15)):

$$
\begin{gathered}
L_{\psi}^{r}=L_{0, \psi}^{r}+\partial d \partial+\tau d \\
L_{\psi}^{i}=L_{0, \psi}^{i}+\tau^{\frac{1}{2}}\left(d \partial_{j}+\partial_{j} d\right)
\end{gathered}
$$

with $d$ satisfying (6.22). Then (6.23) follows from

$$
\sum_{j=0,1,2} \tau^{-j}\left(\delta_{2}\left\|a_{i n t}^{2} D^{j} v\right\|^{2}+\left\langle h^{\prime \prime}\right\rangle^{\frac{1}{2}} D^{j} v \|^{2}\right)+\delta_{2} \tau^{-1}\left\|a_{\perp}^{2} \nabla_{\perp} v\right\|^{2} \lesssim\left\|\tilde{P}_{\psi} v\right\|^{2} .
$$

The proof will consist of three steps. 
Step 1: First we show that for $v$ supported in $\left\{|y|^{2} \leq 9 \tau\right\}$ we have

$$
\frac{\delta_{2}}{\tau}\left(\left\|a_{i n t}^{2} \nabla v\right\|^{2}+\left\|a_{\perp}^{2} \nabla_{\perp} v\right\|^{2}\right)+\left\|\left(h^{\prime \prime}\right)^{\frac{1}{2}} v\right\|^{2}+\left\|L_{\psi}^{r} v\right\|^{2} \lesssim\left\|\tilde{P}_{\psi} v\right\|^{2}+\delta_{1}\left\|a_{i n t}^{2} v\right\|^{2}
$$

We compute

$$
\left\|P_{\psi} v\right\|_{L^{2}}^{2}=\left\|L_{\psi}^{r} v\right\|_{L^{2}}^{2}+\left\|L_{\psi}^{i} v\right\|_{L^{2}}^{2}+\left\langle\left[L_{\psi}^{r}, L_{\psi}^{i}\right] v, v\right\rangle
$$

We expand the commutator

$$
\left[L_{\psi}^{r}, L_{\psi}^{i}\right]=\left[L_{0, \psi}^{r}, L_{0, \psi}^{i}\right]+\left[\partial d \partial+\tau d, L_{0, \psi}^{i}\right]+\tau^{\frac{1}{2}}\left[L_{\psi}^{r}, d \partial_{j}+\partial_{j} d\right]
$$

The main contribution in (6.25) comes from the first commutator, for which we use (6.17) to obtain the terms on the left side of (6.25).

The second commutator is estimated by

$$
\left|\left\langle\left[M^{r}, L_{0, \psi}^{i}\right] v, v\right\rangle\right| \lesssim \delta_{1}\left(\left\|a_{i n t}^{2} v\right\|^{2}+\tau^{-1}\left\|a_{i n t}^{2} \nabla v\right\|^{2}\right)
$$

and the second term on the right is negligible since $\delta_{1} \ll \delta_{2}$. Indeed, we write

$$
\left[\partial d \partial+\tau d, L_{0, \psi}^{i}\right]=-\partial_{k} q^{k l} \partial_{l}+r
$$

where the coefficients $q, r$ have the generic form

$$
q=d_{s}+\psi_{y} d_{y}+\psi_{y y} d+d \psi_{y y}, \quad r=\partial d \partial \Delta \psi+\tau\left(d_{s}+\psi_{y} d_{y}\right)
$$

Using the bounds (6.22) for $d$ and (6.11) for $\phi$ we estimate

$$
|q| \lesssim \delta_{1} \tau^{-1} a_{i n t}^{4}, \quad|r| \lesssim \delta_{1} a_{i n t}^{4}
$$

Finally, the third commutator is estimated in a similar fashion. We write it in the form

$$
\tau^{\frac{1}{2}}\left[L_{\psi}^{r}, d \partial_{j}+\partial_{j} d\right]=-\partial_{k} q^{k l} \partial_{l}+r
$$

where the coefficients $q, r$ have the generic form

$$
q=\tau^{\frac{1}{2}}\left(d_{y}+d d_{y}\right), \quad r=\tau^{\frac{1}{2}}\left(\Delta d_{y}+\partial d \partial d_{y}+d \psi_{y s}+d \psi_{y y} \psi_{y}+\tau d d_{y}\right)
$$

Using (6.22) and (6.11) we obtain the same bounds for $q$ and $r$ as in the previous case. This concludes the proof of (6.25).

Step 2: We use an elliptic estimate to show that for functions $v$ supported in $\left\{|y|^{2} \leq 9 \tau\right\}$ we have

$$
\delta_{2}\left(\sum_{j=0}^{2} \tau^{-j}\left\|a_{i n t}^{2} D^{j} v\right\|^{2}+\tau^{-1}\left\|a_{\perp}^{2} D_{\perp} v\right\|^{2}\right)+\left\|\left(h^{\prime \prime}\right)^{\frac{1}{2}} v\right\|^{2}+\left\|L_{\psi}^{r} v\right\|_{L^{2}}^{2} \lesssim\left\|\tilde{P}_{\psi} v\right\|^{2}
$$

The elliptic bound

$$
\left\|D^{2} v\right\|+\tau\|v\| \lesssim \tau^{\frac{1}{2}}\|D v\|+\left\|\left(-\Delta-h^{\prime}(s)\right) v\right\|
$$

can easily proven by a Fourier transform. It implies

$$
\left\|D^{2} v\right\|+\tau\|v\| \lesssim \tau^{\frac{1}{2}}\|D v\|+\left\|\left(H-h^{\prime}(s)\right) v\right\|+\left\|y^{2} v\right\|,
$$


We can replace $H-h^{\prime}(s)$ by $L_{\psi}^{r}$ due to the pointwise estimate

$$
\left|\left(L_{\psi}^{r}-\left(H-h^{\prime}(s)\right)\right) v\right| \lesssim \delta_{1}\left(\left|D^{2} v\right|+\tau^{\frac{1}{2}}|D v|+\tau|v|\right)
$$

Then (6.26) follows from (6.25).

Step 3: Here we use the spectral gap condition to improve our bound when $h^{\prime \prime} \ll 1$ and show that (6.26) implies (6.23). It suffices to show that if $h^{\prime \prime}(s)<\frac{1}{8}$ then

$$
\|v\|_{L^{2}}+\tau^{-1}\left\|D^{2} v\right\| \lesssim\left\|L_{\psi}^{r} v\right\|_{L^{2}}
$$

Indeed, let $s \in[i, i+1]$ so that $h^{\prime \prime}(s)<\frac{1}{8}$. Then $h^{\prime}$ has a positive distance from the integers. Also $\varepsilon_{i} \lesssim 1$ which implies that at time $s$ we must have

$$
\left|g-I_{n}\right| \lesssim \delta_{1} \tau^{-1}, \quad|D g| \lesssim \delta_{1} \tau^{-1}, \quad\left|\psi_{r}\right| \lesssim \delta_{2} \tau^{-\frac{1}{2}} .
$$

Hence we may think of $L_{\psi}^{r}$ as a small perturbation of $H-h^{\prime}(s)$ and compute

$$
\|v\|+\tau^{-1}\left\|D^{2} v\right\| \lesssim\left\|\left(H-h^{\prime}(s)\right) v\right\| \lesssim\left\|L_{r} v\right\|+\left(\delta_{1}+\delta_{2}^{2}\right)\|v\|+\delta_{1} \tau^{-1}\left\|D^{2} v\right\|
$$

where the last two terms on the right are negligible compared to the left hand side. The proof of the proposition is concluded.

We want to reformulate the previous result in a more symmetric fashion. To do this we weaken the estimates slightly by using a coarser partition of the space. We distinguish three cases for $i$ corresponding to the value of $j(i)$ in Lemma 6.1 (v).

Definition 6.6. We define the partition $B_{i j}$ as follows.

(1) If $j(i)=0$ (which corresponds to $\varepsilon_{i} \gtrsim \tau^{-\frac{1}{2}}$ ) we set

$$
B_{i j}=A_{i j}, \quad b \approx a, b_{\perp} \approx a_{\perp}
$$

(2) If $0<j(i)<[\ln \tau / 2+2]$ (which corresponds to $\tau^{-1} \lesssim \varepsilon_{i} \lesssim \tau^{-\frac{1}{2}}$ ) we set

$$
B_{i j}=A_{i j}, \quad b \approx a, \quad b_{\perp}=a_{\perp} \quad j \geq j(i)
$$

respectively

$$
B_{i 0}=\bigcup_{j<j(i)} A_{i j}, \quad b \approx a_{\mid A_{i j(i)}} \quad b_{\perp}=0 \text { on } B_{i 0}
$$

(3) If $j(i)=[\ln \tau / 2+2]$ (which corresponds to $\tau^{-1} \lesssim \varepsilon_{i}$ ) we set

$$
B_{i 0}=\bigcup_{j=0}^{[\ln \tau / 2]+2} A_{i j}, \quad b=1, \quad b_{\perp}=0 \text { on } B_{i 0} .
$$

Heuristically the definition of the $B_{i j}$ partition is motivated by the fact that in regions $A_{i j}$ with $j<j(i)$ the weight $\phi$ is ineffective, i.e. it changes by at most $O(1)$. Thus the convexity there is useless, and instead we rely directly on localized bounds for the Hermite operator. 
Since the $\varepsilon_{i j}$ are slowly varying $b \lesssim a$ and $b_{\perp} \lesssim a_{\perp}$ and we may replace the $a$ 's by $b$ 's in the above proposition.

To provide some bounds on the size of $b$ and $b_{\perp}$ we introduce a function $1 \leq r(s) \leq \tau^{\frac{1}{2}}$ which is smooth and slowly varying on the unit scale in $s$ so that

$$
r(s) \approx e^{j(i)} \quad s \in[i, i+1]
$$

This describes the region where $b$ is tapered off and $b_{\perp}=0$. Precisely, consider two cases corresponding to the three cases above.

(1) If $r(s) \approx 1$ then we have the bounds

$$
\begin{aligned}
M \tau(1+r)^{-\frac{3}{2}} \lesssim b^{4}(r, s) & \lesssim M \tau(1+r)^{-1} \\
b_{\perp}^{4}(r, s) & \lesssim M \tau(1+r)^{-1}
\end{aligned}
$$

where the parameter $M \geq 1$ is defined by $M \approx \varepsilon(s) \tau^{\frac{1}{2}}$.

(23) If $r(s) \gg 1$ then

$$
\begin{aligned}
\tau r(s)^{-\frac{1}{2}}(r(s)+r)^{-\frac{3}{2}} \lesssim b^{4}(r, s) & \lesssim \tau r(s)^{-1}(r(s)+r)^{-1} \\
b_{\perp}^{4}(r, s) & \lesssim \tau r(s)^{-1}(r(s)+r)^{-1}
\end{aligned}
$$

with approximate equality when $r \lesssim r(s)$ and approximate equality on the right when $r=\tau^{\frac{1}{2}}$.

By slightly changing $b$ and $b_{\perp}$ we may and do assume that the functions $b$ and $b_{\perp}$ are smooth with controlled derivatives. Thus $b$ and $b_{\perp}$ are smooth on the unit scale in $s$ and on the dyadic scale in $y$, and their derivatives satisfy the bounds

$$
\left|b_{s}\right|+\left(r_{s}+r\right)\left|b_{r}\right|+\left(r_{s}+r\right)^{2}\left|b_{r r}\right| \lesssim b, \quad r^{2}<9 \tau
$$

and

$$
\left|b_{\perp s}\right|+\left(r_{s}+r\right)\left|b_{\perp r}\right|+\left(r_{s}+r\right)^{2}\left|b_{\perp r r}\right| \lesssim b_{\perp}+b \quad r^{2}<9 \tau
$$

In addition we have

$$
\operatorname{supp} b_{\perp r} \subset\left\{r>r_{s}\right\}
$$

Using the functions $b$ and $b_{\perp}$ we define the Banach space $X_{2}^{0}$ with norm

$$
\|v\|_{X_{2}^{0}}^{2}=\|b v\|_{L^{2}}^{2}+\tau^{-1 / 2}\left\|b_{\perp} D_{\perp}^{\frac{1}{2}} v\right\|_{L^{2}}^{2}
$$

Then the symmetrized version of Proposition 6.5 has the form

Proposition 6.7. Assume that the coefficients of $P$ satisfy (6.22). Let $\psi$ be as in (6.9) with $h, \phi$ as in Lemmas 6.2,6.3. Then the following $L^{2}$ Carleman estimate holds for all functions u supported in $\{y \leq 9 \tau\}$ :

$$
\left\|e^{\psi(s, y)} u\right\|_{X_{2}^{0}} \lesssim\left\|e^{\psi(s, y)} P u\right\|_{\left(X_{2}^{0}\right)^{*}}
$$


Proof. Conjugating with respect to the exponential weight, the bound (6.32) is rewritten in the form

$$
\|v\|_{X_{2}^{0}} \lesssim\left\|P_{\psi} v\right\|_{\left(X_{2}^{0}\right)^{*}}
$$

Observing that

$$
D_{\perp}^{2}=-y^{-2} \Delta_{\mathbb{S}^{n-1}}
$$

we introduce the operator

$$
Q=Q\left(|y|,\left(-\Delta_{\mathbb{S}^{n-1}}\right)^{\frac{1}{2}}\right), \quad q(r, \lambda)=\left(b^{4}(r)+r^{-2} \tau^{-1} b_{\perp}^{4}(r) \lambda^{2}\right)^{\frac{1}{4}}
$$

Then the inequality (6.33) can be written as

$$
\|Q v\|_{L^{2}} \lesssim\left\|Q^{-1} P_{\psi} v\right\|_{L^{2}}
$$

whereas inequality (6.23) implies

$$
\left\|Q^{2} w\right\|_{L^{2}} \lesssim\left\|P_{\psi} w\right\|_{L^{2}}
$$

Hence it is natural to apply (6.23) to the function $w=Q^{-1} v$, which solves

$$
P_{\psi} w=Q^{-1} P_{\psi} v+Q^{-1}\left[Q, P_{\psi}\right] w
$$

Thus (6.33) would follow provided that the commutator term is small,

$$
\left\|Q^{-1}\left[Q, P_{\psi}\right] w\right\|_{L^{2}} \ll\left\|b^{2} w\right\|_{L^{2}}+\tau^{-1 / 2}\left\|b^{2} \nabla w\right\|_{L^{2}}+\tau^{-1}\left\|b^{2} D_{y}^{2} w\right\|_{L^{2}}
$$

Unfortunately a direct computation shows that the smallness fails when $j$ is close to $j(i)$ even in the flat case, i.e. with $P_{\psi}$ replaced by

$$
P_{0, \psi}=\partial_{s}-\Delta+y^{2}-\psi_{s}-\psi_{y}^{2}
$$

To remedy this we introduce an additional small parameter $\delta$ and use it to define a modification $Q_{\delta}$ of $Q$. We modify $r(s)$ to $r_{\delta}(s)$ defined by

$$
r_{\delta}(s)^{-2}=\delta^{8} r(s)^{-2}+\delta^{2} \tau^{-1}
$$

and use it to define the function

$$
b_{\delta}(r, s)^{4}=\delta^{-12} \tau\left(r^{2}+r_{\delta}(s)^{2}\right)^{-1}
$$

We can still compare it with $b$,

$$
b_{\delta}(r, s)^{4} \lesssim \delta^{-4} b(r, s)^{4}
$$

Its usefulness lies in the fact that it is larger than $b$ exactly in the region where the commutator term above is not small.

The modification $Q_{\delta}$ of $Q$ has symbol

$$
q_{\delta}(r, s, \lambda)=q(r, s, \lambda)+b_{\delta}(r, s)=\left(b^{4}(r, s)+r^{-2} \tau^{-1} b_{\perp}^{4}(r, s) \lambda^{2}\right)^{\frac{1}{4}}+b_{\delta}(r, s)
$$

which satisfies

$$
q \leq q_{\delta} \lesssim \delta^{-1} q
$$

We claim that it satisfies the bound

$$
\left\|Q_{\delta}^{-1}\left[Q_{\delta}, P_{\psi}\right] w\right\|_{L^{2}} \lesssim\left(\delta+c(\delta) \delta_{1}\right) \sum_{j=0,1,2} \tau^{-\frac{j}{2}}\left\|b^{2} D^{j} w\right\|_{L^{2}}
$$


Suppose this is true. Then we fix $\delta$ sufficiently small, and for $\delta_{1}$ small enough we apply (6.23) to $w=Q_{\delta}^{-1} v$. By (6.35) he commutator term in the equation for $w$ can be neglected, and we obtain

$$
\left\|Q^{2} w\right\|_{L^{2}} \lesssim\left\|Q_{\delta}^{-1} P_{\psi} v\right\|_{L^{2}}
$$

which by (6.34) implies that

$$
\|Q v\|_{L^{2}} \lesssim \delta^{-1}\left\|Q^{-1} P_{\psi} v\right\|_{L^{2}}
$$

It remains to prove (6.35).

I. We first calculate the commutator in the flat case, i.e. with $P_{\psi}$ replaced by $P_{0, \psi}$. Due to the spherical symmetry the only contribution comes from the radial part of the Laplacian and the $s$ derivative. Hence using polar coordinates we compute

$$
Q_{\delta}^{-1}\left[Q_{\delta}, P_{0, \psi}\right]=Q_{\delta}^{-1}\left(Q_{\delta r r}+\frac{n-1}{r} Q_{\delta r}+2 Q_{\delta r} \partial_{r}-Q_{\delta s}\right)
$$

Then is suffices to verify that on the symbol level we have

$$
\left|q_{\delta r r}\right|+r^{-1}\left|q_{\delta r}\right|+\left|q_{\delta s}\right|+\tau^{1 / 2}\left|q_{\delta r}\right| \lesssim \delta q_{\delta} b^{2}\left(1+\tau^{-1} r^{-2} \lambda^{2}\right)^{\frac{1}{2}}
$$

I.(1). We begin with the $q$ component of $q_{\delta}$. Using (6.29) and (6.30) one obtains

$$
\left|q_{r r}\right|+r^{-1}\left|q_{r}\right|+\left|q_{s}\right|+\tau^{1 / 2}\left|q_{r}\right| \lesssim(r(s)+r)^{-1} \tau^{\frac{1}{2}} q
$$

Thus it remains to show that

$$
(r(s)+r)^{-1} \tau^{\frac{1}{2}} q \lesssim \delta q_{\delta} b^{2}\left(1+\tau^{-1} r^{-2} \lambda^{2}\right)^{\frac{1}{2}}
$$

Optimizing with respect to $\lambda$ it suffices to consider the cases $\lambda=0$ respectively $\lambda=r \tau^{\frac{1}{2}}$, where the above inequality becomes

$$
(r(s)+r)^{-1} \tau^{\frac{1}{2}}\left(b+b_{\perp}\right) \lesssim \delta\left(b_{\delta}+b\right) b^{2}
$$

or equivalently

$$
\left(b+b_{\perp}\right) b_{1}^{2} \lesssim \delta\left(b_{\delta}+b\right) b^{2}
$$

which is true since by (6.27) and (6.28) we have $b_{\perp} b_{1} \lesssim b^{1}$ while $b_{1} \lesssim \delta b_{\delta}$.

I.(2). Next we consider the $b_{\delta}$ component of $q_{\delta}$, for which it suffices to prove that

$$
\left|b_{\delta r r}\right|+r^{-1}\left|b_{\delta r}\right|+\left|b_{\delta s}\right|+\tau^{1 / 2}\left|b_{\delta r}\right| \lesssim \delta b_{\delta} b^{2}
$$

I.(2).(a). For the $s$ derivative we compute

$$
\frac{b_{\delta s}}{b_{\delta}}=\frac{\left(r_{\delta}^{2}(s)\right)_{s}}{r^{2}+r_{\delta}^{2}(s)}
$$

therefore we want to show that

$$
\left(r_{\delta}^{2}(s)\right)_{s} \lesssim \delta\left(r^{2}+r_{\delta}^{2}(s)\right) b^{2}
$$

We optimize the right hand side with respect to $r$. The minimum is attained when $r^{2}=\min \left\{r_{\delta}^{2}(s), \tau\right\}$. We need to consider two cases: 
I.(2).(a).(i). If $r_{\delta}(s) \lesssim \tau^{\frac{1}{2}}$ then $r_{\delta}^{2}(s) \approx \delta^{-8} r^{2}(s)$ and $\tau>\delta^{-8} r^{2}(s)$. Hence using the estimate from below in (6.27) and (6.28) we obtain $b^{4}\left(r_{\delta}\right) \gtrsim \delta^{-2}$. Then the above bound for $r=r_{\delta}$ follows since $\left|\left(r_{\delta}(s)^{-2}\right)_{s}\right| \lesssim r_{\delta}(s)^{-2}$.

I.(2).(a).(ii). If $r_{\delta}(s) \gtrsim \tau^{\frac{1}{2}}$ then by 6 (6.28) we evaluate $b^{2}\left(\tau^{\frac{1}{2}}\right) \approx \tau^{\frac{1}{4}} r(s)^{-\frac{1}{2}}$. Then the above bound becomes

$$
\delta^{8}\left(r(s)^{-2}\right)_{s} \lesssim \delta r_{\delta}^{-2} \tau^{\frac{1}{4}} r(s)^{-\frac{1}{2}}
$$

Since $\left|\left(r(s)^{-2}\right)_{s}\right| \lesssim r(s)^{-2}$ it suffices to show that

$$
\delta^{8} r(s)^{-2} \lesssim \delta r_{\delta}^{-2} \tau^{\frac{1}{4}} r(s)^{-\frac{1}{2}}
$$

The worst case is $r(s)^{2}=\delta^{6} \tau, r_{\delta}(s)=\delta^{-2} \tau$ when it is verified directly.

I.(2).(b). For the $r$ derivatives the last term is the worst. Since

$$
\frac{b_{\delta r}}{b_{\delta}}=\frac{2 r}{r^{2}+r_{\delta}^{2}(s)}
$$

we want to show that

$$
\tau^{\frac{1}{2}} r \lesssim \delta\left(r^{2}+r_{\delta}^{2}(s)\right) b^{2}
$$

Optimizing with respect to $r$ the worst case is when $r^{2}=\min \left\{r_{\delta}^{2}(s), \tau\right\}$.

I.(2).(b).(i). If $r_{\delta}^{2}(s) \lesssim \tau$ then $r_{\delta}(s) \approx \delta^{-4} r(s)$ therefore for $r=r_{\delta}(s)$ the above relation becomes

$$
\tau^{\frac{1}{2}} \lesssim \delta^{-3} r(s) b^{2}\left(r_{\delta}(s)\right)
$$

which follows from the bound from below in (6.27) and (6.28).

I.(2).(b).(ii). If $r_{\delta}^{2}(s) \gtrsim \tau$ then as before we evaluate $b^{2}\left(\tau^{\frac{1}{2}}\right) \approx \tau^{\frac{1}{4}} r(s)^{-\frac{1}{2}}$ and rewrite the above bound as

$$
\tau \lesssim \delta r_{\delta}^{2}(s) \tau^{\frac{1}{4}} r(s)^{-\frac{1}{2}}
$$

The right hand side is smallest either when $r_{\delta}(s)=\tau^{\frac{1}{2}}$ and $r(s)=\delta^{4} \tau^{\frac{1}{2}}$ or when $r_{\delta}(s)=\delta^{-1} \tau^{\frac{1}{2}}$ and $r(s)=\tau^{\frac{1}{2}}$. In both cases the inequality is easily verified.

II. Now we deal with the general case, which we treat as a perturbation. Since we do not care about the dependence of the constants on $\delta$ to keep the notations simple we include $b_{\delta}$ in $b$ and work with $Q$ instead of $Q_{\delta}$. Thus in the computations below we allow the implicit constants to depend on $\delta$.

Suppose that $A$ is a pseudodifferential operator of order 1 and let $\eta$ be any Lipschitz function. Then

$$
\|[A, \eta] f\|_{L^{2}} \lesssim\|f\|_{L^{2}}
$$

We write

$$
q(\lambda)=b+\left(b^{4}+r^{-2} b_{\perp}^{4} \lambda^{2} / \tau\right)^{\frac{1}{4}}-b=: b+q_{1}(\lambda) .
$$

Even though $q_{1}$ has order $\frac{1}{2}$, we treat it as an operator of order 1 and estimate

$$
\sup _{\lambda}\langle\lambda\rangle^{k-1}\left|q_{1}{ }^{(k)}(\lambda)\right| \lesssim \frac{b_{\perp}^{2}}{r b \tau^{\frac{1}{2}}}
$$

\footnotetext{
${ }^{6}$ the equality holds on the right when $r^{2}=\tau$
} 
Hence, for each $r$ we obtain the bound on the sphere $S^{n-1}$

$$
\|[Q, \eta] f\|_{L^{2}} \lesssim \frac{b_{\perp}^{2}}{r b \tau^{\frac{1}{2}}}\|\eta\|_{L i p\left(S^{n-1}\right)}\|f\|_{L^{2}} .
$$

As a consequence, it also follows that

$$
\left\|\left[Q, \eta \nabla_{\theta}^{j}\right] f\right\|_{L^{2}} \lesssim \frac{b_{\perp}^{2}}{r b \tau^{\frac{1}{2}}}\|\eta\|_{L i p\left(S^{n-1}\right)}\left\|\nabla_{\theta}^{j} f\right\|_{L^{2}}
$$

where $\nabla_{\theta}$ stands for the vector fields $x_{i} \partial_{j}-x_{j} \partial_{i}$ generating the tangent space of $S^{n-1}$.

To use these bounds we write the difference $P_{\psi}-P_{\psi}^{0}$ in polar coordinates,

$$
P_{\psi}-P_{\psi}^{0}=P_{\theta}^{0} \partial_{r}^{2}+P_{\theta}^{1} \partial_{r}+P_{\theta}^{2}
$$

where $P_{\theta}^{j}$ are spherical differential operators of order $j$. Modulo zero homogeneous coefficients which are polynomials in $x r^{-1}$ we can write

$$
\begin{gathered}
P_{\theta}^{0}=d, \quad P_{\theta}^{1}=d r^{-1} \nabla_{\theta}+\tau^{\frac{1}{2}} d+d_{y} \\
P_{\theta}^{2}=d r^{-2} \nabla_{\theta}^{2}+\left(\tau^{\frac{1}{2}} d+d_{y}\right) r^{-1} \nabla_{\theta}+\tau d+\tau^{\frac{1}{2}} d_{y}
\end{gathered}
$$

where $d$ stands for coefficients satisfying (6.22). In the support of $b_{\perp}$ we have $a \approx b$ therefore our regularity assumptions on $d$ show that for fixed $r$ we have

$$
\|d\|_{L^{\infty}}+\|d\|_{L i p\left(S^{n-1}\right)} \lesssim \delta b^{4} \tau^{-1}
$$

The coefficients involving $d_{y}$ satisfy better Lipschitz bounds and are neglected in the sequel.

We expand the commutator

$$
\left[Q, P_{\psi}\right]=\sum_{j=0,1,2}\left[Q, P_{\theta}^{j}\right] \partial_{r}^{2-j}+P_{\theta}^{0}\left(Q_{r r}+2 Q_{r} \partial_{r}\right)+P_{\theta}^{1} Q_{r}
$$

Using the trivial $b^{-1}$ bound for $Q^{-1}$ and (6.17), (6.40) we estimate the first term,

$$
\sum_{j=0,1,2}\left\|Q^{-1}\left[Q, P_{\theta}^{j}\right] \partial_{r}^{2-j} w\right\|_{L^{2}} \lesssim b^{-1} \delta b^{4} \tau^{-1} \frac{b_{\perp}^{2}}{r b \tau^{\frac{1}{2}}} \sum_{j=0,1,2} \tau^{1-\frac{j}{2}}\left\|D^{j} w\right\|_{L^{2}}
$$

This is bounded by the right hand side in (6.35) since

$$
b_{\perp}^{2} \lesssim r \tau^{\frac{1}{2}}
$$

The second term in the commutator is estimated by

$$
\left\|Q^{-1} P_{\theta}^{0}\left(Q_{r r}+2 Q_{r} \partial_{r}\right) w\right\|_{L^{2}} \lesssim \delta\left(\left\|Q_{r r} w\right\|_{L^{2}}+\left\|Q_{r} \partial_{r} w\right\|_{L^{2}}\right)
$$

This is bounded by the right hand side in (6.35) provided that

$$
\left|q_{r r}\right|+\tau^{-\frac{1}{2}}\left|q_{r}\right| \lesssim b^{2}\left(1+\tau^{-\frac{1}{2}} r^{-1} \lambda\right)
$$

which follows from (6.36). The third term in the commutator is treated similarly. This concludes the proof of the proposition. 
To conclude our study of the $L^{2}$ Carleman estimates we need to also pay some attention to elliptic estimates. The conjugated operator $P_{\psi}$ is elliptic in the region $\left\{y^{2}+\xi^{2} \geq 4 \tau\right\}$. Precisely, in this region we have the symbol bound

$$
\left|L_{\psi}^{r}(s, y, \xi)\right| \gtrsim y^{2}+\xi^{2}
$$

Consequently, we can improve our estimates in this region. We consider a smooth symbol $a_{e}(y, \xi)$ with the following properties

$$
\begin{aligned}
& \operatorname{supp} a_{e} \subset\left\{y^{2}+\xi^{2} \geq 8 \tau\right\} \\
& a_{e}(y, \xi)=\left(y^{2}+\xi^{2}\right)^{\frac{1}{2}} \quad \text { in } \quad\left\{y^{2}+\xi^{2} \geq 9 \tau\right\}
\end{aligned}
$$

We define the space $X_{2}$ with norm

$$
\|v\|_{X_{2}}^{2}=\|v\|_{X_{2}^{0}}^{2}+\left\|a_{e}^{w}(y, D) v\right\|^{2}
$$

The dual space $X_{2}^{*}$ has norm

$$
\|f\|_{X_{2}^{*}}^{2}=\inf \left\{\left\|f_{1}\right\|_{\left(X_{2}^{0}\right)^{*}}^{2}+\left\|f_{2}\right\|^{2} ; f=f_{1}+a_{e}^{w}(y, D) f_{2}\right\}
$$

We note that due to the elliptic bound for high frequencies, we also have the dual bounds

$$
\tau^{-\frac{1}{2}}\|b D v\| \lesssim\|v\|_{X^{2}}, \quad\|\nabla f\|_{X_{2}^{*}} \lesssim\left\|b^{-1} f\right\|
$$

Then our final $L^{2}$ Carleman estimate is

Theorem 5. Assume that the coefficients of P satisfy (6.22). Let $\psi$ be as in (6.9) with $h, \phi$ as in Lemmas 6.2,6.3. Then the following $L^{2}$ Carleman estimate holds for all functions $u$ for which the right hand side is finite:

$$
\left\|e^{\psi(s, y)} u\right\|_{X_{2}} \lesssim\left\|e^{\psi(s, y)} P u\right\|_{X_{2}^{*}}
$$

Proof. We first prove the result using the stronger assumption (6.22) on the coefficients. After conjugation we have to show that

$$
\|v\|_{X_{2}^{0}} \lesssim\left\|P_{\psi} v\right\|_{X_{2}^{*}}
$$

We consider two overlapping smooth cutoff symbols $\chi_{i}=\chi_{i}\left(y^{2}+\xi^{2}\right)$ and $\chi_{e}=\chi_{\varepsilon}\left(y^{2}+\xi^{2}\right)$. The interior one $\chi_{i}$ is supported in $\left\{y^{2}+\xi^{2} \leq 7 \tau\right\}$ and equals 1 in $\left\{y^{2}+\xi^{2} \geq 6 \tau\right\}$. The exterior one $\chi_{e}$ is supported in $\left\{y^{2}+\xi^{2} \geq 4 \tau\right\}$ and equals 1 in $\left\{y^{2}+\xi^{2} \leq 5 \tau\right\}$. We need the following bounds for $\chi_{i}$ and $\chi_{e}$ :

Lemma 6.8. a) The operator $\chi_{i}(x, D)$ satisfies the bound

$$
\left\|\chi_{i}(x, D) f\right\|_{\left(X_{2}^{0}\right)^{*}} \lesssim\|f\|_{X_{2}^{*}}
$$

b) The operators $\chi_{i}(x, D)$ and $\chi_{e}^{w}(x, D)$ satisfy the following commutator estimates:

$$
\begin{gathered}
\left\|b^{-1}\left[\chi_{i}(x, D), P_{\psi}\right] v\right\| \lesssim \tau^{-\frac{1}{4}}\|b v\|+\left\|\chi_{e} v\right\| \\
\left\|\left[\chi_{e}^{w}, P_{\psi}\right] v\right\| \lesssim \tau^{\frac{1}{8}}\|b v\|
\end{gathered}
$$


Proof. a) By duality the bound (6.46) is equivalent to

$$
\left\|\chi_{i}(x, D) v\right\|_{X_{2}} \lesssim\|v\|_{X_{2}^{0}}
$$

We have

$$
\left\|a_{e}(x, D) \chi_{i}(x, D) v\right\| \lesssim \tau^{-N}\|f\|
$$

since the supports of the symbols $\left(1-\chi_{e}(x, \xi)\right)$ and $a_{e}(x, \xi)$ are $O\left(\tau^{\frac{1}{2}}\right)$ separated. Then it remains to show that

$$
\left\|\chi_{i}(x, D) v\right\|_{X_{2}^{0}} \lesssim\|v\|_{X_{2}^{0}}
$$

which is fairly straightforward and is left for the reader.

b) We now consider the bound (6.47). Commute first $\chi_{i}$ with $\partial_{s}+H-h^{\prime}(s)$. We have

$$
\left[\chi_{i}(x, D), \partial_{s}+H-h^{\prime}(s)\right]=\left[\chi_{i}(x, D), H\right]
$$

Since $\chi_{e}=1$ in the support of $\nabla_{x, \xi} \chi_{i}$ and the Poisson bracket of $\chi_{i}$ and $x^{2}+\xi^{2}$ vanishes, by standard pdo calculus we obtain

$$
\left\|\left[\chi_{i}(x, D), \partial_{s}+H-h^{\prime}(s)\right] v\right\| \lesssim\left\|\chi_{e} v\right\|+\tau^{-N}\|v\|
$$

The difference $P_{\psi}-\left(\partial_{s}+H-h^{\prime}(s)\right)$ can be expressed in the form

$$
P_{\psi}-\left(\partial_{s}+H-h^{\prime}(s)\right)=\partial g \partial+\tau^{\frac{1}{2}}(g \partial+\partial g)+\tau g
$$

where the function $g$ satisfies the bounds

$$
|g|+\langle y\rangle\left|g_{y}\right|+\langle y\rangle\left|g_{y y}\right| \lesssim \varepsilon_{i}
$$

These lead to an estimate for fixed $s \in[i, i+1]$,

$$
\left\|b^{-1}\left[\chi_{i}(x, D), P_{\psi}-\left(\partial_{s}+H-h^{\prime}(s)\right)\right] v\right\| \lesssim \varepsilon_{i} \tau^{\frac{1}{2}}\left\|\langle y\rangle^{-1} b^{-1} v\right\|
$$

Then (6.47) follows since

$$
\varepsilon_{i} \tau^{\frac{1}{2}}\langle y\rangle^{-1} \lesssim \tau^{-\frac{1}{4}} b^{2}
$$

Finally, the proof of the estimate (6.48) is similar but simpler.

We continue with the proof of the proposition. For the nonelliptic part we apply (6.32) to the function $\chi_{i}(x, D) v$ which is supported in $\left\{y^{2}<9 \tau\right\}$. This gives

$$
\left\|\chi_{i}(x, D) v\right\|_{X_{2}^{0}} \lesssim\left\|\chi_{i}(x, D) P_{\psi} v\right\|_{\left(X_{2}^{0}\right)^{*}}+\left\|\left[\chi_{i}(x, D), P_{\psi}\right] v\right\|_{L^{2}}
$$

For the first term on the right we use the bound (6.46) while for the second we use (6.47). This yields

$$
\left.\| \chi_{i}(x, D)\right) v\left\|_{X_{2}^{0}} \lesssim\right\| P_{\psi} v\left\|_{X_{2}^{*}}+\tau^{-\frac{1}{4}}\right\| b v\|+\| \chi_{e} v \|
$$

On the other hand for the estimate in the elliptic region we compute

$$
\left\langle\left(\chi_{e}^{w}\right)^{2} v, P_{\psi} v\right\rangle=\underset{40}{\left\langle\chi_{e}^{w} v, L_{\psi}^{r} \chi_{e}^{w} v\right\rangle+\left\langle\chi_{e}^{w} v,\left[\chi_{e}^{w}, P_{\psi}^{r}\right] v\right\rangle}
$$


For the first term we split $L_{\psi}^{r}$ into $H-h^{\prime}$ plus a perturbation. Using pointwise bounds for the coefficients of $P_{\phi}$ we obtain

$$
\left|L_{\psi}^{r} v-\left(H-h^{\prime}\right) v\right| \lesssim \delta_{1}\left(\left(\tau+y^{2}\right)|v|+\tau^{\frac{1}{2}}|D v|+\left|D^{2} v\right|\right)
$$

which shows that

$$
\left\langle\chi_{e}^{w} v, L_{\psi}^{r} \chi_{e}^{w} v\right\rangle=\left\langle\chi_{e}^{w} v,\left(H-h^{\prime}\right) \chi_{e}^{w} v\right\rangle+O\left(\delta_{1}\left\langle\chi_{e}^{w} v,(H+\tau) \chi_{e}^{w} v\right\rangle\right)
$$

The symbol of $H-h^{\prime}$ is elliptic in the support of $\chi_{e}$, therefore a standard elliptic argument yields

$$
\left\langle\chi_{e}^{w} v,(H+\tau) \chi_{e}^{w} v\right\rangle \lesssim\left\langle\chi_{e}^{w} v,\left(H-h^{\prime}\right) \chi_{e}^{w} v\right\rangle+C \tau^{-N}\|v\|^{2}
$$

for a large constant $C$. This further gives

$$
\left\langle\chi_{e}^{w} v,(H+\tau) \chi_{e}^{w} v\right\rangle \lesssim\left\langle\chi_{e}^{w} v, L_{\psi}^{r} \chi_{e}^{w} v\right\rangle+C \tau^{-N}\|v\|^{2}
$$

Returning to (6.50), we obtain

$$
c\left\langle\chi_{e}^{w} v,(H+\tau) \chi_{e}^{w} v\right\rangle \leq-\left\langle\left(\chi_{e}^{w}\right)^{2} v, P_{\psi} v\right\rangle+\left\langle\chi_{e}^{w} v,\left[\chi_{e}^{w}, P_{\psi}^{r}\right] v\right\rangle+C \tau^{-N}\|v\|^{2}
$$

We use (6.48) and then the Cauchy-Schwartz inequality to obtain

$$
\left\langle\chi_{e}^{w} v,(H+\tau) \chi_{e}^{w} v\right\rangle \lesssim\left\|(H+\tau)^{-\frac{1}{2}} P_{\psi} v\right\|^{2}+\tau^{-\frac{3}{4}}\|b v\|^{2}
$$

The first term on the right is properly controlled due to the straightforward estimate

$$
\left\|(H+\tau)^{-\frac{1}{2}} f\right\| \lesssim\|f\|_{X_{2}^{*}}
$$

Hence combining the above inequality with (6.49) we obtain

$$
\left.\left.\| \chi_{i}^{(} x, D\right)\right) v\left\|_{X_{2}^{0}}+\right\|(H+\tau)^{\frac{1}{2}} \chi_{e}^{w}(x, D) v\|\lesssim\| P_{\psi} v\left\|_{X_{2}^{*}}+\tau^{-\frac{3}{4}}\right\| b v\|+\| \chi_{e}^{w}(x, D) v \|
$$

The last two terms on the right are negligible compared to the left hand side, therefore we obtain

$$
\|v\|_{X_{2}^{0}}+\left\|(H+\tau)^{\frac{1}{2}} \chi_{e}^{w}(x, D) v\right\| \lesssim\left\|P_{\psi} v\right\|_{X_{2}^{*}}
$$

Then (6.45) follows since $\chi_{e}=1$ in the support of $a_{e}$.

It remains to show that the assumption (6.22) on the coefficients for (6.7) can be replaced by the weaker condition (1.15). This is a direct consequence of (6.43) combined with the following regularization result:

Lemma 6.9. Let $d$ be a function which satisfies (1.15). Then there is an approximation $g_{1}$ of it satisfying (6.22) so that

$$
\left|g-g_{1}\right| \lesssim b^{2} \tau^{-1}
$$

Proof. First we transfer (1.15) to the $(s, y)$ coordinates. A short computation yields the equivalent form

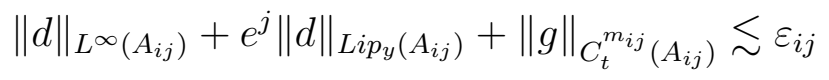

where the new continuity modulus $\tilde{m}_{i j}$ is given by

$$
\tilde{m}_{i j}(\rho)=\underset{41}{\rho}+e^{-\frac{2 j}{3}} \rho^{\frac{1}{3}}
$$


Within $A_{i j}$ we regularize $d$ in $y$ on the $\delta y=\tau^{-\frac{1}{2}}$ scale and in $s$ on the $\delta s=e^{\frac{j}{2}} \tau^{-\frac{3}{4}}$ scale,

$$
d_{1}=S_{<\tau^{\frac{1}{2}}}\left(D_{y}\right) S_{<e^{\frac{j}{2}} \tau^{-\frac{3}{4}}}\left(D_{s}\right) d
$$

These localized regularizations are assembled together using a partition of unit corresponding to $A^{i j}$. In $A_{i j}$ we compute

$$
\left|d-d_{1}\right| \lesssim \varepsilon_{i j}\left(e^{-j} \delta y+m_{i j}(\delta s)\right) \approx \varepsilon_{i j}\left(e^{-j} \tau^{-\frac{1}{2}}+e^{-\frac{j}{2}} \tau^{-\frac{1}{4}}\right) \lesssim \varepsilon_{i j}^{\frac{1}{2}} e^{-\frac{j}{2}} \tau^{-\frac{1}{4}} \lesssim b^{2} \tau^{-1}
$$

while

$$
\left|\partial_{s} g_{1}\right| \lesssim \varepsilon_{i j} \frac{m_{i j}(\delta s)}{\delta s} \approx \varepsilon_{i j} e^{-j} \tau^{\frac{1}{2}}
$$

The bounds for higher order derivatives of $g_{1}$ follow trivially due to the frequency localization.

\section{7. $L^{p}$ Carleman estimates for variable Coefficient operators}

The variable coefficient counterpart of Proposition 4 uses the more convex weights constructed in Section 6. For convenience we write it in the $(s, y)$ coordinates. Let $\tau>>1$ and $\mathcal{B}(\tau)$ be as in (1.25), (1.26) and (1.27).

We define the function space $X$ through its norm

$$
\|v\|_{X}:=\|v\|_{X_{2}}+\|v\|_{l^{2}\left(\mathcal{B}(\tau) ; L_{t}^{\infty} L_{x}^{2}\right)}+\|v\|_{l^{2}\left(\mathcal{B}(\tau) ; L_{t}^{p} L_{x}^{q}\right)}
$$

where $(p, q)$ is an arbitrary Strichartz pair, with $X_{2}$ as defined in (6.41).

Its (pre)dual space has the norm

$$
\|f\|_{X^{*}}=\inf _{f=f_{1}+f_{2}+f_{3}}\left\|f_{1}\right\|_{X_{2}^{*}}+\left\|f_{2}\right\|_{L^{1} L^{2}}+\left\|f_{3}\right\|_{L^{p^{\prime}} L^{q^{\prime}}}
$$

Then we have the following improvement of Theorem 5 .

Theorem 6. There exists $\psi$ as in (6.9) with $h$ and $\phi$ as in Lemma 6.2 and 6.3. Then the following estimate holds for all compactly supported sufficiently regular functions $u$.

$$
\left\|e^{\psi} u\right\|_{X} \lesssim\left\|e^{\psi} \tilde{P} u\right\|_{X^{*}}
$$

The relation between $\psi$ and the partition $\mathcal{B}(\tau)$ remains a bit mysterious at this level. If we replace it by the empty partition then the statement remains true for all $\psi$ with $h$ and $\phi$ as in Lemma 6.2. The same is true for a partition into time slices of size 1 . The convexity properties of $\phi$ allow a localization to the finer partition $B_{i j}$ as in 6.6 (and, as we shall soon see, to an even finer partition). q It is possible to choose $\phi$ and $h$ so that the partition $\left(B_{i j}\right)$ is finer than the one defined by $\mathcal{B}(\tau)$. We assume in the sequel that $\psi$ has been chosen with these properties. 
Proof. As usual this is equivalent to proving a bound from below for the conjugated operator,

$$
\|v\|_{X} \lesssim\left\|P_{\psi} v\right\|_{X^{*}}
$$

The main step in the proof is to produce a parametrix for $P_{\psi}$. The key point is that the parametrix is allowed to have a fairly large $L^{2}$ error. This is because $L^{2}$ errors can be handled by Theorem 5. The advantage in having a large $L^{2}$ error is that it permits to localize the parametrix construction to relatively small sets, on which we can freeze the coefficients and eventually reduce the problem to the case of the Hermite operator. The properties of the parametrix are summarized in the following

Proposition 7.1. a) Under the assumptions of the theorem there exists a parametrix $T$ for $P_{\psi}$ with the following properties:

$$
\|T f\|_{X} \lesssim\|f\|_{X^{*}}
$$

and

$$
\left\|P_{\psi} T f-f\right\|_{X_{2}^{*}} \lesssim\|f\|_{X^{*}}
$$

b) The same result holds with $P_{\psi}$ replaced by $P_{\psi}^{*}$.

We first use the proposition to conclude the proof of the Theorem. Let

$$
P_{\psi} v=f+g, \quad\|f\|_{X_{2}^{*}}+\|g\|_{L^{1} L^{2}+L^{p^{\prime} L^{q^{\prime}}}} \approx\left\|P_{\psi} v\right\|_{X^{*}}
$$

With $T$ as in part (a) of the proposition we set

$$
w=v-T g, \quad P_{\psi} w=f+g-P_{\psi} T g
$$

By (7.5) we can bound $T g$ in $X$, therefore it suffices to bound $w$ in $X$. On the other hand by (7.6) we obtain

$$
\left\|P_{\psi} w\right\|_{X_{2}^{*}} \lesssim\|f\|_{X_{2}^{*}}+\left\|g-P_{\psi} T g\right\|_{X_{2}^{*}} \lesssim\left\|P_{\psi} v\right\|_{X^{*}}
$$

It remains to show that

$$
\|w\|_{X} \lesssim\left\|P_{\psi} w\right\|_{X_{2}^{*}}
$$

By Theorem 5 we can estimate the $X_{2}$ norm of $w$ and replace this with the weaker bound

$$
\|w\|_{L^{\infty} L^{2} \cap L^{p} L^{q}} \lesssim\|w\|_{X_{2}}+\left\|P_{\psi} w\right\|_{X_{2}^{*}}
$$

This is proved using a duality argument and the parametrix $T$ for $P_{\psi}^{*}$ given by part (b) of the proposition. For $f \in X^{*}$ we write

$$
\begin{aligned}
\langle w, f\rangle & =\left\langle w, P_{\psi}^{*} T f\right\rangle+\left\langle w, f-P_{\psi}^{*} T f\right\rangle \\
& =\left\langle P_{\psi} w, T f\right\rangle+\left\langle w, f-P_{\psi}^{*} T f\right\rangle
\end{aligned}
$$

Using both (17.5) and (7.6) with $P_{\psi}$ replaced by $P_{\psi}^{*}$ we obtain

$$
|\langle w, f\rangle| \lesssim\left(\left\|P_{\psi} w\right\|_{X_{2}^{*}}+\|w\|_{X^{2}}\right)\|f\|_{X^{*}}
$$

and (7.7) follows. This concludes the proof of Theorem 6 . 
It remains to prove the Proposition 7.1,

Proof of Proposition 7.1. The strategy for the proof is simple: On sufficiently small sets we can approximate the problem by one with constant coefficients and the properties of the parametrix follow from Section 4. We use a partition of unity to construct a global parametrix from local ones. We obtain $L^{2}$ errors from

(1) Commuting cutoff functions with the operator. Hence the partition has to be sufficiently coarse.

(2) Approximating the variable coefficient operator by constant coefficient operators. Hence the partition has to be sufficiently fine.

To elaborate on this we define the notion of a local parametrix:

Definition 7.2 (Local parametrix). Given a convex set $B$ we call $T$ a (B-) local parametrix for $P_{\psi}$ if for all $f$ supported in $B$

$$
\begin{gathered}
\|T f\|_{X} \lesssim\|f\|_{X^{*}}, \\
\left\|P_{\psi} T f-f\right\|_{X_{2}^{*}} \lesssim\|f\|_{X^{*}}
\end{gathered}
$$

and $T f$ is supported in $2 B$.

If $T$ is a parametrix and $\eta$ is supported on $2 B, \eta=1$ on $B$ then $\psi T$ is a local parametrix, but with constants depending on the commutator of $P_{\psi}$ and $\eta$. Vice verse, if $\left(B_{j}\right)$ is a covering, $\left(\eta_{j}\right)$ a subordinate partition of 1 and $T_{j}$ are local parametrices then

$$
T=\sum_{j} T_{j} \eta_{j}
$$

is a global parametrix, because (7.8) is obtained by summation, and

$$
P_{\psi} \sum_{j} T_{j}\left(\eta_{j} f\right)-f=\sum_{j}\left(P_{\psi} T_{j} \eta_{j} f-\eta_{j} f\right.
$$

provided

$$
\sum_{j}\left\|\eta_{j} f\right\|_{X^{*}}^{2} \lesssim\|f\|_{X^{*}}^{2}
$$

and its adjoint

$$
\|u\|_{X}^{2} \lesssim \sum_{j}\left\|\eta_{j} u\right\|_{X}^{2}
$$

This is obvious for the $L^{2}$ part and has to be checked for the other part. This strategy of constructing local parametrices leads, if it is possible, to estimates which are stronger than in Proposition 7.1 and Theorem 6 , because we may replace the function space $X$ by $l^{2} X\left(B_{j}\right)$ respectively. $l^{2} X^{*}\left(B_{j}\right)$.

In the first part of the proof we study the localization, and in the second part we provide the local parametrices. 
7.1. Localization scales. Here we introduce a localization scale which is finer than the $B_{i j}$ partition of the space, and show that it suffices to construct the parametrix in each of these smaller sets. Precisely, the sets $B_{i j}^{k}$ introduced below are the smallest sets to which one can localize the $L^{2}$ estimates for the operator $P_{\psi}$. The choice of their size is not yet apparent at this point, but will become clear in the very last step of the proof, where we estimate the commutator of $P_{\psi}$ with cutoff functions on such sets. We consider three cases depending on the size of $\varepsilon_{i}$.

(1) If $\varepsilon_{i} \leq \tau^{-1}$ then we use $B_{i 0}$ as it is.

(2) If $\tau^{-1} \leq \varepsilon_{i} \leq \tau^{-\frac{1}{2}}$ then we partition the set $B_{i 0}$ into time slices $B_{i 0}^{k}$ of thickness

$$
\delta s=b_{i 0}^{-2} .
$$

(3) If $\tau^{-1} \leq \varepsilon_{i}$ and $j \neq 0$ then we partition $B_{i j}$ into subsets $B_{i j}^{k}$ which have the time scale, radial scale and angular scale given by

$$
\delta s=b_{i j}^{-2}, \quad \delta y=\tau^{\frac{1}{2}} b_{i j}^{-2}, \quad \delta y^{\perp}=\tau^{\frac{1}{2}} b_{i j, \perp}^{-2}
$$

This gives a decomposition of the space

$$
\mathbb{R} \times \mathbb{R}^{n}=\bigcup B_{i j}^{k}
$$

We also consider a subordinated partition of unity

$$
1=\sum \chi_{i j}^{k}
$$

Suppose that in each set $B_{i j}^{k}$ we have a parametrix $T_{i j}^{k}$ satisfying (7.5) and (7.6). Then we define the global parametrix $T$ by

$$
T=\sum T_{i j}^{k} \chi_{i j}^{k}
$$

We have by an iterated application of Minkowski's inequality

$$
\left\|\chi_{i j}^{k} f\right\|_{l^{2}\left(L^{1} L^{2}+L^{p^{\prime}} L^{q^{\prime}}\right)} \lesssim\|f\|_{L^{1} L^{2}+L^{p^{\prime}} L^{q^{\prime}}}
$$

and the dual bound

$$
\left\|\sum T_{i j}^{k} \chi_{i j}^{k} f\right\|_{L^{\infty} L^{2} \cap L^{p} L^{q}} \lesssim\left\|T_{i j}^{k} \chi_{i j}^{k} f\right\|_{l^{2}\left(L^{\infty} L^{2} \cap L^{p} L^{q}\right)}
$$

Hence (7.5) for $T$ would follow if we proved that

$$
\left\|\sum v_{i j}^{k}\right\|_{X_{2}} \lesssim\left\|v_{i j}^{k}\right\|_{l^{2} X_{2}}
$$

where $v_{i j}^{k}=T_{i j}^{k} \chi_{i j}^{k} f$ are supported in $B_{i j}^{k}$. This is trivial by orthogonality for the $L^{2}$ component of the $X_{2}$ norm. It is also straightforward for the elliptic part since the kernel of the operator in the following sense: Let $\chi_{0} \in C^{\infty}(\mathbb{R})$ be supported in $[-3 / 2,3 / 2]$, identically 1 in $[-1,1]$. We define $\chi_{e}(x, \xi)=$ $\chi_{0}\left(5-\left(x^{2}+\xi^{2}\right) / \tau^{2}\right)$. Then

$$
\left\|\sum \chi_{e}^{w} v_{i j}^{k}\right\|_{X_{2}} \lesssim\left\|\chi_{e}^{w} v_{i j}^{k}\right\|_{l^{2} X_{2}}
$$


and the adjoint estimate holds since the kernel of $\chi_{e}^{w}$ is rapidly decreasing beyond the $\tau^{-\frac{1}{2}}$ scale, which is much smaller than the smallest possible spatial size for $B_{i j}^{k}$, namely $\delta y \gtrsim \tau^{-\frac{1}{4}}$.

It remains to consider the angular part of the $X_{2}$ norm, which is best described using the spherical multiplier $Q$ appearing in Proposition 6.7. The symbol of $Q$ is smooth with respect to $\lambda$ on the $\tau^{\frac{1}{2}} r b^{2} b_{\perp}^{-2}$ scale therefore its kernel is rapidly decaying on the angular scale $\delta \theta=\tau^{-\frac{1}{2}} r^{-1} b^{-2} b_{\perp}^{2}$ which corresponds to $\delta y_{\perp}=\tau^{-\frac{1}{2}} b_{\perp}^{2} b^{-2}$. But by (3) and (6.5) this can be no larger than $\tau^{-\frac{3}{8}}$ which is again much smaller than the smallest possible spatial size for $B_{i j}^{k}$. Thus orthogonality arguments still apply.

Then we have

$$
\left[P_{0}, \chi^{i j}\right] u=2 \chi_{y}^{i j} u_{y}+\chi_{y y}^{i j} u+\chi_{s}^{i j} u
$$

We claim that the right hand side is negligible in the estimate. For this it suffices to verify that

$$
\left|\chi_{y}^{i j}\right| \ll \tau^{-\frac{1}{2}} b_{i j}^{2}, \quad\left|\chi_{y y}^{i j}\right| \ll b_{i j}^{2}, \quad\left|\chi_{s}^{i j}\right| \ll b_{i j}^{2}
$$

The last relation is trivial. For the first two we consider three cases.

(1) If $j=0$ and $\varepsilon_{j}<C \tau^{-1}$ then we need no spatial truncation. We are allowed to truncate at $|y|>C \tau^{\frac{1}{2}}$ to separate the elliptic region, though.

(2) If $j=0$ and $\varepsilon_{j}>C \tau^{-1}$ then

$$
\left|\chi_{y}^{i j}\right| \lesssim e^{-j(i)}, \quad\left|\chi_{y y}^{i j}\right| \lesssim e^{-2 j(i)}
$$

while

$$
b_{i 0}^{4}=a_{i j(i)}^{4}=\varepsilon_{i j(i)} \tau^{\frac{3}{2}} e^{-j(i)} \approx C e^{-2 j(i)} \tau
$$

(3) Otherwise,

$$
\left|\chi_{y}^{i j}\right| \lesssim e^{-j}, \quad\left|\chi_{y y}^{i j}\right| \lesssim e^{-2 j}
$$

while

$$
b_{i j}^{4}=a_{i j}^{4}=\varepsilon_{i j} \tau^{\frac{3}{2}} e^{-j} \gtrsim C e^{-2 j(i)} \tau
$$

The results can be summarized by saying that it suffices to construct local parametrices in the sets $B_{i j}^{k}$.

7.2. Freezing coefficients. Our first observation is that restricting the result in the proposition to a single region $B_{i j}^{k}$ allows us to freeze the weights $b, b_{\perp}$ in the $X_{2}$ norms.

Next we are interested in freezing the coefficients of $P$. We consider the same three cases as above:

The case $\varepsilon_{i} \leq \tau^{-1}$. In this case we are localized to

$$
B_{i 0}=[i, i+1] \times \mathbb{R}^{n}
$$

and we have

$$
b_{i 0} \approx 1, \quad{ }_{46} \varepsilon_{i j} \lesssim \tau^{-1}
$$


By (6.2) the second relation leads to

$$
|d| \lesssim \tau^{-1}
$$

Then using also (6.43) we can estimate the terms involving $d$ in the expression (6.22) for $P_{\psi}$,

$$
\|\partial d \partial v\|_{X_{2}^{*}}+\tau\|d v\|_{X_{2}^{*}}+\tau^{\frac{1}{2}}\|(d \partial+\partial d) v\|_{X_{2}^{*}} \lesssim\|v\|_{X_{2}}
$$

Hence without any restriction of generality we can assume that $d=0$ in $P_{\psi}$, which corresponds to taking $g=I_{n}$.

We also observe that in this case we have

$$
|\phi| \lesssim 1, \quad\left|\phi_{y}\right| \lesssim \tau^{-\frac{1}{2}}
$$

Then we can also drop the $\phi$ component of $\psi$. Finally, since

$$
\left|h_{s s}\right| \lesssim 1
$$

we can replace $h$ by its linearization at some point in the corresponding $s$ region.

Conclusion: It suffices to prove the result when $d=0, \psi(y, s)=\tau s$.

We note that the separation of $\tau$ from integers is no longer needed due to the localization to unit $s$ intervals.

The case $\tau^{-1} \leq \varepsilon_{i}$. In this case we are localized to a region of the form

$$
B_{i 0}^{k}=\left[s_{0}, s_{0}+e^{j(i)} \tau^{-\frac{1}{2}}\right] \times B\left(0, e^{j(i)}\right)
$$

and we have

$$
b_{i 0}^{4} \approx \tau e^{-2 j(i)}, \quad \varepsilon_{i j} \lesssim e^{-j(i)} \tau^{-\frac{1}{2}}
$$

The second relation leads to

$$
|d| \lesssim e^{-j(i)} \tau^{-\frac{1}{2}}
$$

Then (7.10) is still valid, so we can assume again that $d=0$ in $P_{\psi}$.

We also observe that in this case we have

$$
|\phi| \lesssim 1, \quad\left|\phi_{y}\right| \lesssim e^{-j(i)}
$$

Then we can also drop the $\phi$ component of $\psi$. Finally, since

$$
\left|h_{s s}\right| \lesssim e^{-j(i)} \tau^{-\frac{1}{2}}
$$

we can replace $h$ by its linearization at some point in the corresponding $s$ region.

Conclusion: It suffices to prove the result when $d=0, \psi(y, s)=\tau s$. 
The case $\tau^{-1} \leq \varepsilon_{i}$. In this case we are localized to a region of the form

$$
B_{i j}^{k}=\left[s_{0}, s_{0}+\tau^{-\frac{3}{4}} e^{\frac{j}{2}} \varepsilon_{i j}^{-\frac{1}{2}}\right] \times B\left(y_{0}, \tau^{-\frac{1}{4}} e^{\frac{j}{2}} \varepsilon_{i j}^{-\frac{1}{2}}\right), \quad\left|y_{0}\right| \approx e^{j}
$$

and we have

$$
b_{i j}^{2} \approx \varepsilon_{i j}^{\frac{1}{2}} \tau^{\frac{3}{4}} e^{-\frac{j}{2}}
$$

Using (6.2) it follows that

$$
\left|g(s, y)-g\left(s_{0}, y_{0}\right)\right| \lesssim \varepsilon_{i j}^{\frac{1}{2}} \tau^{-\frac{1}{4}} e^{\frac{j}{2}}
$$

Arguing as before, this allows us to freeze $d$ within $B_{i j}^{k}$. However, we note that we are no longer allowed to replace $d$ by 0 .

Next we turn our attention to the weight function $\psi$. First we have

$$
\left|h_{s s}\right| \lesssim \varepsilon_{j} \tau \lesssim \varepsilon_{i j} \tau^{\frac{3}{2}} e^{-j}
$$

which allows us to replace $h$ by its linearization in $s$ at $s_{0}$.

Secondly, we claim that we can replace $\phi$ by its linearization at $y_{0}$. In the radial direction we have weaker localization but a stronger bound

$$
\left|\phi_{r r}\right| \lesssim \varepsilon_{i j} \tau^{\frac{1}{2}} e^{-j}
$$

In the transversal direction we have better localization but a weaker bound,

$$
\left|\phi_{y y}\right| \lesssim \varepsilon_{i} \tau^{\frac{1}{2}} e^{-j}
$$

The first bound allows us to obtain the relation

$$
\left|\phi_{y}^{2}(y, s)-\phi_{y}^{2}\left(y_{0}, s_{0}\right)\right| \lesssim \varepsilon_{i j}^{-\frac{1}{2}} \tau^{\frac{3}{4}} e^{-\frac{j}{2}} \approx b_{i j}^{2}
$$

Using also the second bound we can write

$$
\left(\phi_{y}(y, s)-\phi_{y}\left(y_{0}, s_{0}\right)\right) \partial_{y}=\nu_{r} \partial_{r}+\nu_{\perp} \partial_{\perp}
$$

where the coefficients $\nu_{r}$ and $\nu_{\perp}$ are smooth on the $B_{i j}^{k}$ scale and satisfy the bounds

$$
\left|\nu_{r}\right| \lesssim \tau^{-\frac{1}{2}} b_{i j}^{2}, \quad\left|\nu_{r}\right| \lesssim \tau^{-\frac{1}{2}} b_{i j, \perp}^{2}
$$

Conclusion: It suffices to prove the result when $d=g\left(s_{0}, y_{0}\right), \psi(y, s)=$ $\tau s+c y,|c| \lesssim \varepsilon_{i} \tau^{\frac{1}{2}}$

Additional simplification in the highly localized case. Given the above simplifications we need to work with a constant coefficient operator $P_{\psi}$ which has the form

$$
P_{\psi}=-\partial_{t}+H-\tau+\partial d \partial+c \partial, \quad|d| \lesssim \varepsilon_{i j}, \quad|c| \leq \varepsilon_{i}
$$

We diagonalize the second order part with a linear change of variables to obtain

$$
P_{\psi}=-\partial_{t}+H-\tau+c \partial+O\left(\varepsilon_{i j}\right) y^{2}
$$

We can freeze the last term at $y_{0}$ and add it into $\tau$. To deal with $c$ we make the change of variable

$$
y \rightarrow y-\left(s-s_{0}\right) c
$$


Then our operator becomes

$$
\tilde{P}_{\psi}=-\partial_{t}+\Delta-\left(y-c\left(s-s_{0}\right)\right)^{2}+\tau
$$

and the $s-s_{0}$ terms are negligible due to the $s$ localization.

Conclusion: We can assume without any restriction in generality that $g=I_{n}$ and $\psi=\tau s$.

7.3. The localized parametrix. We begin with the global parametrix $K$ constructed in Section 5. Then we define the parametrix $T_{B}$ in $B$ by

$$
T_{B}=\chi_{2 B} K, \quad B=B_{i j}^{k}
$$

and show that it satisfies (7.8) and (7.9).

The $L^{p}$ part of (7.8) follows directly from (5.4). It remains to prove the $X_{2}$ part,

$$
\left\|T_{B} f\right\|_{X_{2}} \lesssim\|f\|_{L^{1} L^{2}+L^{p^{\prime}} L^{q^{\prime}}}
$$

The elliptic part of the $X_{2}$ bound, namely

$$
\left\|a_{e}^{w}(x, D) \chi_{2 B} K f\right\|_{L^{2}} \lesssim\|f\|_{L^{1} L^{2}+L^{p^{\prime}} L^{q^{\prime}}}
$$

is obtained by an argument which is similar to the one beginning with (6.50).

For the rest we consider two cases.

i) If $j=0$ then $B$ is a ball, and we can use (5.4) directly with $R=d$.

ii) If $j>0$ then $B$ is contained in a sector $B \subset B_{R, d}$ but may be shorter than $R$. This is why we can use (5.4) for the angular part of the $X_{2}$ norm, but not for the $L^{2}$ part. However, the $L^{2}$ part can be always obtained by taking advantage of the time localization,

$$
\left\|b^{i j} T_{B} f\right\|_{L^{2}} \lesssim\left\|T_{B} f\right\|_{L^{\infty} L^{2}} \lesssim\|f\|_{L^{1} L^{2}+L^{p^{\prime}} L^{q^{\prime}}}
$$

It remains to consider the error estimate (7.9). We have

$$
f-\left(\partial_{s}-H+\tau\right) T_{B} f=\left[\chi_{2 B}, \partial_{s}-H+\tau\right] K f=\left[\chi_{2 B}, \partial_{s}-H+\tau\right] \chi_{4 B} K f
$$

But arguing as above $\chi_{4 B} K_{0} f$ satisfies the same $X_{2}$ bound as $\chi_{2 B} K_{0} f$. Hence it suffices to show that

$$
\left[\chi_{2 B}, \partial_{s}-H+\tau\right]: X_{2} \rightarrow X_{2}^{*}
$$

This is where the dimensions of the set $B$ are essential; they are chosen to be minimal so that the above property holds. We have

$$
\begin{aligned}
{\left[\chi_{2 B}, \partial_{s}-H\right.} & +\tau]=-\partial_{s} \chi_{2 B}+\left(\partial_{y} \chi_{2 B}\right) \partial_{y}+\partial_{y}\left(\partial_{y} \chi_{2 B}\right) \\
& =-\partial_{s} \chi_{2 B}+\left(\partial_{r} \chi_{2 B r}\right) \partial_{r}+\partial_{r}\left(\partial_{r} \chi_{2 B r}\right)+\left(\partial_{\perp} \chi_{2 B y}\right) \partial_{\perp}+\partial_{\perp}\left(\partial_{\perp} \chi_{2 B}\right)
\end{aligned}
$$

For the first factor we use the bound

$$
\left|\partial_{s} \chi_{2 B}\right| \lesssim b_{i j}^{2}
$$

For the radial derivatives of $\chi_{2 B}$ we combine (6.43) with

$$
\left|\partial_{r} \chi_{2 B}\right| \lesssim \tau^{-\frac{1}{2}} b_{i j}^{2}
$$


Finally, for the angular derivatives we use the angular $H^{\frac{1}{2}}$ norm in $X_{2}$ and the bound

$$
\left|\partial_{r} \chi_{2 B}\right| \lesssim \tau^{-\frac{1}{2}} b_{i j \perp}^{2}
$$

\section{THE GRADIENT TERM}

In this section we consider the full problem, i.e. involving also the gradient potential $W$. Ideally one might want to have a stronger version of Theorem 6 which includes additional bounds for the gradient, more precisely for

$$
\left\|e^{\psi(s, y)} \nabla u\right\|_{L^{2}}
$$

But such bounds cannot hold, for this would imply that one can improve the $L^{p}$ indices in a restriction type theorem. To overcome this difficulty we proceed as in [17], using Wolff's osculation Lemma. Wolff's idea is that by varying the weight one can ensure concentration in a sufficiently small set, in which the gradient potential term is only as strong as the potential term. Thus we still obtain a one parameter family of Carleman estimates, but with the weight depending not only on the parameter but also on the function we apply the estimate to.

Given a gradient potential $W$ satisfying (1.19), we first readjust the parameters $\varepsilon_{i j}, \varepsilon_{i}$ constructed in Lemma 6.1 in order to insure that we have the additional condition

$$
\|W\|_{L^{n+2}\left(A_{i}^{\tau}\right)} \ll \varepsilon_{i}
$$

Then we begin with the spherically symmetric weights $\psi$ constructed in Section 6 and modify them as follows:

$$
\Psi(s, y)=\psi(s, y)+\delta k(s, y)
$$

where the perturbation $k$ is supported in $\{|y| \leq 9 \tau\}$ and is subject to the following conditions:

$$
\left|\partial_{s}^{\alpha} \partial_{y}^{\beta} \partial_{\perp}^{\beta} k(s, y)\right| \lesssim \varepsilon_{i} \tau^{1-\frac{\alpha}{2}} \quad s \in[i, i+1]
$$

Here $\delta$ is a sufficiently small parameter.

In order to prove the strong unique continuation result in the presence of the gradient potential $W$ we need the following modification of Theorem (6)

Theorem 7. Assume that (1.15) holds. Then for each $\tau>0$ and $W$ subject to

$$
\|W\|_{L^{n+2}\left(A_{i}^{\tau}\right)} \leq \varepsilon_{i}
$$

and each function $u$ vanishing of infinite order at $(0,0)$ and $\infty$ there exists a perturbation $k$ as in (8.2) so that

$$
\left\|e^{\Psi} u\right\|_{X}+\left\|e^{\Psi} W \nabla u\right\|_{X^{*}}+\left\|e^{\Psi} \nabla(W u)\right\|_{X^{*}}+\tau^{\frac{1}{2}}\left\|e^{\Psi} W u\right\|_{X^{*}} \lesssim\left\|e^{\Psi(x)} \tilde{P} u\right\|_{X^{*}}
$$


Here and in the sequel we will omit indices for $W$. After returning to the $(x, t)$ coordinates and taking (1.19) into account this implies Theorem 3 . The reader should note that the choice of $\phi$ depends on both $u$ and $W$. This is essential since for fixed $\phi$ (8.3) cannot hold uniformly for all $u$ and $W$.

Proof. Up to a point the proof follows the steps which were discussed in detail before. We outline the main steps:

STEP 1: Show that the $L^{2}$ Carleman estimate (6.23) holds with $\psi$ replaced by $\Psi$ for all perturbations $k$ as in (8.2). The new conjugated operator $P_{\Psi}$ is obtained from $P_{\psi}$ after conjugating with respect to the weight $e^{k(y, s)}$. This adds a few extra components to the selfadjoint and skewadjoint parts,

$$
\begin{gathered}
L_{\Psi}^{r}=L_{\psi}^{r}+k_{y}^{2}+k_{s}+2 k_{y}\left(\psi_{y}+d\right) \\
L_{\Psi}^{i}=L_{\psi}^{i}-k_{y}(1+d) \partial-\partial k_{y}(1+d)
\end{gathered}
$$

Observing that we can write

$$
k_{y}^{2}+k_{s}+2 k_{y}\left(\psi_{y}+d\right)=\tau d, \quad k_{y}(1+d)=\tau^{\frac{1}{2}} d
$$

with $d$ as in (6.22) we conclude that the conjugated operator $P_{\Psi}$ retains the same form as $P_{\psi}$, therefore the proof of (6.23) rests unchanged.

STEP 2: Show that the symmetric $L^{2}$ Carleman estimate (6.23) holds with $\psi$ replaced by $\Psi$ for all perturbations $k$ as in (8.2). Since $P_{\Psi}$ has the same form as $P_{\psi}$, this argument is identical.

STEP 3: Show that the symmetric mixed $L^{2} \cap L^{p}$ Carleman estimate in Theorem [6 holds with $\psi$ replaced by $\Psi$ for all perturbations $k$ as in (8.2). Since $P_{\Psi}$ has the same form as $P_{\psi}$, this argument is also identical.

STEP 4: Decompose $W$ into a low and a high Hermite-frequency part,

$$
W=W_{\text {low }}+W_{\text {high }}, \quad W_{\text {low }}=\chi_{i}^{1}(x, D) W
$$

where the smooth symbol $\chi_{i}^{1}(x, \xi)$ is supported in $\left\{x^{2}+\xi^{2} \leq 81 \tau\right\}$ and equals 1 in the region $\left\{x^{2}+\xi^{2} \leq 64 \tau\right\}$. Then we show that the high frequency part of $W$ satisfies the desired estimates for all perturbations $k$ as in (8.2), namely

$$
\left\|e^{\Psi} W_{h i g h} \nabla v\right\|_{X^{*}}+\left\|\nabla W_{h i g h} v\right\|_{X^{*}}+\tau^{\frac{1}{2}}\left\|e^{\Psi} W_{h i g h} v\right\|_{X^{*}} \lesssim\left\|e^{\Psi} u\right\|_{X}\|W\|_{L^{n+2}}
$$

After conjugation this becomes

$$
\left\|W_{h i g h} \nabla v\right\|_{X^{*}}+\left\|\nabla W_{h i g h} v\right\|_{X^{*}}+\tau^{\frac{1}{2}}\left\|W_{h i g h} v\right\|_{X^{*}} \lesssim\|v\|_{X}\|W\|_{L^{n+2}}
$$

We only consider the first term on the left. The second one is equivalent by duality, and the third one is similar but simpler. We divide $v$ into two components,

$$
v=\left(1-\chi_{e}^{1}\right) v+\chi_{e}^{1} v
$$

where the smooth symbol $\chi_{e}^{1}(x, \xi)$ is supported in $\left\{x^{2}+\xi^{2} \geq 9 \tau\right\}$ and equals 1 in the region $\left\{x^{2}+\xi^{2} \geq 10 \tau\right\}$. 
For the high frequency component of $v$ we use the $H^{1}$ part of the $X^{2}$ norm to estimate

$$
\left\|W_{h i g h} \nabla \chi_{e}^{1} v\right\|_{L^{\frac{2(n+2)}{n}}} \lesssim\left\|W_{h i g h}\right\|_{L^{n+2}}\left\|\nabla \chi_{e}^{1} v\right\|_{L^{2}} \lesssim\|W\|_{L^{n+2}}\|v\|_{X}
$$

For the low frequency component of $v$ it is still possible to estimate directly the high frequency of the output,

$$
\begin{aligned}
\left\|\chi_{e}^{1}\left(W_{h i g h} \nabla\left(1-\chi_{e}^{1}\right) v\right)\right\|_{H^{-1}} & \lesssim \tau^{-\frac{1}{2}}\left\|W_{h i g h} \nabla\left(1-\chi_{e}^{1}\right) v\right\|_{L^{2}} \\
& \lesssim\left\|W_{h i g h}\right\|_{L^{n+2}} \tau^{-\frac{1}{2}}\left\|\nabla\left(1-\chi_{e}^{1}\right) v\right\|_{L^{\frac{2(n+2)}{n}}} \\
& \lesssim\|W\|_{L^{n+2}}\|v\|_{L^{\frac{2(n+2)}{n}}}
\end{aligned}
$$

Finally, the last remaining part has a much better $L^{2}$ estimate,

$$
\left\|\left(1-\chi_{e}^{1}\right)\left(W_{h i g h} \nabla\left(1-\chi_{e}^{1}\right) v\right)\right\| \lesssim \tau^{-N}\|W\|_{L^{n+2}}\|v\|
$$

which is due to the unbalanced frequency localizations of the two factors.

Due to the estimate (8.4), it suffices to prove (8.3) with $W$ replaced by $W_{\text {low }}$. This allows us to replace the term $\nabla\left(W_{\text {low }} v\right)$ by

$$
\nabla\left(W_{\text {low }} v\right)=W_{\text {low }} \nabla v+\left(\nabla W_{\text {low }}\right) v
$$

where we can estimate

$$
\left\|\nabla W_{\text {low }}\right\|_{L^{n+2}} \lesssim \tau^{\frac{1}{2}}\|W\|_{L^{n+2}}
$$

Hence without any restriction in generality we can drop the third term in (8.3) and show that we can choose the perturbation $k$ so that

$$
\left\|e^{\Psi} W \nabla u\right\|_{X^{*}}+\tau^{\frac{1}{2}}\left\|e^{\Psi} W u\right\|_{X^{*}} \lesssim\left\|e^{\Psi(x)} \tilde{P} u\right\|_{X^{*}}
$$

STEP 5: Show that, given $u$ and $W$, we can choose the perturbation $k$ so that (8.5) holds. At this stage we no longer need the full $X^{*}$ norm for the $W$ terms, it suffices instead to consider the $L^{\frac{2(n+2)}{n+4}}$ norm. Begin with the unperturbed integral

$$
\int_{\mathbb{R}} \int_{\mathbb{R}}^{n} F_{\psi} d x d t, \quad F_{\psi}=\left|e^{\psi} W \nabla u\right|^{\frac{2(n+2)}{n+4}}+\left|\tau^{\frac{1}{2}} e^{\psi} W u\right|^{\frac{2(n+2)}{n+4}}
$$

We can select a subset $I$ of $\mathbb{R}$ consisting of time intervals of length 1 with unit separation at least 8 so that

$$
\int_{\mathbb{R}} \int_{\mathbb{R}}^{n} F_{\psi} d x d t \lesssim \int_{I} \int_{\mathbb{R}}^{n} F_{\psi} d x d t
$$

By a small abuse of notation we label

$$
I=\bigcup_{i \in \mathcal{I}} I_{i}, \quad I_{i} \subset[i-1, i+1]
$$


We define a family of perturbations $k$ depending on parameters $b_{i}, \sigma_{i}$ by

$$
\begin{gathered}
k(y, s)=\sum_{i \in \mathcal{I}} \varepsilon_{i}\left(100 \chi_{3 \tau I_{i}}+\chi_{2 I_{i}} \chi_{|y|^{2} \leq \tau}\left(b_{i} y+\sigma_{i}(s-i)\right)\right), \\
\left|b_{i}\right| \leq \tau^{\frac{1}{2}}, \quad\left|\sigma_{i}\right| \leq \tau
\end{gathered}
$$

Due to the choice of the intervals $I_{i}$ it is easy to see that after changing the weight $\psi$ to $\Psi$ we retain the concentration to a dilate of $I$,

$$
\int_{\mathbb{R}} \int_{\mathbb{R}^{n}} F_{\Psi} d x d t \lesssim \int_{3 I} \int_{\mathbb{R}^{n}} F_{\Psi} d x d t
$$

The choice of the parameters $b_{i}, \sigma_{i}$ can be made independently for each $i$. We consider two cases.

i) Suppose $\varepsilon_{i} \lesssim \tau^{-\frac{1}{2}}$. Then the choice of the parameters is irrelevant since in $3 I_{i}$ we can estimate

$$
\begin{aligned}
\left\|e^{\psi} W \nabla u\right\|_{L^{\frac{2(n+2)}{n+4}}}+\tau^{\frac{1}{2}}\left\|e^{\psi} W u\right\|_{L^{\frac{2(n+2)}{n+4}}} & \lesssim\|W\|_{L^{n+2}}\left(\left\|e^{\Psi} \nabla u\right\|_{L^{2}}+\tau^{\frac{1}{2}}\left\|e^{\Psi} u\right\|_{L^{2}}\right) \\
& \lesssim\left(\tau^{-1 / 2}\left\|e^{\Psi} \nabla u\right\|_{L^{2}}+\left\|e^{\Psi} u\right\|_{L^{2}}\right) \\
& \lesssim\|u\|_{X_{2}}
\end{aligned}
$$

ii) Suppose $\varepsilon_{i} \gg \tau^{-\frac{1}{2}}$. Then we need to choose the parameters $b_{i}, \sigma_{i}$ in a favorable manner. This choice is made using Wolff's Lemma:

Lemma 8.1 (Wolff's Lemma 29]). Let $\mu$ be a measure in $\mathbb{R}^{n}$ and $B$ a convex set. Then one can find $b_{k} \in B$ and disjoint convex sets $E_{k} \subset \mathbb{R}^{n}$ so that the measures $e^{x b_{k}} \mu$ are concentrated in $E_{k}$,

$$
\int_{E_{k}} e^{x b_{k}} d \mu \gtrsim \frac{1}{2} \int_{E_{k}} e^{x b_{k}} d \mu
$$

and

$$
\sum\left|E_{k}\right|^{-1} \gtrsim|B|
$$

We apply the lemma for the measures

$$
d \mu_{i}=1_{3 I_{i}} F_{\psi}
$$

In our case we have

$$
B_{i}=\delta \varepsilon_{i}\left([-\tau, \tau] \times B\left(0, \tau^{\frac{1}{2}}\right)\right), \quad\left|B_{i}\right| \approx \varepsilon_{i}^{n+1} \tau^{\frac{n+2}{2}}
$$

Hence we can find parameters $b_{i}^{k}$ and $\sigma_{i}^{k}$ and convex sets $E_{i}^{k} \subset 3 I_{i} \times B\left(0,3 \tau^{\frac{1}{2}}\right)$ so that the corresponding measures $F_{\Psi_{k}}$ are concentrated in $E_{i}^{k}$ with

$$
\sum\left|E_{i}^{k}\right|^{-1} \gtrsim \varepsilon_{i}^{n+1} \tau^{\frac{n+2}{2}}
$$

At the same time we have

$$
\sum \int_{E_{i}^{k}}|W|^{n+2} d x d t \lesssim \varepsilon_{i}^{n+2}
$$


Hence we can choose $k$ so that

$$
\int_{E_{i}^{k}}|W|^{n+2} d x d t \lesssim \varepsilon_{i} \tau^{-\frac{n+2}{2}}\left|E_{i}^{k}\right|^{-1}
$$

which by Holder's inequality leads to

$$
\|W\|_{L^{\frac{n+2}{2}}\left(E_{i}^{k}\right)} \lesssim \varepsilon_{i}^{\frac{1}{n+2}} \tau^{-\frac{1}{2}}
$$

Denoting this index $k$ by $k(i)$ we can write

$$
\begin{aligned}
\left\|e^{\Psi} W\left(\nabla, \tau^{\frac{1}{2}}\right) u\right\|_{l^{2} L^{\frac{2(n+2)}{n+4}}} & \lesssim\left\|e^{\Psi} W\left(\nabla, \tau^{\frac{1}{2}}\right) u\right\|_{l_{i}^{2} L^{\frac{2(n+2)}{n+4}}\left(I_{i}\right)} \\
& \lesssim\left\|e^{\Psi} W\left(\nabla, \tau^{\frac{1}{2}}\right) u\right\|_{l_{i}^{2} L^{\frac{2(n+2)}{n+4}}\left(E_{i}^{k(i)}\right)} \\
& \lesssim\left\|W\left(\nabla, \tau^{\frac{1}{2}}\right)\left(e^{\Psi} u\right)\right\|_{l_{i}^{2} L^{\frac{2(n+2)}{n+4}}\left(E_{i}^{k(i)}\right)}
\end{aligned}
$$

Decomposing the function $v=e^{\Psi} u$ into low and high frequencies we further estimate

$$
\begin{aligned}
\left\|e^{\Psi} W\left(\nabla, \tau^{\frac{1}{2}}\right) u\right\|_{l^{2} L^{\frac{2(n+2)}{n+4}} \lesssim} & \left\|W\left(\nabla, \tau^{\frac{1}{2}}\right)\left(1-\chi_{i}^{1}(x, D)\right)\left(e^{\Psi} u\right)\right\|_{l_{i}^{2} L^{\frac{2(n+2)}{n+4}}\left(I_{i}\right)} \\
& +\left\|W\left(\nabla, \tau^{\frac{1}{2}}\right) \chi_{i}^{1}(x, D)\left(e^{\Psi} u\right)\right\|_{l_{i}^{2} L^{\frac{2(n+2)}{n+4}}\left(E_{i}^{k(i)}\right)}
\end{aligned}
$$

The first term on the right is estimated as in Step 4,

$$
\begin{aligned}
&\left\|W\left(\nabla, \tau^{\frac{1}{2}}\right)\left(1-\chi_{i}^{1}(x, D)\right)\left(e^{\Psi} u\right)\right\|_{l_{i}^{2} L^{\frac{2(n+2)}{n+4}}\left(I_{i}\right)} \\
& \lesssim\|W\|_{l^{\infty} L^{n+2}}\left\|\left(1-\chi_{i}^{1}(x, D)\right)\left(e^{\Psi} u\right)\right\|_{H^{1}} \\
& \lesssim\|W\|_{l^{\infty} L^{n+2}}\left\|e^{\Psi} u\right\|_{X}
\end{aligned}
$$

It is only for the second term on the right that we need to use (8.6):

$$
\begin{aligned}
& \left\|W\left(\nabla, \tau^{\frac{1}{2}}\right) \chi_{i}^{1}(x, D)\left(e^{\Psi} u\right)\right\|_{l_{i}^{2} L^{\frac{2(n+2)}{n+4}}\left(E_{i}^{k(i)}\right)} \\
& \lesssim\|W\|_{l_{i}^{\infty} L^{\frac{n+2}{2}}\left(E_{i}^{k(i)}\right)}\left\|\left(\nabla, \tau^{\frac{1}{2}}\right) \chi_{i}^{1}(x, D)\left(e^{\Psi} u\right)\right\|_{l_{i}^{2} L^{\frac{2(n+2)}{n+4}}\left(E_{i}^{k(i)}\right)} \\
& \lesssim\left\|e^{\Psi} u\right\|_{l^{2} L^{\frac{2(n+2)}{n}}} \\
& \lesssim\left\|e^{\Psi} u\right\|_{X}
\end{aligned}
$$

The proof of the Theorem is concluded.

\section{Appendix A. The change of coordinates}

Suppose that the coefficients $g$ satisfy (1.15). In this section we verify that we can change coordinates so that (1.15) and (1.16) are both satisfied. Due to the anisotropic character of the equation we must leave the time variable unchanged and consider changes of coordinates which have the form

$$
(t, x) \rightarrow(s, y), \quad s=t, y=\chi(t, x) .
$$


The expression for the operator $P$ in the new coordinates is

$$
P=\partial_{t}+\partial_{k} \tilde{g}^{k l}(t, y) \partial_{l}+\frac{x_{k}}{t} \tilde{d}^{k l} \partial_{l}
$$

where the new coefficients $\tilde{g}, \tilde{d}$ are computed using the chain rule,

$$
\tilde{g}^{k l}=\frac{\partial \chi_{l}}{\partial x_{i}} g^{i j} \frac{\partial \chi_{k}}{\partial x_{j}}, \quad \frac{x_{k}}{t} \tilde{d}^{k l}=\frac{\partial \chi_{l}}{\partial t}-\left[D \chi_{l m}^{-1}\left(\partial_{x_{m} x_{i}}^{2} \chi_{l}\right)\right] g^{i j} \frac{d \chi_{l}}{\partial x_{j}}
$$

There is a price to pay for this, namely in the new coordinates we obtain lower order terms which cannot be treated perturbatively. Instead we obtain coefficients $\tilde{d}^{k}$ which have the same regularity and size as $g_{1}-I_{n}$.

The Lipschitz condition (1.15) ensures that $g$ has a limit at $(0,0)$ so we assume that $g$ is continuous. After a linear change of coordinates we may and do choose $g$ with $g(0,0)=I_{n}$. Again by (1.15) this implies

$$
\left|g(t, x)-I_{n}\right| \ll 1
$$

Proposition A.1. Let $g$ be a metric which satisfies (1.15) with $g(0,0)=I_{n}$. Then there is change of coordinates $(t, y)=(t, \chi(t, x))$ which is close to the identity

$$
\left\|\partial_{x} \chi-I_{n}\right\|_{L^{\infty}} \ll 1
$$

and has regularity

$$
\sup _{\tau}\left\|\left(t+x^{2}\right)^{-1 / 2}\left(t \partial_{t}\right)^{\alpha}\left(\left(t+x^{2}\right)^{1 / 2} \partial_{x}\right)^{\beta} \chi\right\|_{l^{1}\left(A(\tau) ; L^{\infty}\right)} \ll 1, \quad 2 \leq 2 \alpha+|\beta| \leq 4
$$

so that in the new coordinates both functions $\tilde{g}$ and $\tilde{d}$ satisfy (1.15), while $\tilde{g}-I_{n}$ and $\tilde{d}$ satisfy (1.16).

Proof. Consider the covering of the $[0,2] \times B(0,2)=\cup A_{i j}$ with an associated smooth partition of unity $\eta_{i j}$. We can assume that the functions $\eta_{i j}$ satisfy

$$
\left|\partial_{t}^{\alpha} \partial_{x}^{\beta} \eta_{i j}\right| \lesssim c_{\alpha \beta} t^{-\alpha}\left(t+x^{2}\right)^{-\frac{\beta}{2}}
$$

We choose the points

$$
\left(t_{i}, x_{i j}\right)=\left(e^{-4 i}, e^{-2 i+j}\right) \in A_{i j} .
$$

and insure that $\eta_{i j}=1$ near $\left(t_{i}, x_{i j}\right)$. By (1.15) we have

$$
\sup _{\tau} \sum_{(i, j) \in \mathcal{A}(\tau)}\left|g\left(t_{i}, x_{i j}\right)-g\left(t_{i}, x_{i,(j-1)}\right)\right|+\left|g\left(t_{i}, x_{i j}\right)-g\left(t_{i+1}, x_{(i+1), j}\right)\right| \ll 1 .
$$

Within a fixed set $A_{i j}$ we consider the linear map defined by the matrix

$$
\chi_{i j}=g^{-1 / 2}\left(t_{i}, x_{i j}\right) \text {. }
$$

It transforms the coefficients at $\left(t_{i}, x_{i j}\right)$ to the identity and has the desired properties within $A_{i j}$. We assemble the maps defined by $\chi_{i j}$ using the partition of unity,

$$
\chi(t, x)=\sum_{55} \eta_{i j}(t, x) \chi_{i j} x
$$


Then

$$
\nabla \chi(t, x)-I_{n}=\sum\left(\nabla \eta_{i j}\right) \chi_{i j} x+\eta_{i j}\left(\chi_{i j}-I_{n}\right),
$$

Let $(t, x) \in A_{i_{0}, j_{0}}$. Since $\sum \nabla \eta_{i j}=0$ we have

$$
\nabla_{x} \chi(t, x)-I_{n}=\sum_{\eta_{i j}(t, x)>0} \nabla_{x} \eta_{i j}(t, x)\left(\chi_{i j}-\chi_{i_{0}, j_{0}}\right)+\eta_{i j}\left(\chi_{i j}-I_{n}\right) .
$$

The first term on the right hand is small by (A.5) (for $\chi_{i j}$ ) and the second one by (A.1) therefore the smallness of $\nabla \chi-I_{n}$ follows.

For the second order spatial derivatives we write

$$
\begin{aligned}
D_{x}^{2} \chi(t, x) & =\sum D^{2} \eta_{i j}(t, x) \chi_{i j} x+2 D_{x} \eta_{i j}(t, x) \chi_{i j} \\
& =\sum D_{x}^{2} \eta_{i j}(t, x)\left(\chi_{i j}-\chi_{i_{0}, j_{0}}\right) x+2 D_{x} \eta_{i j}\left(\chi_{i j}-\chi_{i_{0}, j_{0}}\right) .
\end{aligned}
$$

Hence by (A.4) and (A.5) (again for $\chi_{i j}$ ) we obtain

$$
\left\|\left(|x|^{2}+t\right)^{1 / 2} D_{x}^{2} \chi\right\|_{l^{1}\left(A(\tau) ; L^{\infty}\right)} \ll 1 .
$$

Also

$$
\partial_{t} \chi(t, x)=\sum \partial_{t} \eta_{i j}\left(\chi_{i j}-\chi_{i_{0} j_{0}}\right) x
$$

gives the desired bound for the time derivative. A similar computation yields the bound for the higher order derivatives in (A.3).

Consider now the new metric $\tilde{g}$. Since both $D \chi$ and $(D \chi)^{-1}$ are Lipschitz on the dyadic scale with $l^{1}(A(\tau))$ summability, from (1.15) for $g$ we easily obtain (1.15) for $\tilde{g}$. In addition, our construction insures that $\tilde{g}\left(t_{i}, x_{i j}\right)=I_{n}$. This in turn leads to the bound

$$
\left\|\tilde{g}-I_{n}\right\|_{L^{\infty}\left(A_{i j}\right)} \lesssim\|\tilde{g}\|_{\operatorname{Lip}_{x}\left(A_{i j}\right)}+\|\tilde{g}\|_{C_{t}^{m_{i j}}\left(A_{i j}\right)}
$$

which shows that (1.15) for $\tilde{g}$ implies (1.16) for $\tilde{g}-I_{n}$.

It remains to consider the lower order terms. From $\partial_{t} \chi$ we obtain coefficients $\tilde{d}$ of the form

Within $A_{i_{0}, j_{0}}$ this gives

$$
\tilde{d}=t \sum \partial_{t} \eta_{i j} \chi_{i j}
$$

$$
\tilde{d}=t \sum \partial_{t} \eta_{i j}(t, x)\left(\chi_{i j}-\chi_{i_{0} j_{0}}\right)
$$

The functions $t \partial_{t} \eta_{i j}(t, x)$ are bounded and smooth on the dyadic scale, while the $l^{1}(A(\tau))$ summability comes from the $\chi_{i j}-\chi_{i_{0} j_{0}}$ factor due to (A.5). Hence both (1.15) and (1.16) are satisfied.

The contribution of $\partial_{x}^{2} \chi$ to $\tilde{d}$ has the form

$$
\tilde{d}=\frac{x t}{x^{2}}\left(\partial_{x} \chi\right)^{-1}\left(\partial_{x}^{2} \chi\right) g
$$

There is no singularity at $x=0$ since $\chi$ is linear in $x$ for $x^{2} \ll t$. Then from (A.2) and (A.3) we obtain

$$
|\tilde{d}| \lesssim \frac{t}{x^{2}}
$$


with added $l^{1}(A(\tau))$ summability inherited from $\partial_{x}^{2} \chi$. This is better than (1.16), and in effect this term can be included in $W$ and treated perturbatively. The bound (1.16) is also easy to obtain from the similar bounds for $g$ and derivatives of $\chi$.

\section{REFERENCES}

[1] Giovanni Alessandrini and Sergio Vessella. Local behaviour of solutions to parabolic equations. Comm. Partial Differential Equations, 13(9):1041-1058, 1988.

[2] Giovanni Alessandrini and Sergio Vessella. Remark on the strong unique continuation property for parabolic operators. Proc. Amer. Math. Soc., 132(2):499-501 (electronic), 2004.

[3] N. Aronszajn. A unique continuation theorem for solutions of elliptic partial differential equations or inequalities of second order. J. Math. Pures Appl. (9), 36:235-249, 1957.

[4] N. Aronszajn, A. Krzywicki, and J. Szarski. A unique continuation theorem for exterior differential forms on Riemannian manifolds. Ark. Mat., 4:417-453 (1962), 1962.

[5] T. Carleman. Sur un problème d'unicité pur les systèmes d'équations aux dérivées partielles à deux variables indépendantes. Ark. Mat., Astr. Fys., 26(17):9, 1939.

[6] Xu-Yan Chen. A strong unique continuation theorem for parabolic equations. Math. Ann., 311(4):603-630, 1998.

[7] L. Escauriaza, F. J. Fernández, and S. Vessella. Doubling properties of caloric functions. Appl. Anal., 85(1-3):205-223, 2006.

[8] Luis Escauriaza. Carleman inequalities and the heat operator. Duke Math. J., 104(1):113-127, 2000.

[9] Luis Escauriaza and Francisco Javier Fernández. Unique continuation for parabolic operators. Ark. Mat., 41(1):35-60, 2003.

[10] Luis Escauriaza and Luis Vega. Carleman inequalities and the heat operator. II. Indiana Univ. Math. J., 50(3):1149-1169, 2001.

[11] F. J. Fernandez. Unique continuation for parabolic operators. II. Comm. Partial Differential Equations, 28(9-10):1597-1604, 2003.

[12] Lars Hörmander. Uniqueness theorems for second order elliptic differential equations. Comm. Partial Differential Equations, 8(1):21-64, 1983.

[13] Lars Hörmander. The analysis of linear partial differential operators. IV, volume 275 of Grundlehren der Mathematischen Wissenschaften [Fundamental Principles of Mathematical Sciences]. Springer-Verlag, Berlin, 1994. Fourier integral operators, Corrected reprint of the 1985 original.

[14] David Jerison. Carleman inequalities for the Dirac and Laplace operators and unique continuation. Adv. in Math., 62(2):118-134, 1986.

[15] David Jerison and Carlos E. Kenig. Unique continuation and absence of positive eigenvalues for Schrödinger operators. Ann. of Math. (2), 121(3):463-494, 1985. With an appendix by E. M. Stein.

[16] G. E. Karadzhov. Riesz summability of multiple Hermite series in $L^{p}$ spaces. $C . R$. Acad. Bulgare Sci., 47(2):5-8, 1994.

[17] Herbert Koch and Daniel Tataru. Carleman estimates and unique continuation for second-order elliptic equations with nonsmooth coefficients. Comm. Pure Appl. Math., 54(3):339-360, 2001.

[18] Herbert Koch and Daniel Tataru. Dispersive estimates for principally normal pseudodifferential operators. Comm. Pure Appl. Math., 58(2):217-284, 2005.

[19] Herbert Koch and Daniel Tataru. $L^{p}$ eigenfunction bounds for the Hermite operator. Duke Math. J., 128(2):369-392, 2005. 
[20] Fang-Hua Lin. A uniqueness theorem for parabolic equations. Comm. Pure Appl. Math., 43(1):127-136, 1990.

[21] Sigeru Mizohata. Unicité du prolongement des solutions pour quelques opérateurs différentiels paraboliques. Mem. Coll. Sci. Univ. Kyoto. Ser. A. Math., 31:219-239, 1958.

[22] Chi-Cheung Poon. Unique continuation for parabolic equations. Comm. Partial Differential Equations, 21(3-4):521-539, 1996.

[23] Jean-Claude Saut and Bruno Scheurer. Unique continuation for some evolution equations. J. Differential Equations, 66(1):118-139, 1987.

[24] C. D. Sogge. A unique continuation theorem for second order parabolic differential operators. Ark. Mat., 28(1):159-182, 1990.

[25] Christopher D. Sogge. Concerning the $L^{p}$ norm of spectral clusters for second-order elliptic operators on compact manifolds. J. Funct. Anal., 77(1):123-138, 1988.

[26] Christopher D. Sogge. Oscillatory integrals and unique continuation for second order elliptic differential equations. J. Amer. Math. Soc., 2(3):491-515, 1989.

[27] Christopher D. Sogge. Strong uniqueness theorems for second order elliptic differential equations. Amer. J. Math., 112(6):943-984, 1990.

[28] S. Thangavelu. Summability of Hermite expansions. I, II. Trans. Amer. Math. Soc., 314(1):119-142, 143-170, 1989.

[29] T.H. Wolff. A property of measures in $\mathbf{R}^{\mathbf{N}}$ and an application to unique continuation. Geom. Funct. Anal., 2(2):225-284, 1992.

[30] Hidehiko Yamabe. A unique continuation theorem of a diffusion equation. Ann. of Math. (2), 69:462-466, 1959.

Mathematisches Institut der Universität Bonn, Beringstr.1, 53115 Bonn, GERMANY

E-mail address: koch@math.uni-bonn.de

Department of Mathematics, University of California, Berkeley, CA 94720

E-mail address: tataru@math.berkeley.edu 